\title{
Engineering Design for the Magnetic Diagnostics of Wendelstein 7-X
}

M. Endler*, B. Brucker, V. Bykov, A. Cardella, A. Carls, F. Dobmeier ${ }^{1}$,

A. Dudek, J. Fellinger, J. Geiger, K. Grosser, O. Grulke, D. Hartmann, D. Hathiramani, K. Höchel, M. Köppen, R. Laube, U. Neuner, X. Peng ${ }^{2}$, K. Rahbarnia, K. Rummel, T. Sieber, S. Thiel, A. Vorköper, A. Werner, T. Windisch, M. Y. Ye ${ }^{3}$

Max-Planck-Institut für Plasmaphysik, D-17491 Greifswald

${ }^{1}$ Fakultät für Maschinenwesen, Technische Universität München, D-85747 Garching

2present address: Institute of Plasma Physics, Chinese Academy of

Sciences, P.O. Box 1126, 230031, Hefei Anhui, P.R. China

${ }^{3}$ present address: School of Nuclear Science and Technology, University of Science and Technology of China, 230026, Hefei Anhui, P.R. China

25th August 2015

\begin{abstract}
The magnetic diagnostics foreseen for the Wendelstein 7-X (W7-X) stellarator are diamagnetic loops to measure the plasma energy, Rogowski coils to measure the toroidal plasma current, saddle coils to measure the Pfirsch-Schlüter currents, segmented Rogowski coils (poloidal magnetic field probes) to add information on the distribution of the plasma current density, and Mirnov coils to observe magnetohydrodynamic modes. All these magnetic field sensors were designed as classical pick-up coils, after the time integration of induced signals for $1 / 2$ hour had been successfully demonstrated.

The long-pulse operation planned for W7-X causes nevertheless significant challenges to the design of these diagnostics, in particular for the components located inside the plasma vessel, which may be exposed to high levels of microwave (electron cyclotron resonance) stray radiation and thermal radiation. This article focuses on the tests and modelling performed during the development of the magnetic diagnostics and on the design solutions adopted to meet the conflicting requirements.
\end{abstract}

*Corresponding author, email: endler@ipp.mpg.de 
All pick-up coils foreseen for the initial operation phase of W7-X and their signal cable sections inside the plasma vessel and the cryostat are now installed, and their electronics and data acquisition are under preparation.

\section{Keywords}

Stellarator; Wendelstein 7-X; Magnetic diagnostics

\section{Contents}

1 Introduction 3

2 Purpose, location and distribution of magnetic sensors 5

2.1 Physics criteria . . . . . . . . . . . . . . . 5

2.1 .1 General ...................... 5

2.1 .2 Diamagnetic loops . . . . . . . . . . . . . . . 6

2.1.3 Saddle coils . . . . . . . . . . . . . . . . . . . . . 8

2.1.4 Rogowski coils (including segmented Rogowski coils) . . . . . . . . 10

2.1.5 Mirnov coils . . . . . . . . . . . . . . . . . . 12

2.2 Location inside or outside the plasma vessel . . . . . . . . . . . . . . . . . 13

2.3 Redundancy . . . . . . . . . . . . . . . . . . . . . . . 15

2.4 Asymmetries . . . . . . . . . . . . . . . . . . . . . 15

3 Major challenges $\quad 16$

3.1 Long-pulse integration . . . . . . . . . . . . . . . . . 16

3.1.1 Offset voltages generated within the electronics . . . . . . . . . 16

3.1 .2 Thermovoltages . . . . . . . . . . . . . . . 16

3.1.2.1 Magnitude ................... 17

3.1.2.2 Reducing thermovoltages in plug connectors . . . . . . . . 17

3.1.3 Dynamic range . . . . . . . . . . . . . . . . . . . 18

3.1.4 Further sources of error in long-pulse integration . . . . . . . . . . 19

3.2 Microwave stray radiation . . . . . . . . . . . . . . . . . . . . . . 19

3.3 Additional thermal load by thermal radiation . . . . . . . . . . . . . 22

3.4 Design principles to meet the challenges . . . . . . . . . . . . . 22

4 Diamagnetic loops $\quad 24$

4.1 Design and testing ......................... 24

4.1.1 Winding and basic design . . . . . . . . . . . . . . . 24

4.1.2 Choice of materials and time resolution . . . . . . . . . . . . 26

4.1.3 Dimensioning and thermal balance of final design . . . . . . . . . . 29

4.1.3.1 Flat sections . . . . . . . . . . . . . . . . . . 29

4.1.3.2 Sections with compensation coils . . . . . . . . . . . 29

4.1 .4 Electromagnetic forces . . . . . . . . . . . . . . . . . . 34

4.1.4.1 Flat sections . . . . . . . . . . . . . . . . 34

4.1.4.2 Sections with compensation coils . . . . . . . . . 35 
4.2 Expected signals . . . . . . . . . . . . . . . . . . 35

5 Saddle coils $\quad 37$

5.1 Design . . . . . . . . . . . . . . . . . . . . 37

5.2 Expected signals . . . . . . . . . . . . . . . . . . . 37

6 Rogowski coils (including segmented Rogowski coils) 39

6.1 Design and testing . . . . . . . . . . . . . . . . . 39

6.1.1 Winding and basic design . . . . . . . . . . . . . . . 39

6.1.2 Choice of materials and time resolution . . . . . . . . . . . . . 43

6.1.3 Dimensioning and thermal balance of final design for in-vessel Rogowski coils . . . . . . . . . . . . . . . . . 43

6.1.4 Electromagnetic forces . . . . . . . . . . . . . . . . . . 48

6.2 Expected signals . . . . . . . . . . . . . . . . . . . . . . . 49

7 Mirnov coils $\quad 50$

7.1 Design and testing . . . . . . . . . . . . . . 50

7.1 .1 Basic design . . . . . . . . . . . . . . . . 5 50

7.1.1.1 Coils behind gaps between wall protection tiles . . . . . . 51

7.1.1.2 Coils on the plasma side of wall protection panels . . . . . 52

7.1.2 Time resolution . . . . . . . . . . . . . . . . 53

7.1.3 Thermal balance and choice of materials . . . . . . . . . . . . 54

7.2 Expected signals . . . . . . . . . . . . . . . . . 56

8 Signal cables $\quad 58$

8.1 Type of cable . . . . . . . . . . . . . . . . . . . 58

8.2 ECR stray radiation shielding . . . . . . . . . . . . . . . . . 58

8.3 Cabling outside the vacuum barriers . . . . . . . . . . . . . . . 60

9 Electronics and data acquisition for the magnetic equilibrium diagnos$\begin{array}{ll}\text { tics } & 60\end{array}$

10 Conclusion and outlook $\quad 61$

$\begin{array}{ll}\text { Acknowledgements } & 63\end{array}$

$\begin{array}{ll}\text { References } & 63\end{array}$

\section{Introduction}

Wendelstein 7-X (W7-X) is a large modular stellarator with superconducting field coils, presently under commissioning at the Max-Planck-Institut für Plasmaphysik in Greifswald, Germany [1,2]. The purpose of the magnetic diagnostics is the determination of the plasma energy, the measurement of the toroidal plasma current, the support of the plasma equilibrium reconstruction, and the observation of magnetohydrodynamic (MHD) modes. For these tasks, a number of pick-up coils of different types is usually distributed 
inside and outside the plasma vessel (PV) of stellarators and tokamaks. The first three tasks are then accomplished by integrating the voltages induced in appropriately arranged pick-up coils from the beginning of a plasma discharge (i. e., from a state with zero plasma currents). This set of pick-up coils is called the magnetic equilibrium diagnostics (MEDs). The measurement of magnetic field variations due to MHD modes requires small pick-up coils with low inductance, located close to the plasma, which are called Mirnov coils.

The plan to operate W7-X in plasma discharges of up to $1800 \mathrm{~s}$ with considerable heating power results in several challenges to the design of these diagnostics (for a general discussion of diagnostics for long-pulse discharges, see [3]). Although none of the magnetic sensors finally realised is novel in its nature, the long-pulse challenge in highpower discharges resulted in specific design solutions. It is the purpose of this article to communicate these solutions and the underlying considerations and tests.

After it had been demonstrated that an integrator could be built with sufficiently low drift for half-hour discharges [4], the decision was taken to use, for the time being, traditional pick-up coils as magnetic sensors in W7-X. Specifically, the aim of the development of MEDs for W7-X was

1. to measure the diamagnetic energy with a total error below $10 \mathrm{~kJ}$ (from the diamagnetic energy and the heating power, or from the time evolution of the diamagnetic energy after changing the heating power, the energy confinement time can be calculated). The maximum expected value of the diamagnetic energy is $5 \mathrm{MJ}$ under largest envisaged heating power and good energy confinement;

2. to measure the toroidal plasma current with a total error below $100 \mathrm{~A}$ (at a maximum expected value below $200 \mathrm{kA}$ for electron cyclotron or neutral beam current drive). The high accuracy of the plasma current measurement is desired, because a low toroidal net current (bootstrap current) is one of the optimisation criteria of W7-X $[5]$

3. to obtain information about the distribution of the toroidal current density (PfirschSchlüter (PS) currents and higher moments of the distribution).

Diamagnetic loops (DLs), Rogowski coils (RCs) and saddle coils (SCs) are used to obtain these quantities. A basic description of the different coil types and their functionality is given in section 2.1. For a more detailed treatment see, e.g., [6]. A discussion of the use of the different W7-X MEDs for the reconstruction of the quantities characterising a plasma configuration can be found in [7].

At a later stage, the use of magnetic sensors other than pick-up coils may be reconsidered, like, e.g., fiber optic based current sensors for a non-integrated measurement of the plasma current [8]. Furthermore, additional Mirnov coils for magnetic fluctuation measurements will probably be used in the heads of probe manipulators penetrating into the scrape-off layer or edge plasma. Those supplementary W7-X magnetic diagnostics will not be treated in this article.

We describe the general layout of the W7-X magnetic diagnostics in section 2 and list the major challenges they will have to tackle in section 3 . In the subsequent sections, we specifically address the design, test measurements and numerical modelling of the DLs 
(section 4), SCs (section 5), RCs (section 6) and Mirnov coils (section 7). Section 8 is dedicated to the signal cables. The electronics and data acquisition are described in section 9, and we conclude in section 10. A list of abbreviations not in common use is supplied at the end of the article.

\section{Purpose, location and distribution of magnetic sen- sors}

\subsection{Physics criteria}

\subsubsection{General}

In contrast to a tokamak, in a stellarator different toroidal positions are not physically equivalent. In W7-X, there remains in theory a ten-fold symmetry of the plasma configuration: The entire system can be created from one "half module" (HM) by first rotating the half module by $180^{\circ}$ around a radial axis through one of its end planes, creating one module, and then multiplying this module by repeatedly shifting it by $72^{\circ}$ in toroidal direction, until the entire torus of five modules is complete. The entire magnetic information for the reconstruction of the plasma equilibrium could therefore be obtained from measurements in one single half module.

Within one half module, the plasma cross section varies from bean-shaped elongated flux surfaces ("bean-shaped plane", BP) to triangular-shaped ones ("triangular plane", TP) [9, fig. 3]. These two planes exhibit a maximum contrast in view of

- magnetic curvature and radial magnetic field gradient (which is highest in BPs and almost 0 in TPs),

- magnetic field strength in mirror configurations (and hence ratio between trapped and passing particles — plus the option to trap the particles in either the BPs or the TPs), and

- multipolarity of the poloidal magnetic field generated by a toroidal plasma current.

The latter contrast will even allow to obtain a limited amount of information on the radial distribution of the plasma current by comparing the signals from segmented Rogowski coils (SRCs, see section 2.1.4) between BP and TP.

It should be noted that the sensors are not located exactly in the BPs or TPs, but shifted by several tens of centimetres into the HM (toroidal length of one HM: $\sim 3 \mathrm{~m}$ ), depending on the available space between PV and wall protection elements (WPEs) and due to the positions of ports and further components.

The set of MEDs comprises DLs in the BP and in the TP (see section 2.1.2), SCs over a short section of the half module close to the TP and over the longer remaining section up to the BP (see section 2.1.3), continuous Rogowski coils (CRCs) only in the TP (there is no appropriate continuous space near the BP), and SRCs in the $\mathrm{TP}$ and in the $\mathrm{BP}$ (although several segments from the BP had to be shifted toroidally out of the BP; see section 2.1.4). The location of the Mirnov coils is treated in section 2.1.5. In fig. 1 and 


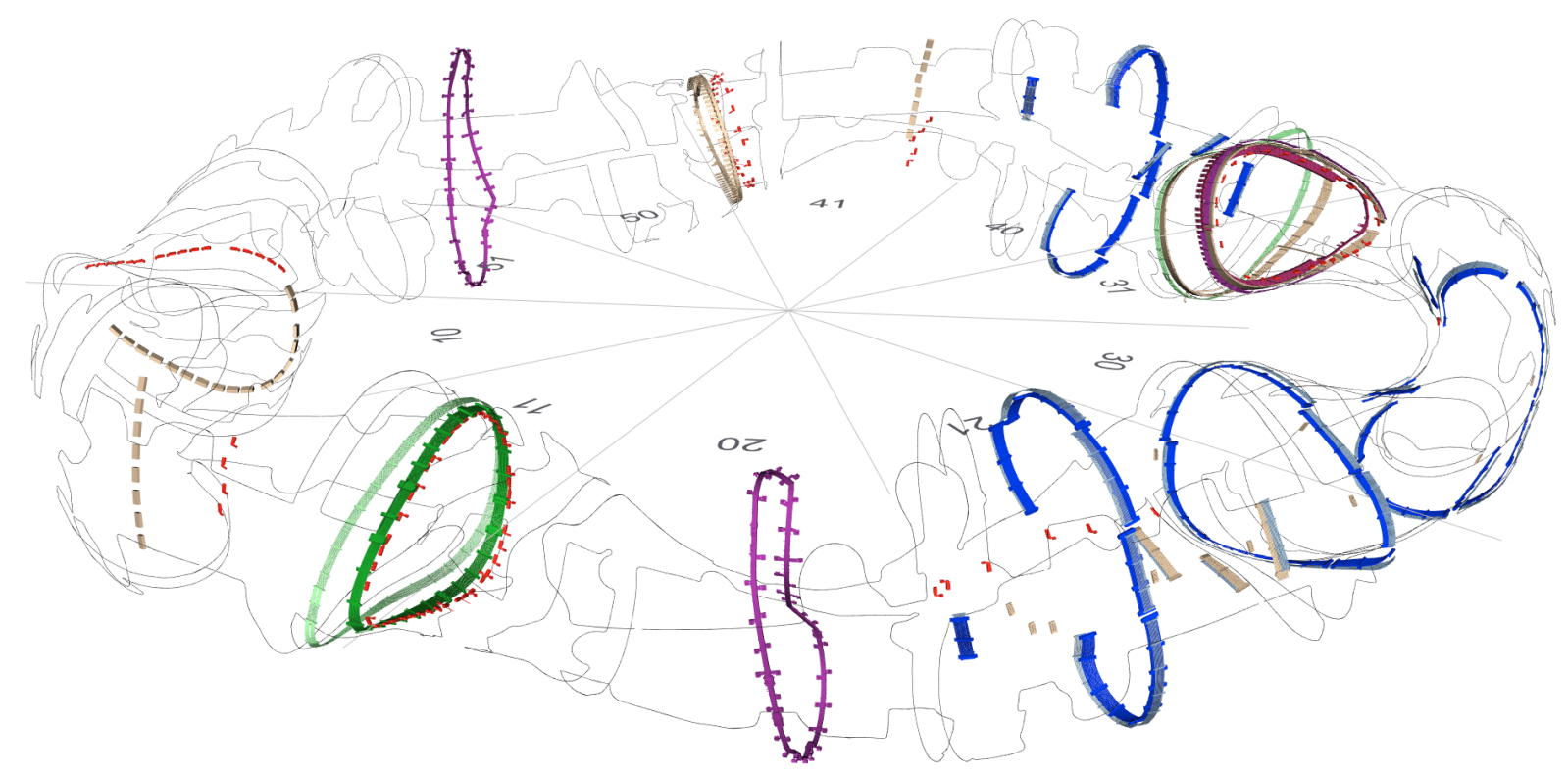

Figure 1: Overview of magnetic diagnostics in W7-X: The numbers indicate ten different half modules of the device. The saddle coils are shown in black, the continuous Rogowski coils in green (saturated for in-vessel, light for outside the plasma vessel), the segmented Rogowski coils in blue (saturated for in-vessel, light for outside the plasma vessel), the diamagnetic loops in violet, and the Mirnov coils in red. Beige colour indicates that this coil will be installed later (compare table 1).

table 1, an overview on the location of the W7-X magnetic sensors is given. Several of these sensors will not be installed before the first, short-pulse, operation phase of W7-X [10].

\subsubsection{Diamagnetic loops}

The DLs are flux loops enclosing the plasma poloidally. They are sensitive to changes in the toroidal magnetic flux, which is dominated by the main magnetic field generated by the non-planar and planar superconducting field coils of W7-X. If a plasma is generated, the diamagnetic current balancing the pressure gradient of the plasma will reduce the magnetic field in the plasma region, thus changing the magnetic flux through the DL.

This flux change $\Delta \Phi$ can be expressed by a term

$$
\Delta \Phi_{\text {dia }}=\frac{-\mu_{0} W}{3 \pi R B_{0}}
$$

which is linear in the plasma energy $W$, and where $R$ is the major radius of the torus, and $B_{0}$ is the main magnetic field. There are additional terms depending on the plasma current and on the vacuum rotational transform: In an approximation for large aspect ratio, low beta and low shear,

$$
\Delta \Phi=\Delta \Phi_{\text {dia }}+\frac{\pi \mu_{0} t}{R} \int_{0}^{a} j_{\phi}(r) r^{3} \mathrm{~d} r+\frac{\mu_{0} I_{\mathrm{p}}{ }^{2}}{8 \pi B_{0}}
$$




\begin{tabular}{|c|c|c|c|c|c|c|}
\hline \multirow[t]{2}{*}{ Type of coil } & \multicolumn{3}{|c|}{ Location } & \multirow{2}{*}{$\begin{array}{l}\text { Overall } \\
\text { number } \\
\text { of units }\end{array}$} & \multirow{2}{*}{$\begin{array}{l}\text { Number of seg- } \\
\text { ments or of single } \\
\text { coils per unit }\end{array}$} & \multirow{2}{*}{$\begin{array}{l}\text { to be installed } \\
\text { later }\end{array}$} \\
\hline & toroidally & IV & $\mathrm{OV}$ & & & \\
\hline Diamagnetic loop & $\mathrm{BP}$ & $x$ & & 2 & - & - \\
\hline $\begin{array}{l}\text { Diam. loop with } \\
\text { comp. coils }\end{array}$ & $\mathrm{TP}$ & $x$ & & 2 & $\begin{array}{l}1 \text { main loop }+ \\
4 \text { comp. coils }\end{array}$ & 1 \\
\hline Rogowski coil & $\mathrm{TP}$ & $x$ & $x$ & 2 & $1 \mathrm{IV}+1 \mathrm{OV}$ & 1 coil IV \\
\hline \multirow[t]{2}{*}{ Segm. Rogowski coil } & $\mathrm{TP}$ & $x$ & $x$ & 2 & $8 \mathrm{IV}+8 \mathrm{OV}$ & 1 set IV of 8 segments \\
\hline & $\mathrm{BP}$ & $x$ & $x$ & 3 & $11 \mathrm{IV}+11 \mathrm{OV}$ & 3 segments of 1 set IV \\
\hline \multirow[t]{4}{*}{ Saddle coil } & TP bottom & & $x$ & 10 & - & - \\
\hline & TP top & & $x$ & 10 & - & - \\
\hline & BP bottom & & $x$ & 10 & - & - \\
\hline & BP top & & $x$ & 10 & - & - \\
\hline \multicolumn{7}{|l|}{ Mirnov coil } \\
\hline full pol. array & $\mathrm{TP}$ & $x$ & & 4 & $41 / 33 / 26(2 \times)$ & $\begin{array}{l}17 \text { out of } 33 \text { coils } \\
\text { of } 1 \text { unit }\end{array}$ \\
\hline \multirow[t]{2}{*}{ poloidal array } & BP outboard & $x$ & & 2 & $7 / 8$ & all \\
\hline & BP inboard & $x$ & & 2 & $4 / 5$ & - \\
\hline \multirow[t]{2}{*}{ toroidal array } & outboard & $x$ & & 1 & 8 & all \\
\hline & inboard & $x$ & & 1 & 9 & 2 coils \\
\hline
\end{tabular}

Table 1: Magnetic diagnostics in W7-X, as shown in fig. 1. The location of each unit of coils is characterised toroidally by "TP" for triangular plane or "BP" for bean-shaped plane (referring to the plasma cross section in these planes). For the saddle coils, "TP" is a small sector of the half module at the $T P$, whereas " $B P$ " is the rest of the half module up to the BP. "IV" / "OV" designate inside and outside the $P V$, respectively. With "unit" we refer to a set of coils the signals of which will often be used together. For segmented Rogowski coils, these are all segments in one half module located either in the TP or close to the BP. For the Mirnov coils, these are coils forming either a poloidal or a toroidal array for wavenumber resolution of MHD modes. Each diamagnetic loop, compensation coil, continuous Rogowski coil and individual segment of a segmented Rogowski coil contains two electrically independent windings for redundancy.

[11], where $t$ is the rotational transform of the magnetic configuration (assumed to be constant), $a$ is the minor radius of the torus, $j_{\phi}$ is the toroidal component of the net plasma current density at minor radius $r$, and $I_{\mathrm{p}}$ is the total toroidal plasma current. The two additional terms are small compared with $\Delta \Phi_{\text {dia }}$, if the toroidal plasma current is small. In a real stellarator, additional terms exist due to the three-dimensional geometry of the magnetic field contributions generated by plasma currents. Part of these additional terms related to the toroidal plasma current can be minimised by arranging the DL perpendicular to the plasma axis and thus in leading order perpendicular to a possible toroidal plasma current. A detailed derivation of all these terms can be found in [12]. In a full analysis of the signals from the MEDs, the plasma energy will be calculated in the framework of an equilibrium reconstruction, where all corrections will be taken into account numerically.

If the plasma pressure is anisotropic, it is the pressure component perpendicular to the magnetic field which determines the signal measured by a DL [13] (see also section 2.1.3). 
Since the magnetic flux through a DL is dominated by the main field, even small variations in the field coil currents during a discharge can easily spoil the plasma signal. For the same reason, the design of a DL must offer sufficient stability against shifts, vibrations or deformation, which could change the area perpendicular to the main magnetic field spanned by the loop. This is of special concern if a certain accuracy is to be attained in long-pulse experiments, because the measurement signals are integrated together with such unwanted "error" signals.

Part of such error signals can be avoided by arranging a compensation coil (CC) together with a DL, or by a double loop approach [14]. The CC (the approach chosen for W7-X) measures the toroidal magnetic flux in the same plane as the DL, but sparing the plasma cross section. This is typically realised by a flux loop spanning the area between plasma and PV. Usually, the winding areas of DL and CC are chosen equal, such that their signals can be subtracted from each other in an analog serial circuit in order to eliminate changes of the main magnetic field. In W7-X, the signals of both the CCs and the DL are digitised separately, and the compensation will be performed numerically.

A second reason for CCs or an equivalent approach is that a real DL does not only enclose the plasma volume, as an ideal DL would do. Its time resolution can therefore be limited by the poloidal eddy currents in the PV. These decay with the $L / R$ time of the PV, which is $\sim 10-20 \mathrm{~ms}$, if the DL is arranged outside the PV or inside but close to the PV wall. Thus, fast changes of the plasma energy would not be observable. E. g., edge localised modes (ELMs) [15] in tokamaks can reduce the plasma energy by up to $10 \%$ within $\sim 100 \mu \mathrm{s}[16,17]$. Likewise, confinement time investigations in heating power switch-off experiments would become difficult if the confinement time is smaller or in the order of the $L / R$ time of the PV. CCs allow to reconstruct a faster time scale in the change of the plasma energy (see section 4.2). For W7-X, a time resolution of $1 \mathrm{~ms}$ was specified for the CCs.

All 4 DLs of W7-X are located inside the PV, 2 in TPs and 2 close to BPs (see fig. 1). CCs could only be realised with the TP DLs. For the BP DLs insufficient space between PV and WPEs prohibited such a solution. The windings of the TP DLs are arranged in a plane perpendicular to the magnetic axis in order to minimise the impact of net toroidal plasma currents on the signal of the DL (see above).

\subsubsection{Saddle coils}

The SCs are flux loops on the PV with sections in toroidal direction close to the torus inboard and outboard midplane and connecting sections in poloidal direction, resembling the outline of a saddle. Due to their geometry, they are therefore collecting the magnetic flux radially crossing the PV. In contrast to the DLs, which collect magnetic flux due to poloidal currents, the currents at the origin of the flux collected by the SCs are toroidal ones generating poloidal and radial magnetic field components. Two contributions to the toroidal component of the current density are distinguished.

The first contribution is due to the condition to keep the total current density sourcefree: According to the ideal MHD force balance, the pressure gradient of the plasma is 
balanced by the diamagnetic current $\boldsymbol{j}_{\text {dia }}$,

$$
\boldsymbol{j}_{\mathrm{dia}}=\boldsymbol{j}_{\perp}=\frac{1}{B^{2}} \boldsymbol{B} \times \nabla p .
$$

In toroidal geometry, $\boldsymbol{j}_{\text {dia }}$ in general varies on a magnetic flux surface, e.g., due to the variation of the magnetic field strength $|B|$ at different poloidal positions. In strongly shaped stellarators, the variation of $\nabla p$ may lead to an even stronger variation of $\boldsymbol{j}_{\text {dia }}$ than the variation of $|B|$. The diamagnetic current in itself is therefore not source-free. Only an additional (force-free) current component parallel to the magnetic field will ensure

$$
\nabla \cdot \boldsymbol{j}=\nabla \cdot\left(\boldsymbol{j}_{\mathrm{dia}}+\boldsymbol{j}_{\|}\right)=0
$$

For a torus with circular plasma cross section, the resulting toroidal current density will exhibit a dipolar structure with its maximum values in the torus mid-plane with opposite directions at the torus inboard and outboard sides. This contribution to the toroidal current density is called PS currents and will generate a vertical magnetic field, which modifies the plasma equilibrium such that the magnetic flux surfaces are shifted along the major radius to the torus outboard side (Shafranov shift [18]). The PS currents do not contribute to the net toroidal current.

The diamagnetic current increases with plasma pressure. That is why the integrated signal of a DL can be used as a measure of the plasma energy (see section 2.1.2). For a fixed magnetic configuration, the PS currents are also proportional to the plasma energy. The integrated signals of SCs can therefore be used to determine the plasma energy as well.

One of the major goals in the optimisation of the Wendelstein line of stellarators [9] is the reduction of the PS currents relative to the diamagnetic currents in order to achieve stiff finite- $\beta$ configurations, i. e., plasma shape and position should be only weakly dependent on the plasma pressure (low Shafranov shift). The SCs can be used to verify the achievement of this goal.

The analysis of plasma equilibria with non-isotropic pressure shows that the diamagnetic currents depend mainly on the pressure component perpendicular to the magnetic field, $p_{\perp}$. Only corrections of higher order also depend on $p_{\|}$. The diamagnetic signal therefore mainly represents $p_{\perp}$. This is no longer true for the divergence of $\boldsymbol{j}_{\text {dia }}$, which is the source of the PS currents. Here, $p_{\perp}$ and $p_{\|}$both contribute in the same order of magnitude [13]. By comparing the measurements from DLs and from SCs, pressure anisotropy can therefore be detected, if the measurement accuracies are sufficiently high, as was demonstrated in LHD [19, 20].

The second contribution to the toroidal current component is due to source-free current densities parallel to the magnetic field, which add up to a net toroidal current, e.g., the bootstrap current or externally driven currents. In a simplified picture with the net toroidal current centred relative to the SC contours, the magnetic field generated would not contribute to a net flux through an SC. Deviations from this simple picture are due to the 3D magnetic configuration and PV shape, or due to a radial shift of the net toroidal current with respect to the SC.

In addition, the amplitude of the PS currents is inversely proportional to the rotational transform, which will, in turn, be modified by a net toroidal current and its radial 
distribution. This is a further mechanism how the SC signals are influenced by a net toroidal current and another reason for a deviation from the DL signals, which depend less on the internal current density distribution. Hence, in reality, the SC signals will depend on the net toroidal current.

To summarise, the SC signals contain more information on the plasma profiles than the DL signals, which makes their interpretation more difficult. Full advantage of the complete information contained in the SC signals can only be taken in the framework of an equilibrium reconstruction, as mentioned in section 2.1.2.

In each HM of W7-X, four SCs are installed, two on the upper part of the PV and two on the lower part (black in fig. 1). One upper and one lower SC is located adjacent to the TP, spanning $\sim 0.7 \mathrm{~m}$ in toroidal direction. The other two SCs cover the remaining $\sim 2.4 \mathrm{~m}$ sector of the HM up to the BP. This pattern covers all ten HMs of W7-X, with minor differences between the HMs due to different port versions. The SCs are thus the only magnetic diagnostic of $\mathrm{W} 7-\mathrm{X}$ which reflects the full tenfold symmetry of the magnetic configuration.

\subsubsection{Rogowski coils (including segmented Rogowski coils)}

According to Ampère's law,

$$
\mu_{0} \int_{A} \boldsymbol{j} \cdot \mathrm{d} \boldsymbol{A}=\oint_{\partial A} \boldsymbol{B} \cdot \mathrm{d} \boldsymbol{s},
$$

where $A$ is some surface bounded by the curve $\partial A$. By integrating the magnetic field component along a closed loop $\partial A$, we therefore obtain the total current passing through the loop. A change in $\oint_{\partial A} \boldsymbol{B} \cdot \mathrm{d} \boldsymbol{s}$ is measured by a closed solenoid along $\partial A$ with uniform winding area density, such that $\mathrm{d} / \mathrm{d} t \boldsymbol{B} \cdot \mathrm{d} \boldsymbol{s}$ is equally weighted along the entire loop $\partial A$. Such a solenoid is called Rogowski coil (RC). An RC encircling the plasma in poloidal direction will therefore measure changes in the total toroidal plasma current.

The minimisation of the net toroidal plasma current has been one of the optimisation criteria of W7-X [9]. Because W7-X is a low shear device, already small net toroidal currents will change the rotational transform $t$ at the plasma boundary, shifting the location of the naturally occuring boundary islands, which are crucial for proper divertor operation (at $B=2.5 \mathrm{~T}$, the cylindrical approximation for a major radius of $5.5 \mathrm{~m}$ and a minor radius of $0.5 \mathrm{~m}$ predicts a change of $t$ by 0.0176 for a net toroidal plasma current of $10 \mathrm{kA}$ ). The RCs should therefore be sufficiently sensitive to measure plasma currents of below $1 \mathrm{kA}$, while still providing the necessary measurement range, for which $200 \mathrm{kA}$ was specified (although the operational scenarios of W7-X aim at small toroidal currents, depending on the assumptions for transport simulations, net toroidal currents of up to $\sim 150 \mathrm{kA}$ are estimated, e.g. see [21]).

The limited space did not allow to install pick-up coils measuring the poloidal magnetic field locally with a sufficiently large effective winding area and, at the same time, sufficiently low self-inductance. We therefore use the concept of segmented Rogowski coils (SRCs), which integrate the poloidal magnetic flux over some poloidal length, thus providing sufficient sensitivity.

This is why several of the RCs are split poloidally into different sections, forming sets 
of SRCs which provide information on the poloidal distribution of the toroidal component of the current density. The PS currents can therefore also be measured by this diagnostic.

Electric currents might also flow in the PV wall, either induced by changing plasma currents, or even continuous as thermal currents between different divertor modules. From the signal of a single SRC segment, either at the outside or at the inside of the PV, a distinction between toroidal currents in the plasma and in the PV wall is not possible. The SRC segments are therefore arranged in pairs, located in the same poloidal and toroidal location, one at the outside of the PV and one at its inside. The comparison of the signals from the members of these pairs allows to distinguish between currents in the plasma and in the PV wall.

In addition, the strong variation of the plasma cross section between $\mathrm{BP}$ and $\mathrm{TP}$ offers the chance to obtain some information on the radial distribution of the toroidal component of the current density: Qualitatively, close to the BP, due to the high vertical elongation of the plasma cross section, a current on flux surfaces close to the magnetic axis will generate a lower flux in the RC segments at the top and at the bottom than in the RC segments located close to the midplane. The same poloidal current distribution on flux surfaces close to the plasma edge will generate a different distribution of magnetic flux in the individual $\mathrm{RC}$ segments. In the $\mathrm{TP}$, in contrast, the distribution of the magnetic flux in the individual RC segments does not depend as much on the radial location of the toroidal current density component generating this flux. From the analysis of SRC signals in $\mathrm{BP}$ and $\mathrm{TP}$, qualitative information on the peakedness of the radial distribution of the toroidal component of the plasma current can therefore be inferred. Similarly, the pressure profile can be qualitatively assessed. The accuracy and limitations of an equilibrium reconstruction based on the present set of W7-X MEDs is investigated in [7].

Finally, the sum of the signals from a (poloidally) complete set of SRC segments will contain the same information as the signal from a CRC, although, in the design realised in W7-X, the gaps between adjacent segments of the SRCs had to be chosen larger than in the CRCs.

In contrast to the SCs, a fully symmetric distribution of RCs in all modules could not be realised. There exist two CRCs in TPs, two SRCs in TPs and three SRCs close to BPs (see fig. 1). In the TPs, each SRC consists of 8 segments, which are located almost in the same poloidal plane. Close to the BPs, each SRC consists of 11 segments, of which 7 are located almost in the same poloidal plane. These leave a poloidal gap at the torus inboard side, where the space between PV and WPEs is insufficient. The remaining 4 segments are shifted toroidally along the magnetic field lines (for W7-X standard configuration), such that a complete poloidal coverage in magnetic flux coordinates is granted.

As discussed above, there are pairs of corresponding segments on the outside and on the inside of the PV, such that electric currents in the PV can be distinguished from plasma currents. Likewise, the CRCs consist of corresponding pairs inside and outside the PV. In addition to avoiding a misinterpretation of measurement signals due to toroidal currents in the PV wall, this arrangement will make the RCs the only source of information about such currents in the PV. 


\subsubsection{Mirnov coils}

In contrast to the quantities measured by the MEDs, no particular symmetry can be assumed for the MHD modes to be investigated by the Mirnov coils. Rather, it is the task of this diagnostic to identify the spatial structure of different modes. The highest frequencies of the order of $400 \mathrm{kHz}$ are expected for different Alfvén eigenmodes, in particular mirror-induced Alfvén eigenmodes and helicity-induced Alfvén eigenmodes [22]. The target for the Mirnov coils therefore is to measure magnetic fluctuations in the frequency range from few $\mathrm{kHz}$ up to $\sim 1 \mathrm{MHz}$ with good poloidal and toroidal resolution.

MHD modes are spatially non-uniform and are in toroidal geometry usually characterised, apart from their frequency, by their poloidal and toroidal mode numbers, $m$ and $n$. To determine these mode numbers, full poloidal arrays of up to 41 coils are provided in four TPs. Close to the BPs, only partial arrays are provided with sets of 4-8 coils on the torus outboard and inboard sides in two modules. For full toroidal arrays of densely spaced Mirnov coils, an excessive number of coils would be required. We therefore rely on the observation that MHD modes occur coherently in the entire device. Since our poloidal arrays are well distributed around the whole torus, it is sufficient to determine the helicity of the modes in a limited toroidal region. In this region, Mirnov coils are distributed toroidally with increasing separation, such that many different multiples of the smallest separation occur with a minimum number of coils. One such toroidal array is located on the torus outboard side, a second one on the torus inboard side. The entire distribution of Mirnov coils is suitable for a simultaneous analysis of their signals, which will reveal the spatial mode structure (see, e. g., [23]).

Since the amplitude of a magnetic perturbation with poloidal mode number $m$ decays $\propto r^{-(m+1)}$ with the distance $r$ from the plasma axis, Mirnov coils must be located close to the plasma boundary. Furthermore, to minimise the damping of the magnetic perturbations by eddy currents, no electrically well conducting structure should be located between the plasma and the Mirnov coil. At least, such a conducting structure should have a gap with appropriate orientation.

The fluctuating components of the magnetic field due to MHD modes and turbulence are caused by plasma currents, which flow mainly along the magnetic field. The resulting magnetic field variations have therefore components mainly perpendicular to the unperturbed field direction, i.e., approximately poloidal and radial components, whereas the variation of the field component parallel to the unperturbed magnetic field is expected to be much smaller. All W7-X Mirnov coils are therefore arranged to measure changes in the poloidal magnetic field component.

With this set of Mirnov coils, we should be able to determine the spatial structure of MHD modes and to investigate for each mode whether its amplitude varies between BP and TP (local ballooning or anti-ballooning due to the variation of the magnetic curvature within one module, or depending on the location in a magnetic mirror field), or between torus outboard and inboard sides (ballooning or anti-ballooning on a scale of $\pi R \sim 15 \mathrm{~m}$ ).

In order to avoid resonances in the Mirnov coil circuits in the frequency range of interest, the inductance of the Mirnov coils and the capacitance of their signal cables should be low, and the signal cables should be short or terminated with a matching impedance. 


\subsection{Location inside or outside the plasma vessel}

For the placement of the different pick-up coils, positions inside or outside the PV were considered. Both options have advantages and disadvantages. Since the magnetic fields to be measured are generated by currents within the plasma, placing the pick-up coils close to the plasma without many structures between plasma and pick-up coil is favourable.

Outside the PV, the SCs and part of the CRCs and SRCs are installed. They are located directly on the surface of the PV, i. e., in the cryovacuum of the superconducting coils, but on the "warm side" of the thermal insulation (for a description of the W7-X cryospace see [24, 25]). Inside the PV, most of the sensors are placed between PV and WPEs or between PV and divertor or baffle. The WPEs are, depending on the thermal load to be expected locally, either groups of graphite tiles or steel panels. Both types of WPE will be water-cooled during long-pulse operation. A more detailed description of these elements can be found in $[26,27,28]$. The Mirnov coils are installed directly behind the gaps between WPE graphite tiles and on the plasma side of steel panels.

The advantages and disadvantages of locations inside and outside the PV are:

- Outside the PV, the sensors are protected from the potentially harsh conditions inside the PV, where high thermal load and high microwave radiation level require appropriate protection and either active cooling (not realised here) or good thermal contact to actively cooled components (see sections 3.2 and 3.3).

- In spite of these measures, still considerable temperature variation of the in-vessel sensors during long-pulse discharges is expected (up to $\sim 200 \mathrm{~K}$ ), whereas outside the PV, the temperature variation during long-pulse discharges is expected to stay below $\sim 50 \mathrm{~K}$.

- The space outside the PV between PV and thermal insulation is very limited. Basically, it is the space left between the cooling pipes of the PV (see fig. 2). More space (at least in some locations) is available inside the PV.

- The time resolution of magnetic sensors outside the PV is limited by the time scales of the PV for the decay of induced currents, which are $\sim 17 \mathrm{~ms}$ for poloidal and $\sim 47 \mathrm{~ms}$ for toroidal currents [29]. Inside the PV the time resolution can be better (depending on the type of sensor and its precise location - see the specific discussions in sections 4,6 and 7 ).

- The pick-up coils cannot distinguish between electric currents in the PV wall and inside the PV. In particular, an ideal RC inside the PV measures only the toroidal plasma current, whereas an RC outside the PV is also sensitive to toroidal currents in the PV. Therefore, complementary sets of CRCs and SRCs are installed inside and outside the PV, such that measurements of complementary coils will provide information on electric currents in the PV. Moreover, for the SRCs such an approach is necessary anyway, because also the signals of the in-vessel segments may be severely influenced by toroidal currents in the plasma vessel. 


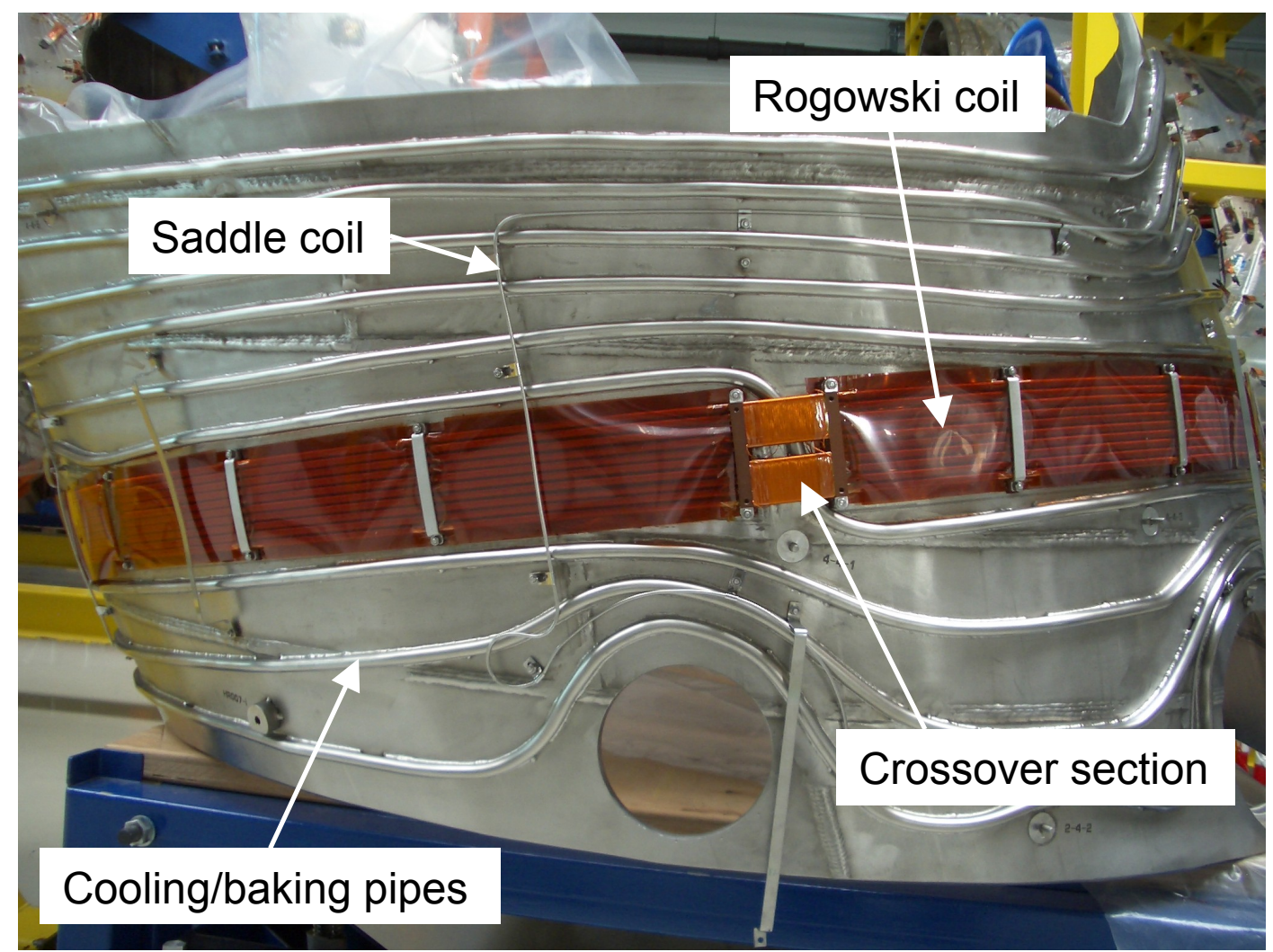

Figure 2: Rogowski coil and saddle coil mounted on one of the small sectors of the plasma vessel. The thermal insulation is installed on top of this arrangement. The space between plasma vessel and thermal insulation is very limited. Therefore, the Rogowski coil segments are installed mostly between the pipes. At pipe crossings, special crossover segments with reduced height are used, which however have the same winding area density as the rest of the Rogowski coil. The pipes are welded to the plasma vessel and are used for baking the plasma vessel and for cooling it during plasma operation.

- The sensors and signal cables located between thermal insulation and PV are, in practice, no more accessible after the assembly of W7-X. Sensors and signal cables inside the PV will be accessible, if a limited number of WPEs is removed.

Based on these considerations, we decided to install the DLs together with the CCs inside the PV, which will provide the required time resolution (in the TPs, the DLs are located on the plasma side of the CCs, some 80-90 mm inside the PV, which reduces the impact of the eddy currents in the PV on the time resolution of the DL). The SCs are only installed outside the PV, since high time resolution for the diamagnetic energy is provided by the DLs together with their CCs, and some information on PS currents is also provided by the SRCs. The SCs with their fully symmetric arrangement (see section 2.4) will then provide information on asymmetries with a time resolution sufficient for most changes of the plasma configuration.

The Mirnov coils must be installed inside the PV because of the fast time scale of MHD modes, as compared with the time constants of the PV. 


\subsection{Redundancy}

Since the measurements of the MEDs are essential for analysing the experiments on W7-X, the failure of one or several pick-up coils must not result in a significant loss of information. To achieve redundancy,

- each "coil" contains two electrically independent windings with a separate pair of signal wires and separate electronic and data acquisition (ADC) channels (this is the case for the DLs, CRCs and SRCs)

- each type of coil exists in more than one half module (this is the case for both BP and TP types of all MEDs)

- the upper and the lower coils of a pair of SCs encircle a rather similar magnetic flux since their toroidal sections run close to each other, close to the midplane; this provides an additional level of redundancy.

\subsection{Asymmetries}

Although, in theory, the measurement results of the MEDs from equivalent positions in different HMs should only differ due to the measurement uncertainty, the ten-fold symmetry may be broken in the real device. Reasons for asymmetries of the magnetic field could be, e.g., shape and position errors of the field coils, or ferromagnetic components close to the torus, although the use of ferromagnetic material is strictly controlled and assessed. Moreover, position errors of the target elements could result in differences in target loads (as observed in the divertor phase of W7-AS [30]), which may also result in symmetry-breaking electric currents in the plasma edge and scrape-off layer. Although the measurement of such asymmetries will be the task of dedicated other diagnostics [31], the MEDs may contribute information to these measurements. Lastly, different plasma heating power or methods in different modules of W7-X could result in asymmetries in the pressure anisotropy in high-mirror magnetic configurations (see the discussion in section 2.1.3).

In addition, the measurement with equivalent magnetic sensors in equivalent positions will provide a valuable indication of the measurement uncertainty, independent of theoretical considerations.

Therefore, the original design comprised two DLs in BPs, two DLs in TPs, two CRCs, three SRCs in BPs, two SRCs in TPs, and a fully symmetric set of 40 SCs, four per half module

Due to limited resources, one TP DL, one in-vessel CRC, one in-vessel TP SRC and several segments of one in-vessel BP SRC will not be installed before the start of W7-X operation. 


\section{$3 \quad$ Major challenges}

\subsection{Long-pulse integration}

Since only changes of the magnetic flux through the winding area of a pick-up coil will induce a voltage in the coil, magnetic equilibrium diagnostics based on pick-up coils require the integration of the coil signals from the start of a plasma discharge, either electronically or numerically, in order to obtain the desired quantities at every moment of the discharge. In consequence, every offset on the voltage to be integrated will result in an error of the integrated signal, increasing linearly with the time from the start of integration. Such an offset can be determined in a time interval before the start of integration and can then be corrected for. However, if the offset in itself is subject to a drift, the compensation will no longer be correct.

A second source of error are changes of the magnetic flux through the winding area of a pick-up coil due to shifts or deformation of the coil. In this case, a signal is induced even if the quantity of interest does not change. This problem is aggravated if the major fraction of the magnetic flux penetrating the winding area of the pick-up coil is not generated by currents in the plasma but rather by the field coils of the device. This is most pronounced for the DLs.

\subsubsection{Offset voltages generated within the electronics}

The characteristics of many electronics components depend on their temperature, which can give rise to drifts in the offset of an amplifier or ADC. The temperature dependence is comparatively low if an element like a transistor is used as a switch rather than an amplifier. Therefore, one of the first stages of the electronics for the magnetic equilibrium diagnostics is a chopper which periodically reverses the polarity of the input signal. The influence of all offset drifts in the electronics after the chopper can thus be eliminated. A constant offset voltage external to the electronics can be determined before the start of the integration and then be compensated. Drifts as low as $30 \mu \mathrm{Vs}$ over 10 min were demonstrated with this principle [4]. The prototype developed for $\mathrm{W} 7-\mathrm{X}$ reaches $\sim 100 \mu \mathrm{Vs}$ over $1000 \mathrm{~s}$, and $\sim 50 \mu \mathrm{Vs}$ if the compensation of the external drift can be done using the offset signals both before the start and after the end of the integration interval.

\subsubsection{Thermovoltages}

Contact voltages arise if two different conductors are in contact. These contact voltages are specific for the pair of conductor materials and depend on temperature. In a typical wire connector, there will be a pin and a jack crimped to two sections of wire. Even if the wire sections are identical, the wires may be plated (e.g., $\mathrm{Cu}$ wires plated by $\mathrm{Ag}$ or Ni), pin and jack may be of different material and plated in itself, such that easily 5 different interfaces may occur in such a connection. If temperature gradients along the connection and a temperature difference between neighbouring connectors exist, a constant voltage will be generated within the circuit affected. If the temperature differences change during long-pulse integration, even an offset compensation as described in section 3.1.1 will fail. 
3.1.2.1 Magnitude Thermovoltages at $0^{\circ} \mathrm{C}$ are, e.g., $\sim 20 \mu \mathrm{V} / \mathrm{K}$ between $\mathrm{Fe}$ and $\mathrm{Cu}$ and $\sim 100 \mathrm{nV} / \mathrm{K}$ between $\mathrm{Cu}$ and $\mathrm{Ag}$. Hence, even the thermovoltage between a single pair of $\mathrm{Cu}-\mathrm{Ag}$ interfaces at only $1 \mathrm{~K}$ temperature difference, integrated over $1000 \mathrm{~s}$, is as large as the drift of the W7-X prototype integrator for such a time interval. This is also the same order of magnitude as the sensitivity required for several of the pick-up coil circuits (see sections 4-6). It is therefore essential to minimise the thermovoltages in the circuits of the MEDs.

It is difficult to quantify in advance the temperature differences, e.g., within a multipin connector in a vacuum feedthrough with cooling pipes running by closely, or at the connection between a pick-up coil and its signal cable within the PV. We therefore set up an experiment aiming to compare the thermovoltages between (1) pin-and-jack connections, (2) crimped connections, (3) welded and cold welded connections. To this end, we produced a number of specimens, each with two sections of winding wire or signal cable (silver-plated $\mathrm{Cu}$ wire), which were connected in different ways. Close to the connection under test, the wire sections were routed through heating baths, keeping one section at $\sim 20^{\circ} \mathrm{C}$, while the other section was heated to temperatures of $\sim 120^{\circ} \mathrm{C}$. The temperatures of the heating baths were measured by thermocouples, the voltage between the far ends of the wire sections (which were at equal temperatures) was measured by a nanovoltmeter.

To summarise the results, three pin-and-jack connections (differing in the material and plating of the pins and jacks) yielded voltages of $200-360 \mathrm{nV} / \mathrm{K}$. Three crimp connections (all $\mathrm{Cu}$, but with or without silver plating on the crimp sleeves and on the wire) yielded voltages of $1.1-3.3 \mathrm{nV} / \mathrm{K}$. In the crimp connections, there was always direct contact between the two wire sections, the crimp sleeves only served to press the wires together. Finally, the different welded and cold welded connections yielded voltages of $0.5-1.6 \mathrm{nV} / \mathrm{K}$. For comparison, the measurement was also performed with one continuous wire, yielding $0.25 \mathrm{nV} / \mathrm{K}$.

We emphasise that these numerical values are specific for the test arrangment: The temperature was not measured directly at the interfaces, where it depends on the thermal conductance across the connection, which may be one reason for the poor performance of the pin-and-jack connections. Nevertheless, even in this aspect, the experiment is representative for the situation in $\mathrm{W} 7-\mathrm{X}$.

In consequence, we chose crimped connections between the pick-up coils and the signal cables. We chose welding, where 11-24 connections in series had to be established in the DLs (see section 4).

3.1.2.2 Reducing thermovoltages in plug connectors In some locations, plug connections could have only been avoided by excessive efforts. One such example are the vacuum feedthroughs. As discussed before, an offset due to thermovoltages in a circuit with a pair of connectors will only occur, if the temperature differs between the interfaces in the two connectors (with equal materials). The reason for this could be a temperature gradient across a multi-pin connector. If each of the wires is split and routed through two connections and the resulting four connections are interlaced, as shwon in fig. 3, a mere linear temperature gradient will no longer cause a thermovoltage offset in the circuit. This scheme is realised at the vacuum feedthroughs of the DLs, CRCs and SRCs. 


\section{multi-pin connector}
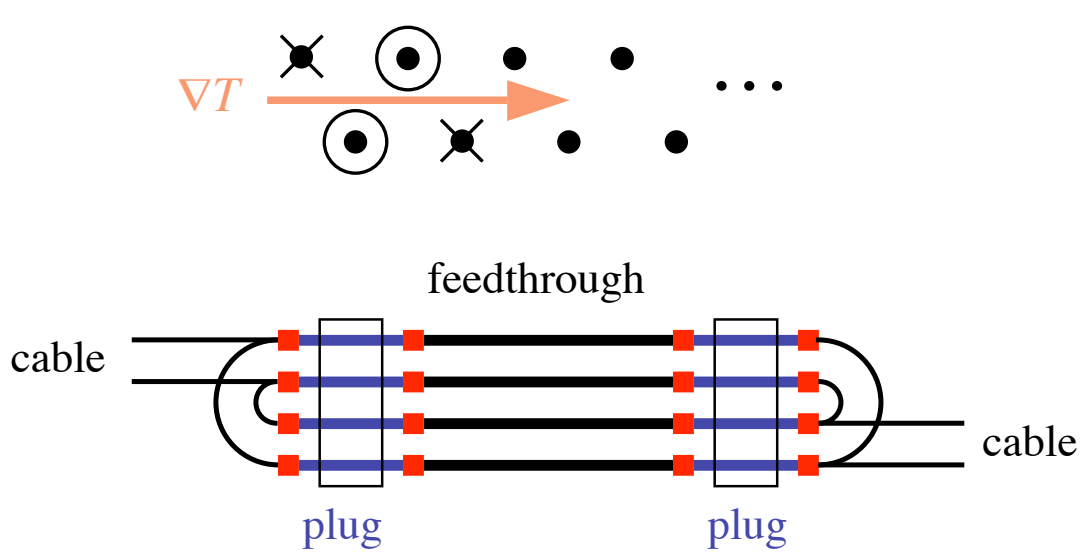

Figure 3: In order to reduce the effect of temperature gradients in a bipolar connection, four connectors instead of two are used (top, here the arrangement for a multi-pin connector is shown, and an arbitrary temperature gradient is indicated). At bottom, a scheme is sketched how to connect a bipolar cable to the four pins in the example of a feedthrough. The interfaces between the conductors, which may give rise to thermovoltages, are marked in red.

As a further measure to reduce thermovoltage differences between two connectors, they can be surrounded by a block of good thermal conductivity (e.g., from $\mathrm{Cu}$ or $\mathrm{Al}$ ), such that temperatures between the two connectors are equalised. This scheme is realised at the vacuum feedthroughs of the SCs.

\subsubsection{Dynamic range}

The measurement accuracy of the MEDs is specified in terms of diamagnetic energy, or plasma current, or a similar quantity, i. e., essentially, in terms of a certain magnetic flux change $\Delta \Phi_{\min }$ through a pick-up coil. There is also some maximum magnetic flux $\Delta \Phi_{\max }$ due to plasma currents, which can be estimated for each pick-up coil. On the one hand side, we want to record a flux change by a large fraction of $\Delta \Phi_{\max }$ within a brief time $\delta t$, if, e.g., the plasma confinement deteriorates suddenly. This defines the maximum voltage induced in the pick-up coil, $U_{\max }=N \cdot \Delta \Phi_{\max } / \delta t$, where $N$ is the coil's winding number. On the other hand side, if $\Delta \Phi_{\min }$ occurs in a long-pulse discharge within an interval $T$, it should still be detectable, even if the discharge is otherwise stationary. This defines the minimum voltage $U_{\min }=N \cdot \Delta \Phi_{\min } / T$, the time integral of which should still be correctly measured. The dynamic range of signals to be detected is therefore

$$
\frac{U_{\min }}{U_{\max }}=\frac{\Delta \Phi_{\min }}{\Delta \Phi_{\max }} \cdot \frac{\delta t}{T}
$$

In W7-X, the measurement accuracy is typically specified such that $\Delta \Phi_{\min } / \Delta \Phi_{\max } \sim$ $10^{-3}$, e. g. $2 \cdot 10^{-3}$ for the diamagnetic energy (see section 4.2 ) or $0.5 \cdot 10^{-3}$ for the plasma current (see section 6.2). For $\delta t$, a conservative assumption is $10 \mathrm{~ms}$, although $20-50 \mathrm{~ms}$ are probably more realistic, and $T=1800 \mathrm{~s}$. The resulting dynamic range is $U_{\min } / U_{\max } \sim$ $2 \cdot 10^{-9} \ldots 10^{-8}$, corresponding to $26 \ldots 29$ bit resolution of the ADC. 
A true resolution better than 16 bit is however difficult to achieve in ADCs. It is also difficult to maintain a noise level of the order of $100 \mathrm{nV}$. In this context, however, it is important to understand the nature of the specification: If we split the measured flux change into a "true" flux change $(\Delta \Phi)_{\text {true }}$ and a flux change due to measurement errors of any kind $(\Delta \Phi)_{\text {err }}$, with

$$
\begin{aligned}
\int_{t_{1}}^{t_{2}} U \mathrm{~d} t=\Delta \Phi & =(\Delta \Phi)_{\text {true }}+(\Delta \Phi)_{\text {err }} \\
& =\int_{t_{1}}^{t_{2}} U_{\text {true }} \mathrm{d} t+\int_{t_{1}}^{t_{2}} U_{\text {err }} \mathrm{d} t
\end{aligned}
$$

the specification is

$$
\left|\int_{t_{1}}^{t_{2}} U_{\text {err }} \mathrm{d} t\right| \leq \Delta \Phi_{\text {min }}
$$

for any time interval $\left[t_{1}, t_{2}\right]$. $U_{\min }$ as calculated above results for the special case $U_{\text {err }}=$ const and $t_{2}-t_{1}=T$.

Therefore, a sufficiently large noise level on the input signal could even solve the problem to measure $U_{\min }$ during a long time interval with insufficient ADC resolution: If random noise with Gaussian distribution and a standard deviation of $\sim 0.5$ bit is added to a constant signal of $10^{-4}$ bit, if the result is then digitised with a rate of $2 \mathrm{MHz}$ and integrated for $1800 \mathrm{~s}$, the correct result is obtained with high accuracy. It remains to be tested whether this concept is already realised due to the "natural" noise level on the signals of the MEDs or can at least be implemented by additional measures.

\subsubsection{Further sources of error in long-pulse integration}

During a long-pulse discharge, the heating of the pick-up coil itself or of the PV may result in a shift or deformation of the pick-up coil. In the presence of the background field of the W7-X field coils, this may lead to a change of the magnetic flux through the winding area of the pick-up coil unrelated to any currents in the plasma. This is a particularly severe perturbation if the flux due to the background magnetic field is significantly larger than the flux due to the plasma currents to be measured, as it is the case for the DLs and their CCs.

It will probably be possible to distinguish such effects from signals due to changes of the plasma currents by a comparison between the signals from the different pick-up coils, taking into account also the readings of the Pt1000 temperature sensors which are integrated into the in-vessel pick-up coils of the MEDs (see sections 4.1.3 and 6.1.3).

\subsection{Microwave stray radiation}

Electron cyclotron resonance heating $(\mathrm{ECRH})$ at $140 \mathrm{GHz}$ is foreseen as the main plasma heating system for W7-X. In certain heating scenarios with low single-pass absorption of the ECR waves, a stray radiation field with considerable power density will be generated in the entire PV, due to the high reflectivity of most wall areas of the vessel. Power 
densities of up to $200 \mathrm{~kW} / \mathrm{m}^{2}$ are expected in those machine modules where the ECR launchers are located [32]. Behind the wall protection elements, the stray radiation power should be lower, and a level of $50 \mathrm{~kW} / \mathrm{m}^{2} \mathrm{cw}$ of microwave power was specified to be tolerated by the components installed there [33].

Most insulators used in conventional cables or wires have a significant absorption for microwaves of $140 \mathrm{GHz}$ and heat up to temperatures above their limit within 10-100 s at such power densities. Even a $\mathrm{Cu}$ braid will heat up to several $100^{\circ} \mathrm{C}$, in spite of the low microwave absorption coefficient of $\mathrm{Cu}$ (see below), because the thin wires of the braid have an insufficient thermal conductance to transmit the heat to a heat sink over more than a few millimetres. One option would be mineral-insulated cables with an outer metal sheath. Used as winding wires, these would, however, severely limit the winding density at least of the Rogowski coils.

The option finally chosen are metal housings of all pick-up coils and their signal cables inside the PV, except for the Mirnov coils. Inside the housings, winding wires and signal cables with polyimide insulation are used. The housings may not have openings larger than half the wavelength of the microwaves. To enable pumping, holes of $0.5-1 \mathrm{~mm}$ size were applied to the housings.

The materials open to microwave irradiation then mainly are $\mathrm{Cu}$, stainless steel (SS), and carbon. The microwave absorption at conducting surfaces depends on the microwave frequency, the conductivity of the material, the angle of incidence, the polarisation relative to the angle of incidence and the surface roughness, if this is comparable with or larger than the skin depth, which is $\sim 0.2 \mu \mathrm{m}$ for $\mathrm{Cu}, \sim 0.5 \mu \mathrm{m}$ for non-magnetic SS, and $\sim 4 \mu \mathrm{m}$ for graphite. In our case, the frequency is fixed and the stray radiation field, generated by many reflections and passing through the plasma, can be assumed to be isotropic and unpolarised. The reported losses of $0.1-0.2 \%$ for $\mathrm{Cu}, 0.6-0.8 \%$ for SS, and $\sim 5 \%$ for graphite $[34,35]$ are valid for perpendicular incidence and rather smooth surfaces. Taking into account that an integration over polarisation and angle of incidence should be performed (for the angular dependence, see [35]), and regarding tests of our components, we used conservative values for the $140 \mathrm{GHz}$ stray radiation absorption of $0.6 \%$ for $\mathrm{Cu}$, $2.6 \%$ for SS, and $10 \%$ for carbon in our design. Deposition layers, as they may occur even behind the plasma facing components due to the erosion of carbon, do not dramatically change the absorption coefficients in the $140 \mathrm{GHz}$ frequency range [36].

For a pick-up coil, the design of a metallic housing must reconcile three contradictory requirements:

1. a low microwave absorption requires a good electric conductivity,

2. a good thermal conductance is necessary to limit the temperature, since the absorbed power is still significant, and

3. the electric conductivity should be low enough to achieve the desired time resolution of the diagnostic, which is limited by eddy currents in the housing when changes in the magnetic flux occur.

For each of the specific shielding designs, as presented in sections 4, 6 and 8, qualification tests in the MIcrowave STray RAdiation Launch facility (MISTRAL) [37, 38] at $140 \mathrm{GHz}$ 
microwave power densities of $\sim 50 \mathrm{~kW} / \mathrm{m}^{2}$ were performed, demonstrating that the winding wires and signal cables inside the shielding remain undamaged in half-hour pulses and are not heated significantly above the temperature of the shielding itself.

In order to verify the designs with respect to point 3 of the above requirements, a magnetic probe test stand (MPTS) was used, consisting of three pairs of Helmholtz coils perpendicular to each other (see fig. 4). Each pair of Helmholtz coils can be operated

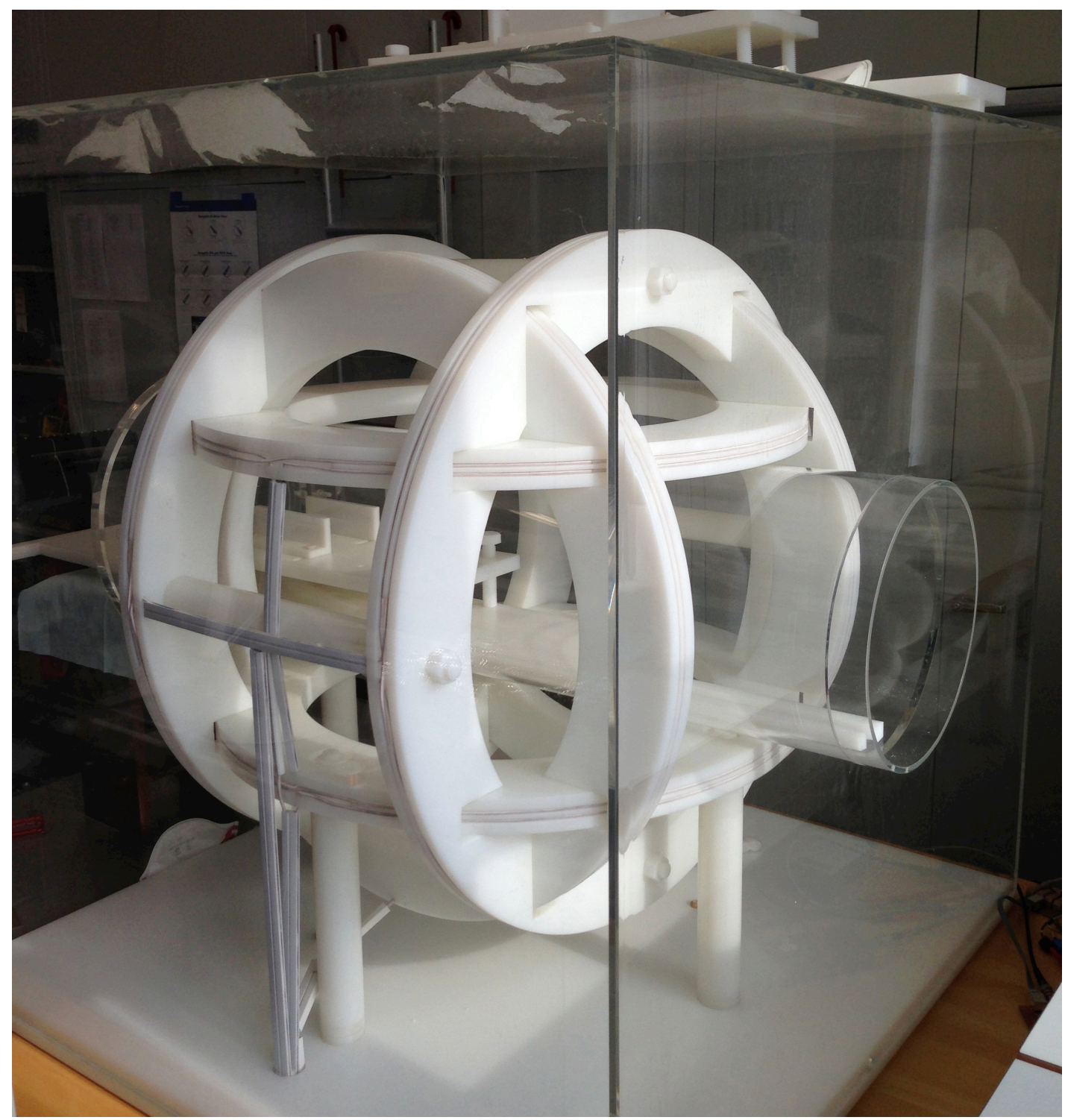

Figure 4: Magnetic probe test stand with three mutually perpendicular pairs of Helmholtz coils for measuring the response of pick-up coils. The coil under test is to be placed in the centre of the test stand, within the acrylic glass tube.

with a harmonic voltage in the frequency range $10 \mathrm{~Hz}-5 \mathrm{MHz}$. The maximum magnetic field in the centre of the MPTS is $100 \mu \mathrm{T}$ up to $10 \mathrm{kHz}$, and $(10 \mathrm{kHz} / f) \cdot 100 \mu \mathrm{T}$ for a frequency $f>10 \mathrm{kHz}$. The Helmholtz coil current is measured using a precision resistor, delivering a reference signal. The signal induced in the coil under test is compared with the reference signal, using a two-phase lock-in technique. As result, amplitude and phase of the transfer function are transmitted to the control computer. In this way, the transfer 
function for a pre-programmed set of frequencies can be measured and compared with the result of a quadratic loop with known winding area, simple geometry and low selfinductance (calibration loop). The entire MPTS was delivered by STL Systemtechnik Ludwig GmbH, Konstanz, Germany [39].

\subsection{Additional thermal load by thermal radiation}

The pick-up coils inside the PV are mostly located behind the WPEs, i. e., panels [28] and graphite tiles with their heat sinks [27]. The graphite tiles are larger than the heat sinks, such that a fraction of their back faces can directly radiate onto components located at their back on the PV wall. Locally, these back faces can reach temperatures of up to $600^{\circ} \mathrm{C}$ under (conservative) assumptions used for the layout of the in-vessel components $[40]$.

Although the emission and absorption coefficients of metallic surfaces for thermal radiation are typically below 0.1 , they may increase to values around $0.3-0.8$ if carbon or hydrocarbon layers are deposited on the surface [40]. The thermal emission coefficient of the graphite tiles is $\sim 0.9$. With these values, local thermal loads of up to $15 \mathrm{~kW} / \mathrm{m}^{2}$ must be tolerated by the in-vessel components of the magnetic diagnostics, in addition to the microwave stray radiation of section 3.2 [40]. "Local" here denotes area sizes of $0.1 \mathrm{~m}$ in poloidal and $1 \mathrm{~m}$ in toroidal direction [41]. Before the start of long-pulse operation, it is planned to install additional thermal shields, which will block the direct lines of sight between the back faces of the graphite tiles and the DLs and in-vessel RCs. E.g., appropriate $\mathrm{Cu}$ shields will reduce the load by thermal radiation by more than a factor of $3[40]$.

The thermal radiation acts only on the side of the components toward the WPEs, whereas the microwave stray radiation impinges on the entire surface, since the spaces between WPEs and PV wall form subcavities with (for the microwaves) highly reflecting walls.

\subsection{Design principles to meet the challenges}

Before describing the detailed design of the different components, we list several design principles which were repeatedly applied. We should note that, for many components, alternative designs were considered, which might also have met the requirements. However, due to limited resources, parallel development and testing of different approaches to a mature state of the design was in most cases not possible. Therefore, the decision for some concept was taken quite early in the design processes.

To screen ECR stray radiation from the winding wires of in-vessel MEDs, metallic shieldings are most efficient, due to their low microwave absorption (see section 3.2). They are also favourable to reflect thermal radiation, as long as no carbon or hydrocarbon layers are deposited on their surfaces (see section 3.3). Still, with the conservative values of section 3.2 , a microwave power load of $\sim 1.5 \mathrm{~kW} / \mathrm{m}^{2}$ on SS surfaces and of $\sim 0.3 \mathrm{~kW} / \mathrm{m}^{2}$ on $\mathrm{Cu}$ surfaces must be tolerated, and the load by thermal radiation on some surfaces may be even higher. This requires a good thermal conductance toward the plasma vessel, which acts as a heat sink. On the other hand side, in metals, electric and thermal conductivity 
are closely related. If large electric currents are induced in the shielding on current paths with a strong inductive coupling to the winding of a pick-up coil, the time resolution of the magnetic sensor will be reduced. In addition, large eddy currents can also result in large electromagnetic forces.

Our first design step is therefore the choice of a shielding material ( $\mathrm{SS}$ or $\mathrm{Cu}$ ), thickness and geometry, such that current paths within the shielding with a strong inductive coupling to the winding of the pick-up coil have a sufficiently high electric resistance, i. e., that the eddy currents induced in the shielding do not limit the time resolution of the pick-up coil below its specifications. The electric conductivity of the shielding is then usually low enough not to exert untolerable electromagnetic forces. At the same time, the thermal conductivity of the shielding is so low that its temperature will exceed the limit of $250^{\circ} \mathrm{C}$ of the winding wire.

The next step is the addition of thermal paths toward the plasma vessel in such an orientation that the resulting current paths do not inductively couple to the coil winding. In addition, currents on these new paths must be limited not to exceed the tolerable electromagnetic forces.

In this task we use the fact that electric currents must flow on closed loops, whereas this is not the case for the thermal flux: A structure which is thermally attached to a heat sink in two positions will have a point of maximum temperature and zero heat flux somewhere between the two attachments. If the thermal (and electric) conductance is small in this area, the heat flux pattern will not be altered much, whereas electric currents can be significantly reduced.

In the design of heat flux paths to the plasma vessel, the heat must be transmitted across interfaces between subcomponents and between our component and the plasma vessel. The heat transmission coefficients at these interfaces strongly depend on the surface structure and on the pressure. In a series of tests, we observed heat transmission coefficients above $8 \mathrm{~kW} /\left(\mathrm{m}^{2} \mathrm{~K}\right)$ for an interface of $200 \mathrm{~mm}^{2}$ between smooth $\mathrm{Cu}$ surfaces at a pressure of the order of $2 \mathrm{MPa}$. However, the PV surface is not as smooth and not even plane. For the in-vessel RCs and the DLs, we therefore use $\mathrm{Cu}$ adapter blocks, with their surfaces toward the PV individually machined after local scans of the PV surface. Graphite layers of $1 \mathrm{~mm}$ thickness are inserted between the adapter blocks and the PV, which, due to their softness, balance the remaining mismatch between PV and adapter block at a significantly lower contact pressure. A heat transmission coefficient of $2 \mathrm{~kW} /\left(\mathrm{m}^{2} \mathrm{~K}\right)$ was reported for such graphite layers at a contact pressure of $0.25 \mathrm{MPa}$ [42]. Tests showed that this pressure is routinely achieved over most of the interface area when the adapter blocks are bolted to the PV surface. By using graphite interlayers, a reduced heat transmission coefficient as compared with an ideal interface between metallic surfaces can hence be reliably achieved at moderate contact pressure and surface quality.

Likewise, the ohmic resistance across the interface may be very low for well matching metallic surfaces, or very high for poorly matching surfaces. With a graphite interlayer of a certain thickness, some minimum ohmic resistance is granted for a certain contact pressure. We therefore use graphite interlayers also in locations where the electric conductance across an interlayer must be limited, but where a minimum heat conductance must be granted and where the use of polyimide or other insulators is impractical because 
they would be exposed to ECR stray radiation.

In sections 4 and 6 , the application of these design principles to the DLs and to the in-vessel RCs is discussed.

\section{Diamagnetic loops}

\subsection{Design and testing}

\subsubsection{Winding and basic design}

The DLs designed for W7-X consist of a rigid support structure, a ribbon cable with polyimide-insulated wires for the winding, an ECR stray radiation protection, and heat conducting elements, which comprise at least the brackets and adapter blocks (see section 3.4) connecting the loop to the plasma vessel.

Depending on the number of turns in the winding, up to four layers of the 25-wire ribbon cable are stacked. The individual wires are micro-welded in series (using a PUK U3 precision welding device of Lampert Werktechnik GmbH, Werneck, Germany, which is based on tungsten inert gas (TIG) welding technology [43]). Since it is impractical to perform the welding once the loop is installed inside the PV, the design must allow to fold the loop for the transport to its final position. A design with two sections connected by a multi-pin plug connector had been considered first, but in the presence of (unavoidable) temperature gradients such a connector would have acted as thermopile, with unacceptable thermovoltages (see section 3.1.2.1).

For redundancy, in each DL and in each CC, the wires of the ribbon cables are connected in such a way that they form two electrically independent windings, each of which is connected to a separate signal cable.

The rigid support structure consists of two side walls, connected by several $3 \mathrm{~mm}$ diametre SS rods. These thin rods also guide the ribbon cable(s) (see figs. 5 and 6), which is laid alternatingly above and under the rods. The distance between the SS walls is kept by bushes or by the (rather stiff) ribbon cable itself.

The very limited space between PV and WPEs did not allow the placement of CCs along all sections of the DL. For those sections, where no reasonable space for a CC exists, due to the narrow space between PV on one side and WPEs or other in-vesel components on the other side, a very flat design was chosen, requiring only $\sim 15 \mathrm{~mm}$ of space (in the following denoted as flat sections). The side walls in these sections consist of $2 \mathrm{~mm}$ SS (see fig. 5). The ECR radiation protection housing consists of $0.2 \mathrm{~mm} \mathrm{Cu}$ sheets, which are individually designed and folded, according to the local shape of the support structure. This "flat" design is employed for the entire length of the DLs in the BPs, where no CCs at all could be accomodated, and for approximately one fifth of the length of each DL in the TPs.

For the time resolution of $1 \mathrm{~ms}$ specified for the CCs (see section 2.1.2), the eddy currents in a $2 \mathrm{~mm}$ SS wall (or, even worse, in a $0.2 \mathrm{~mm} \mathrm{Cu}$ sheet) would be too large (see section 4.1.2). The side walls in these sections were therefore laser cut from a $3 \mathrm{~mm}$ ceramic fibre reinforced ceramic compound (Keramikblech ${ }^{\circledR}$ FW30 of Walter E. C. Pritzkow Spezialkeramik, Filderstadt, Germany [44], see fig. 6). In order to obtain a reasonable 


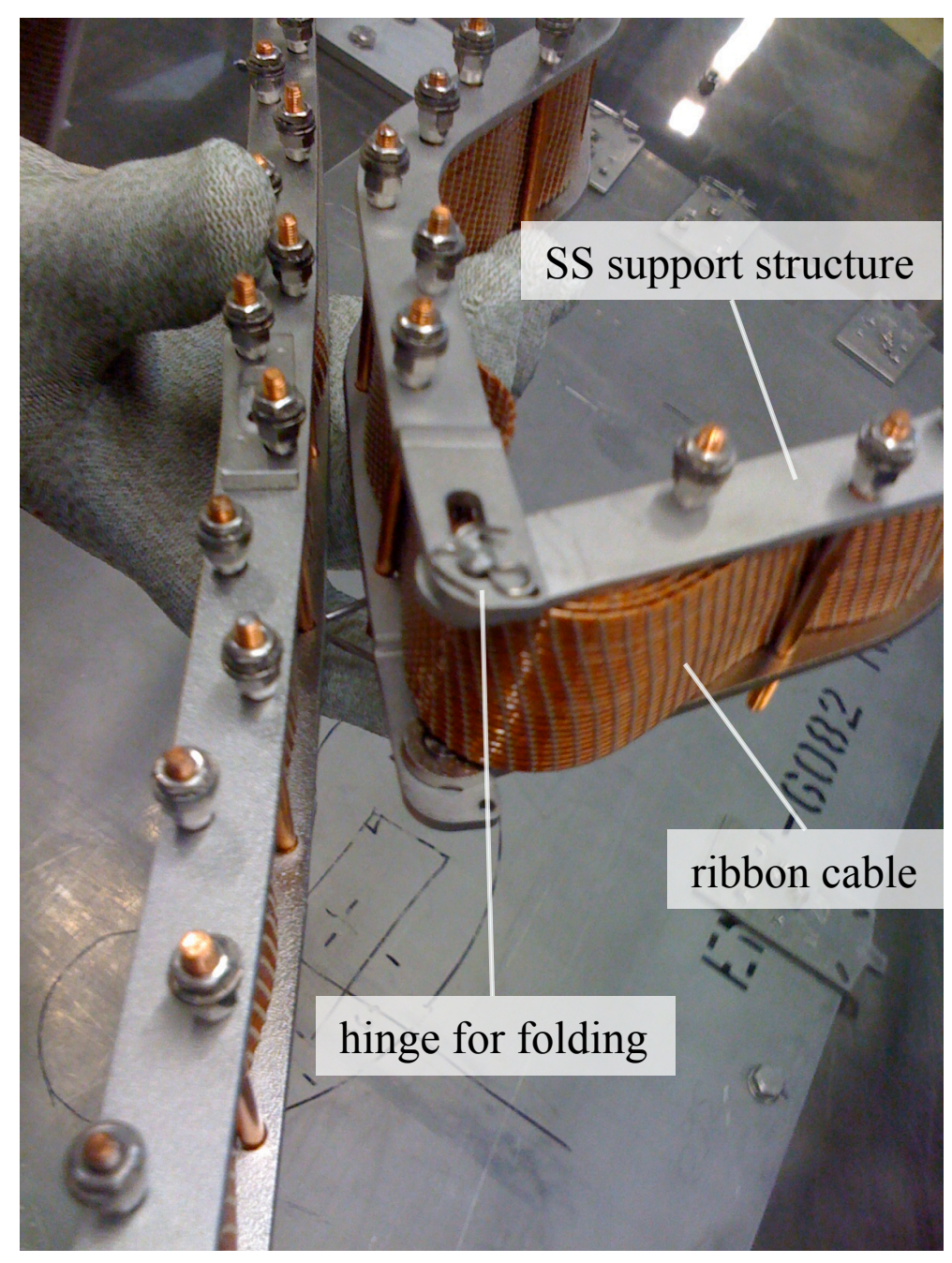

Figure 5: Support structure and four layers of 25-wire ribbon cable of a flat section of a $D L$ during a folding test. The side walls are from $2 \mathrm{~mm} S S$, connected by $3 \mathrm{~mm} S S$ rods with $\mathrm{Cu}$ bushes to keep the distance between the side walls. The polyimide-insulated wires are woven into ribbons by PEEK threads. The $0.2 \mathrm{~mm} \mathrm{Cu}$ sheets for ECR radiation protection are not yet installed in this photograph.

winding area for the CCs, the height of the side walls is $80 \mathrm{~mm}$ in these sections. The DLs in the TPs each consist of four such sections of similar length (denoted as CC sections in the following), connected by hinges, and one flat section. Each $\mathrm{CC}$ section contains a separate $\mathrm{CC}$ and, on the plasma side of the section, the main loop, which runs along all sections across the hinges.

The ECR radiation protection housing in the CC sections consists of a $0.1 \mathrm{~mm} \mathrm{SS}$ sheet with a $3 \mu \mathrm{m}$ PVD layer of $\mathrm{Cu}$ on the outer surface. The electric conductivity of this combination is sufficiently low to achieve the specified time resolution of the CCs (see section 2.1.2), whereas the microwave absorption of the $\mathrm{Cu}$ surface is expected to be significantly lower than of a steel surface (see section 3.2).

For both the flat and the CC sections, the ECR radiation protection sheets around the hinges for folding of the DLs were installed after the integration of the DLs into the $\mathrm{PV}$, whereas the remaining parts were completed with the sheets before folding the DLs. 


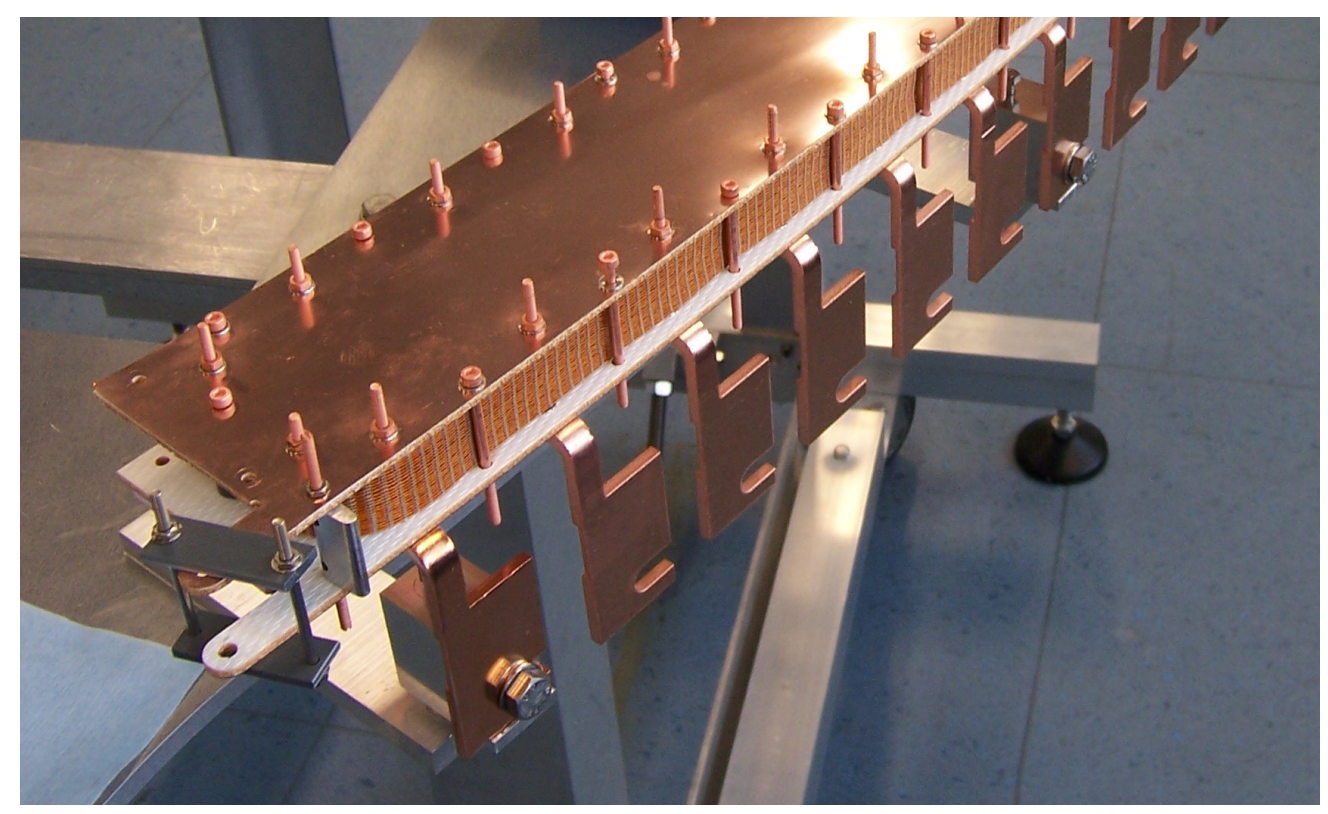

Figure 6: Assembly of a CC section of a DL. The side walls of $3 \mathrm{~mm}$ ceramic fibre reinforced ceramic compound (white) are covered on the outside by $0.1 \mathrm{~mm} S S$ sheets with a $3 \mu \mathrm{m}$ $\mathrm{Cu}$ layer. The two side walls are kept at a well-defined distance by PEEK separators (not visible) and are held together by the Cu-plated $3 \mathrm{~mm} S S$ rods, which at the same time define the position of the two layers of 25-wire ribbon cable forming the winding. The bores for the hinge which will connect to the next section are visible at the bottom left of the picture. The winding of the $C C$ is already installed, the winding of the main loop (one further layer of ribbon cable) will be installed once the sections of the DL are connected. Finally, the opening between the side walls will be closed by further $0.1 \mathrm{~mm}$ SS sheets with $\mathrm{Cu}$ layer. At the bottom of the section, the heat conduction rails/brackets of $4 \mathrm{~mm}$ $\mathrm{Cu}$ are visible, which will be used to attach the entire structure to the PV mechanically and thermally via $\mathrm{Cu}$ adapter blocks. The heat conduction rails/brackets on the top are not yet installed.

In fig. 7, we show a TP DL in fully folded and in unfolded state.

In both flat and CC sections, the ECR radiation protection sheets are overlapping. At the side walls, they are fixed by the nuts on the SS rods. On the upper and lower faces, the gaps are minimised by slightly bending the overlapping sections and using the resilience of the sheets (visible in fig. 9). We estimate that we could achieve gap widths below $0.2 \mathrm{~mm}$. Several mock-ups were tested in MISTRAL for sufficient microwave shielding to demonstrate the suitability of this design. One of the tests, with a full-scale prototype of a CC section, is described in section 4.1.3.2.

\subsubsection{Choice of materials and time resolution}

Since the flat sections of a DL are located very close to the PV, the additional eddy currents induced in the $2 \mathrm{~mm}$ SS side walls and in the $0.2 \mathrm{~mm} \mathrm{Cu}$ ECR radiation protection sheets are not significant, from the point of view of time resolution of the DL, as compared with the poloidal eddy currents which are anyway induced in the PV (see section 2.1.2). 


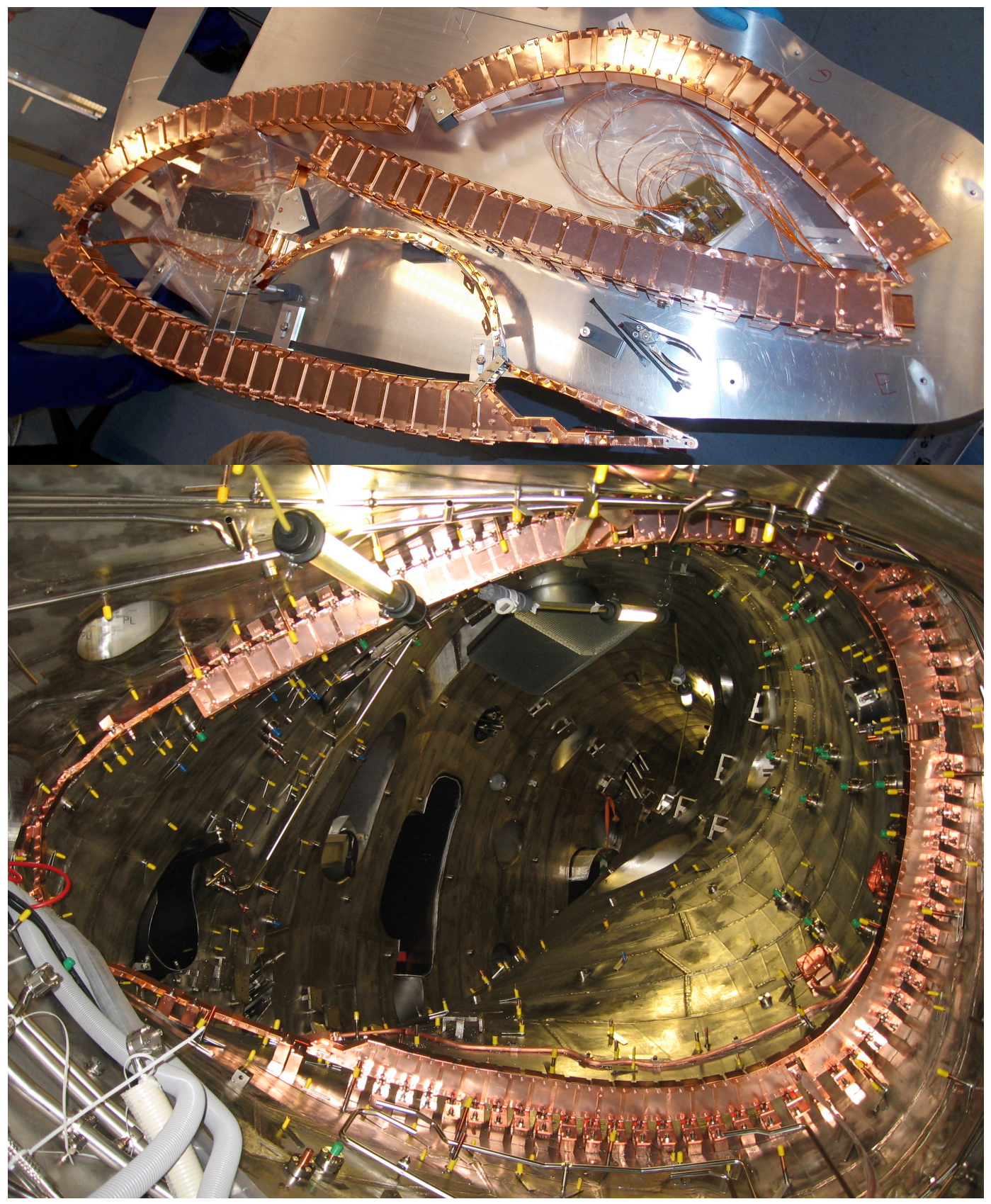

Figure 7: TP DL in fully folded state (top) and after integration into the PV (bottom). This loop consists of four CC sections and a short flat section, where the available space between $P V$ and WPEs is insufficient for a further CC. In the bottom photograph, the WPEs are not yet installed.

The time resolution of the CCs in this design would however also be limited to $\sim 10 \mathrm{~ms}$ instead of reaching the specified value of $1 \mathrm{~ms}$. In contrast, the choice of the ceramic compound provides sufficient rigidity, and the electric conductivity of the Cu-plated SS sheets is sufficiently low to limit eddy currents inductively coupling to the CC windings.

This is demonstrated by modelling the frequency dependence of the response of a CC and by measuring this response with the full-scale prototype in the MPTS (see section 3.2). To assess different options, windings with 100 turns and with 25 turns (the latter was 
realised in the full-scale prototype) with an impedance of $800 \Omega$ closing the circuit were considered in the simulation, each either

(1) without parasitic inductance, or

(2) with $4 \mathrm{~m} \Omega$ resistance in the parasitic circuit (corresponding to $2 \mathrm{~mm}$ SS side walls and $0.2 \mathrm{~mm} \mathrm{Cu}$ sheets), or

(3) with $100 \mathrm{~m} \Omega$ resistance in the parasitic circuit (corresponding to non-conducting side walls and $0.1 \mathrm{~mm}$ SS sheets with $3 \mu \mathrm{m} \mathrm{Cu}$ layer, as realised).

The coupling between the winding and the parasitic inductance was set to 0.66 (estimated from an analysis of the geometry). The measurements were taken with and without the ECR radiation protection sheets, corresponding to cases (3) and (1), and with an impedance of $\sim 1 \mathrm{k} \Omega$ closing the circuit. The results are shown in fig. 8. (The simulation

Absolute value (normalised)

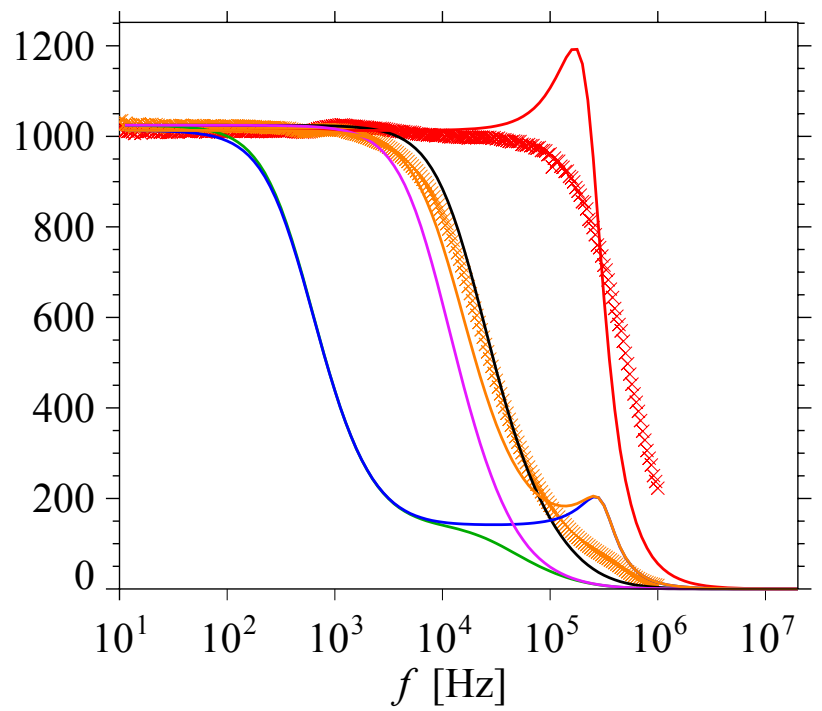

Phase of normalised data

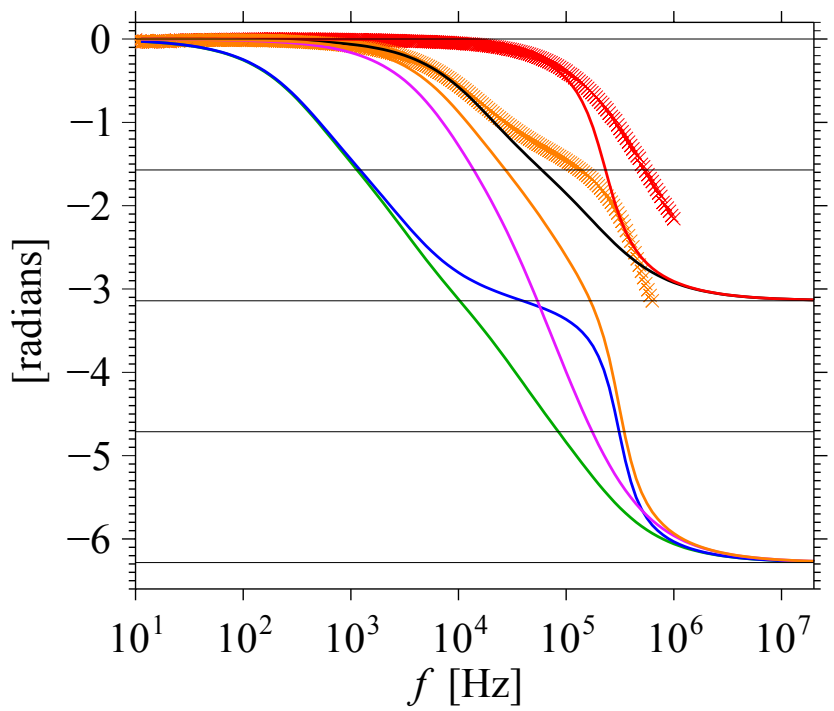

Figure 8: Simulation of the response of a $C C$ and comparison with the behaviour of the prototype measured in the MPTS. The response is normalised to the response of a rectangular flux loop with a single winding and very low self-inductance (experimental data, crosses) or to the theoretical response $\propto \omega$ of such a flux loop (simulation data, lines). The different simulation cases are: windings with 100 (black/green/violet) or 25 turns (red/blue/brown), without parasitic inductance (black/red), or with $4 \mathrm{~m} \Omega$ resistance in the parasitic circuit (green/blue), or with $100 \mathrm{~m} \Omega$ resistance in the parasitic circuit (violet/brown). For the measurements, the prototype was placed into the MPTS with (brown) and without (red) its ECR stray radiation protection.

and test frequency range is extended to above $10 \mathrm{MHz}$ for a better overview of the response, although the specification requires a test up to $1 \mathrm{kHz}$ only.)

Some deviations between simulation and measurements are visible, which may be due to the simplified geometry of the simulated current path of the parasitic inductance and to differences in the cable capacitance. Nevertheless, the characteristic frequencies, above which the response of the coil deviates from that of a simple flux loop, are well reproduced. 
The test results demonstrate that the $\mathrm{CC}$ signals can be used even significantly above the specified frequency of $1 \mathrm{kHz}$. In contrast, the simulation case (2) for $2 \mathrm{~mm}$ SS side walls and $0.2 \mathrm{~mm} \mathrm{Cu}$ sheets shows significant damping and phase shift already below $1 \mathrm{kHz}$ (green and blue curves in fig. 8).

\subsubsection{Dimensioning and thermal balance of final design}

4.1.3.1 Flat sections The maximum total thermal load expected to a DL flat section per length is $\sim 30 \mathrm{~W} / \mathrm{m}$ due to ECR stray radiation (assuming $50 \mathrm{~kW} / \mathrm{m}^{2}$ power density and an absorption of $0.6 \%$ ), plus $\sim 125 \mathrm{~W} / \mathrm{m}$ due to thermal radiation from the back faces of WPEs (with additional thermal $\mathrm{Cu}$ shields attached to the heat sinks, assuming a local thermal load of $500 \mathrm{~kW} / \mathrm{m}^{2}$ to the wall protection tiles and a thermal emissivity of 0.5 of $\mathrm{Cu}$, see section 3.3 ).

The flat sections are attached to the PV by $\mathrm{Cu}$ brackets of $2 \mathrm{~mm}$ thickness (see fig. 9). Neighbouring pairs of brackets have a typical separation of $0.2 \mathrm{~m}$ along the DL. Measurements on a mock-up of a DL flat section in a vacuum chamber with electric heater showed that the thermal conductance along the DL corresponds to that of the full cross-sectional area of the $0.2 \mathrm{~mm} \mathrm{Cu}$ ECR radiation protection sheets, although they are not continuous, but overlapping. In the same tests, the heat resistance between the DL and the PV through a pair of $\mathrm{Cu}$ brackets and adapter blocks with graphite interlayer was measured to be $\sim 2 \mathrm{~K} / \mathrm{W}$. The temperature difference between the middle position between two pairs of brackets and the position directly at the pair of brackets is then $\sim 80 \mathrm{~K}$ (assuming no cooling by radiation). The additional temperature difference due to the total heat flux of $\sim 30 \mathrm{~W}$ per pair of brackets is $\sim 60 \mathrm{~K}$ between the DL and the PV. With a PV temperature of $60^{\circ} \mathrm{C}$, this results in a maximum temperature of $200^{\circ} \mathrm{C}$ in the $\mathrm{DL}$ flat sections. Similar results were obtained in a finite-element simulation of the arrangement, which includes the cooling of the DL by thermal radiation. The simulations also show that the temperature limit of the winding wire is exceeded in long-pulse discharges if no thermal shields between the back faces of the graphite tiles and the DL are installed [40].

4.1.3.2 Sections with compensation coils Due to the larger height of the CC sections, the absorbed power per length from ECR stray radiation and also by thermal radiation from the back faces of WPEs is significantly larger than in the flat sections. We estimate $\sim 90 \mathrm{~W} / \mathrm{m}$ due to ECR stray radiation plus up to $\sim 150 \mathrm{~W} / \mathrm{m}$ due to thermal radiation from the back faces of WPEs (with additional thermal $\mathrm{Cu}$ sheets, same conditions as in section 4.1.3.1). In addition, due to the poorer heat conductivity of the $0.1 \mathrm{~mm}$ SS ECR radiation protection sheets, the parabolic temperature profile (neglecting cooling by emissison of thermal radiation) between two neighbouring brackets, which establish the thermal contact to the PV, will be much steeper than in the flat DL sections.

We chose a design with 20 heat conductors per metre of length of the DL on each of the two side walls, which continue as brackets to attach the DL to the PV mechanically and thermally (see fig. 10). $\mathrm{Cu}$ adapter blocks and graphite layers are used between the brackets and the PV, as discussed in section 3.4. Along the side walls, these $\mathrm{Cu}$ heat conductors have a rectangular cross-section of $10 \mathrm{~mm} \times 4 \mathrm{~mm}$. They thus reduce the effective electric resistance of the ECR radiation protection housing parallel to the winding 


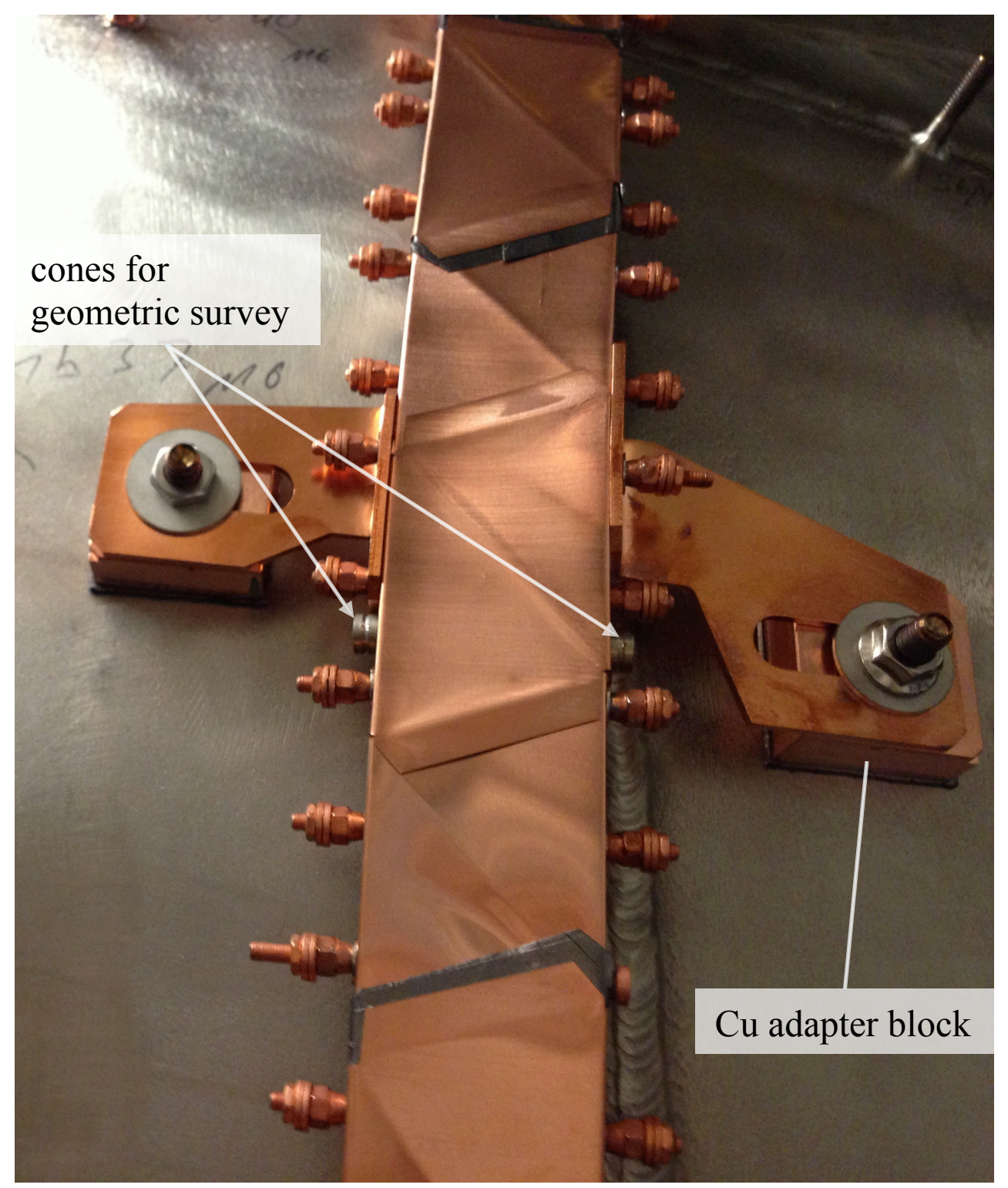

Figure 9: $D L B P$ (flat design) after integration into the $P V .2 \mathrm{~mm} \mathrm{Cu}$ brackets are used to connect the DL mechanically and thermally to the PV. Cu adapter blocks are placed between the brackets and the $P V$. The lower faces of these blocks are individually machined to fit to the surface of the $P V$. The remaining mismatch is balanced by graphite layers of $1 \mathrm{~mm}$ thickness between the adapter blocks and the plasma vessel (see section 3.4). The $3 \mathrm{~mm} S S$ rods placed along the $D L$ with their nuts and washers are $\mathrm{Cu}$ coated to reduce the microwave absorption and hence the thermal load to the DL. The entire DL is covered by overlapping $0.2 \mathrm{~mm} \mathrm{Cu}$ sheets to screen the ECR stray radiation. Graphite layers of $0.2 \mathrm{~mm}$ thickness are placed between some of theses sheets to assure a sufficiently high ohmic resistance along the loop, limiting the electromagnetic forces in case of a fast shutdown of the main magnetic field or of a fast plasma decay (see section 4.1.4.1).

by $20 \%$. This resistance is still sufficiently high, as was demonstrated by the prototype test in the MPTS, reported in section 4.1.2. In addition, $1 \mathrm{~mm} \mathrm{Cu}$ plates are attached to each pair of heat conductors as thermal shields (see fig. 11). These serve to directly 


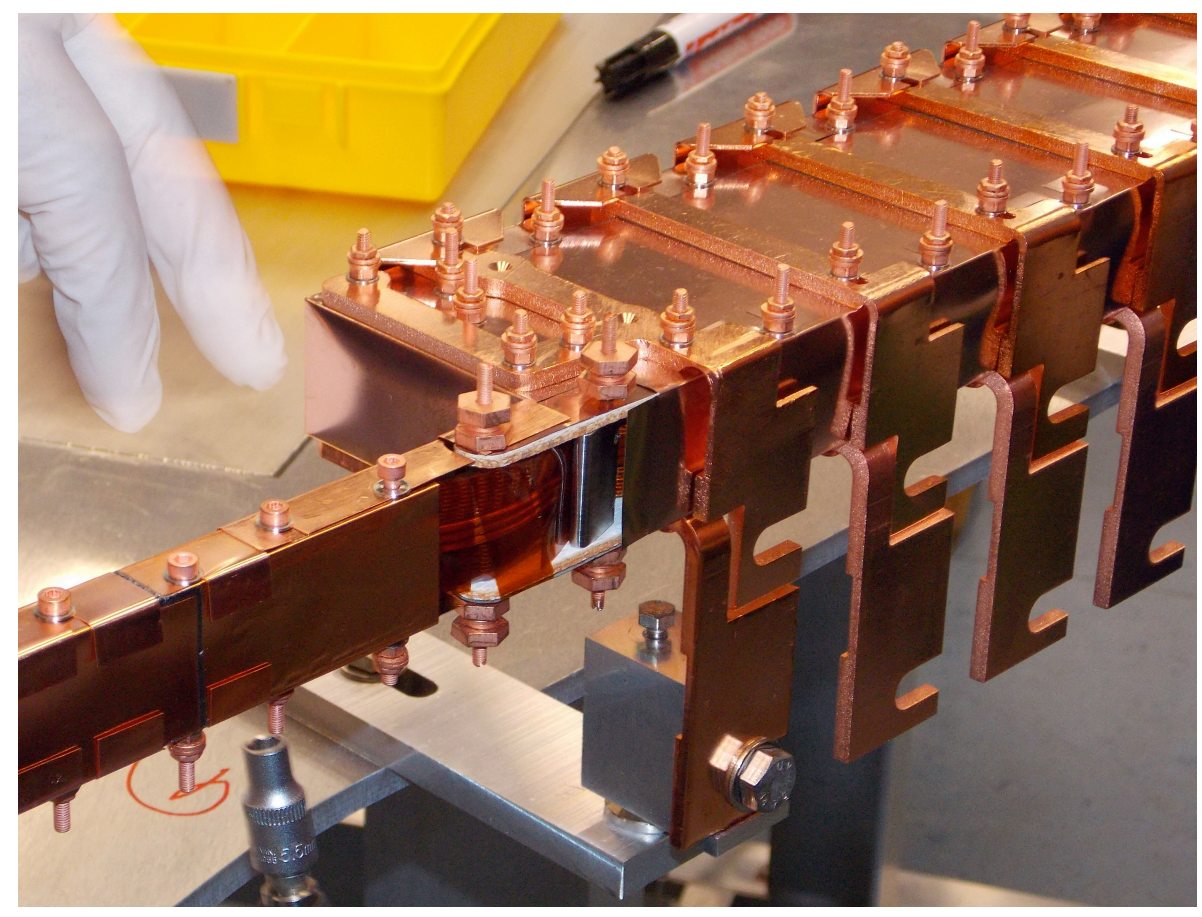

Figure 10: Transition between a CC section (right) and a flat section (left) of a DL, with a hinge between the two sections. The assembly is almost complete, except for the ECR radiation shielding in the hinge area, which is installed after the integration of the DL into the $P V$. The heat conductors of $10 \mathrm{~mm} \times 4 \mathrm{~mm} \mathrm{Cu}$ are in place, with a spacing of $\sim 50 \mathrm{~mm}$ along the DL. These conductors continue as brackets to fix the $\mathrm{DL}$ on $\mathrm{Cu}$ adapter blocks. Due to space restrictions, all brackets protrude on the same (here: lower) side of the DL.

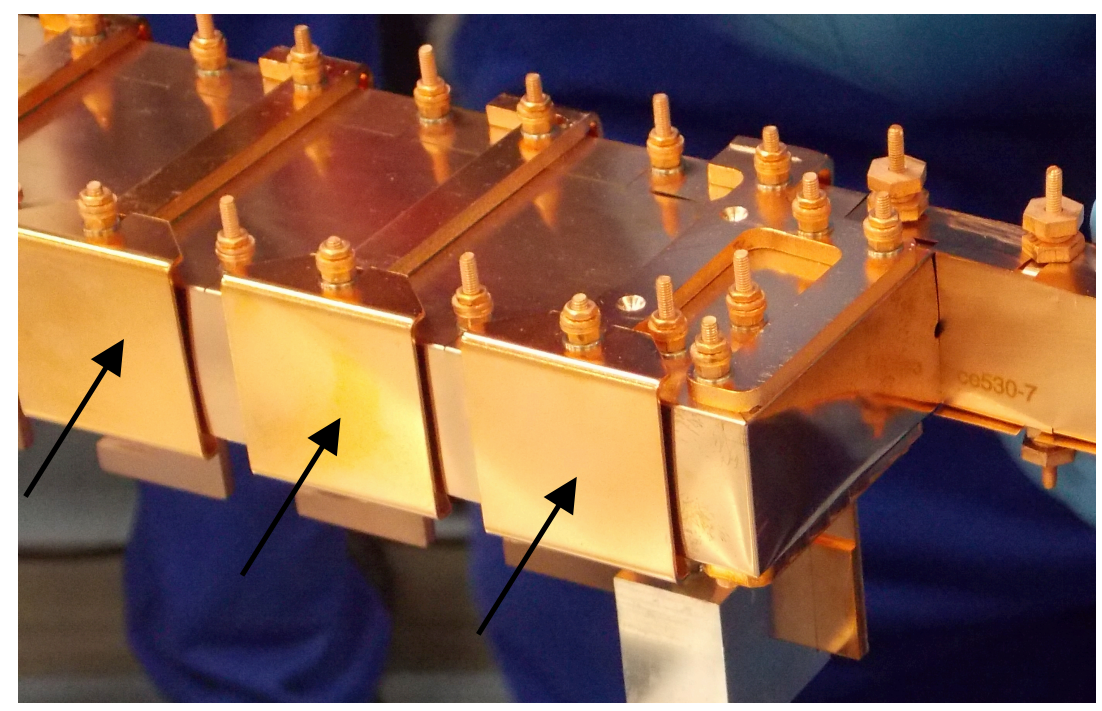

Figure 11: Thermal shields of $1 \mathrm{~mm} \mathrm{Cu}$ (indicated by arrows) on the WPE side of a CC section of a $\mathrm{DL}$. The $\mathrm{Cu}$ of $1 \mathrm{~mm}$ thickness conducts the heat irradiated from the back faces of the WPEs directly to the heat conductors, without loading the $0.1 \mathrm{~mm}$ SS ECR radiation protection sheets. On the heat conductor in contact with the rightmost thermal shield, two cones for geometrical surveys are visible. 
conduct to the heat conductors the heat which is radiated from the back faces of the WPEs to the DL. Otherwise, the $0.1 \mathrm{~mm}$ SS sheets with their poor thermal conductivity would also have to transmit this load. These additional $1 \mathrm{~mm} \mathrm{Cu}$ plates do not touch each other or the continuous SS sheets, in order to maintain the high ohmic resistance of the housing parallel to the winding.

With these dimensions, we estimate that, under stationary conditions, each heat conductor must transmit $\sim 2.5 \mathrm{~W}$ from the top face (toward the WPEs) plus $\sim 3 \mathrm{~W}$ from the side face plus $\sim 0.3 \mathrm{~W}$ from the bottom face (toward the PV) to the PV. The resulting temperature differences are $\sim 100-120 \mathrm{~K}$ between the top end of the heat conductor and the point of maximum temperature on the top ECR radiation protection sheet in the centre between two heat conductors, $\sim 30 \mathrm{~K}$ along the heat conductor to the adapter block and $\sim 30 \mathrm{~K}$ across the graphite layer to the PV. With a PV temperature of $60^{\circ} \mathrm{C}$, a maximum temperature of $220-240^{\circ} \mathrm{C}$ of the DL housing would result, if cooling by thermal radiation from the $\mathrm{DL}$ is neglected.

We used the CC prototype in MISTRAL to qualify this design under $\sim 50 \mathrm{~kW} / \mathrm{m}^{2} \mathrm{ECR}$ radiation (note that it proves difficult to determine this power density with an uncertainty below $20 \%$ ). The temperature of the prototype was measured by thermocouples in various locations (see fig. 12), and the average temperature of the winding wire was calculated

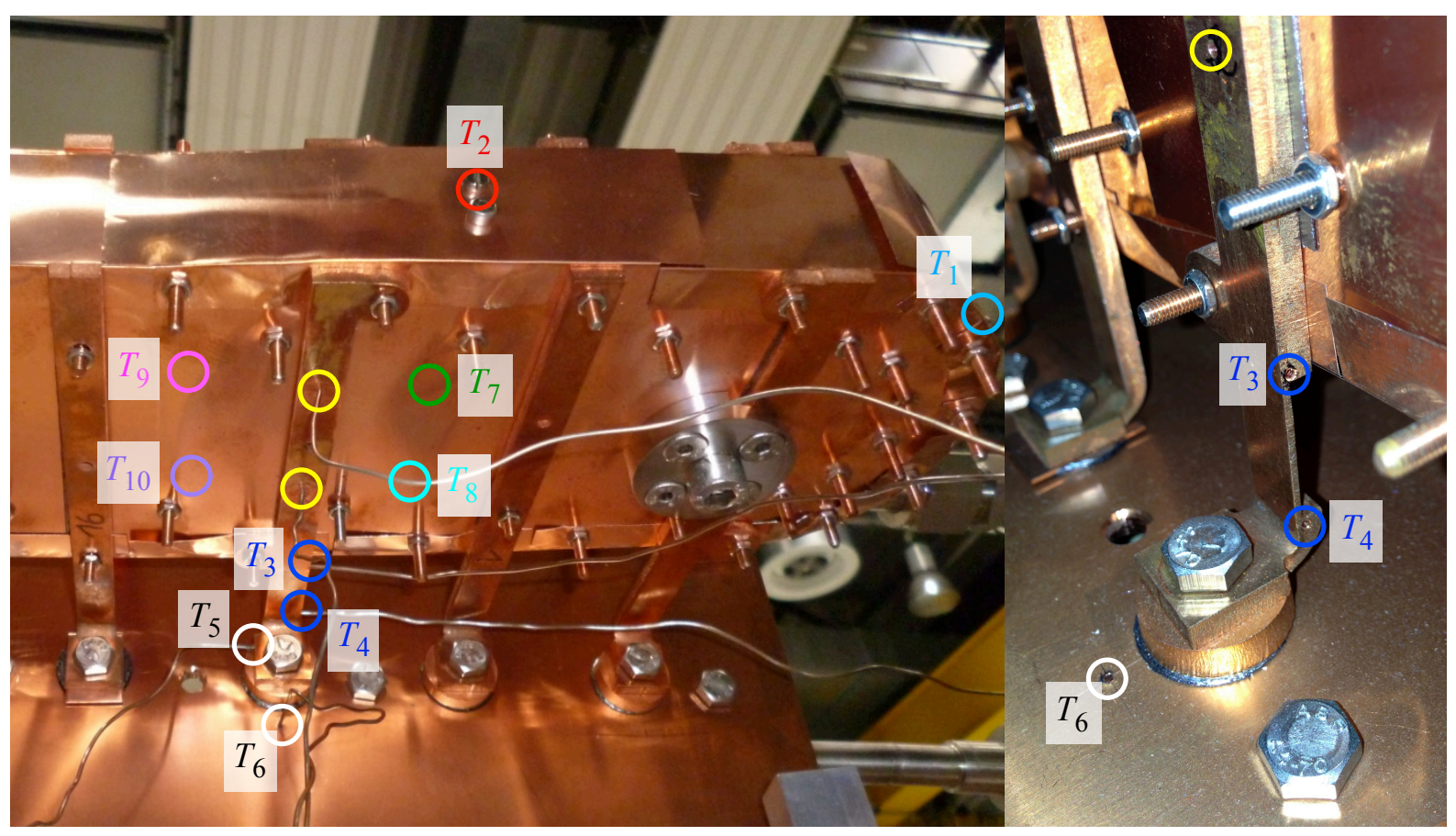

Figure 12: Positions of thermocouples on the CC prototype. The same colours as for the time traces in fig. 13 are used for the thermocouples measuring $T_{1}, T_{2}$ and $T_{7}-T_{10}$. On the right, the lower part of the heat conductor/bracket with reduced cross section is enlarged (without the thermocouples inserted into their respective bores). This section is used to determine the heat flux along this bracket by calculating the temperature difference $T_{3}-T_{4}$. From $T_{5}$ and $T_{6}$ the heat transmission between the bracket and the water-cooled $\mathrm{Cu}$ base plate is determined.

from its ohmic resistance during some of the test runs. In the prototype, two of the heat 
conductors were modified to obtain the heat flux through a known length with known cross-sectional area and heat conductivity (see fig. 12). The temperature evolution during a 30 min qualification run is shown in fig. 13. We note that the temperatures are not yet

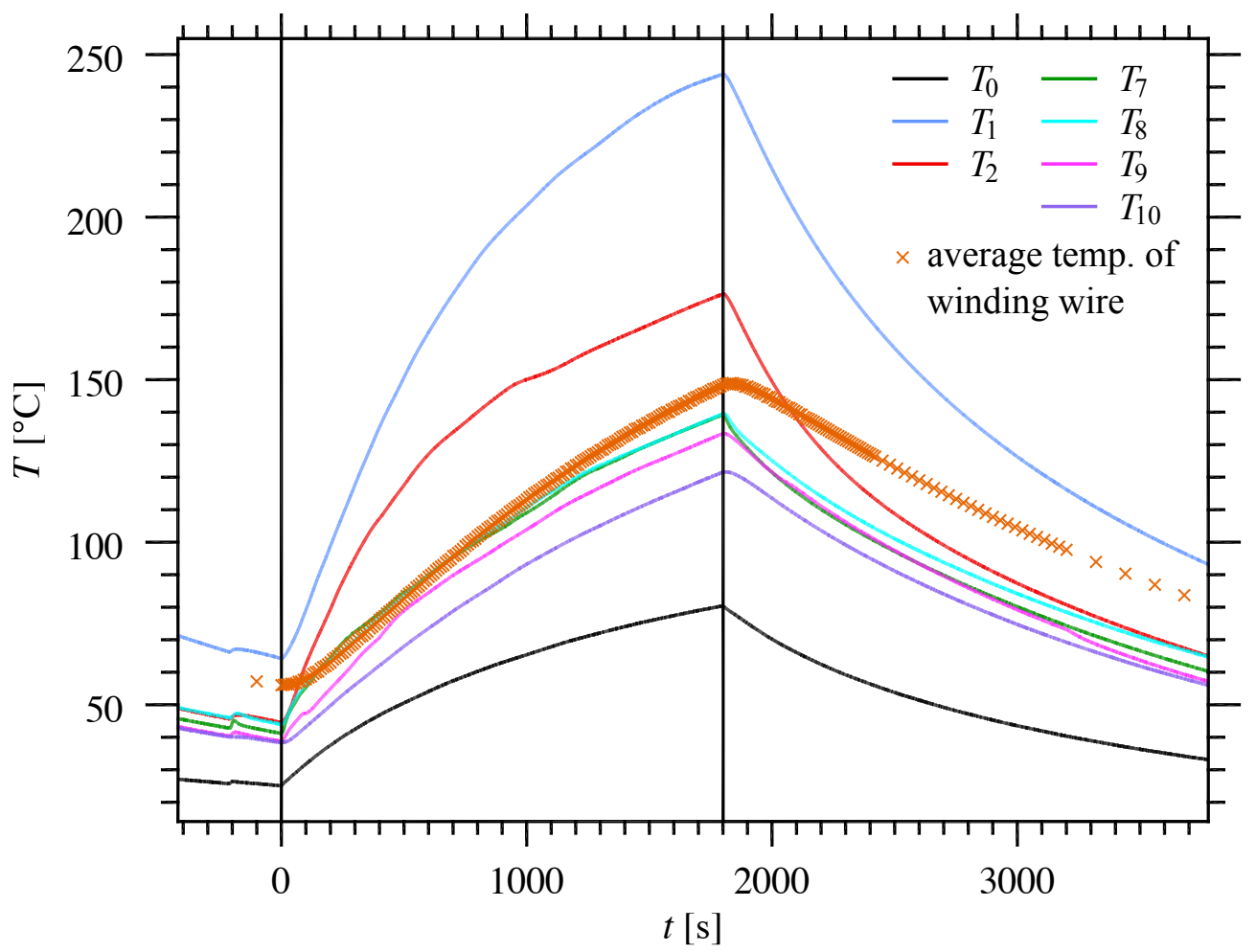

Figure 13: Test of the prototype of a DL CC section shown in fig. 12 in MISTRAL with a stray radiation power density of $\sim 45 \mathrm{~kW} / \mathrm{m}^{2}$. The microwave power was applied in the 30 min interval between the two vertical lines. The test starts at an elevated temperature due to preceding microwave pulses. Temperatures were measured by thermocouples in the positions corresponding to those indicated in fig. 12: $T_{0}$ : centre of the water-cooled $\mathrm{Cu}$ base plate; $T_{1}$ : rounded section at the end of the prototype, far from the next heat conductor; $T_{2}$ : behind the top sheets in the middle between two heat conductors; $T_{7}$, $\ldots, T_{10}$ : behind the side sheet in the middle between two heat conductors. The average temperature of the winding wire was calculated from its ohmic resistance.

stationary at the end of this interval. The highest temperature is measured as $T_{1}$, because the heat conduction path through $0.1 \mathrm{~mm}$ SS sheets to the next heat conductor is largest from this position. The final design was adapted, routing heat conductors close to the end faces of the CC sections (visible in fig. 11). The temperatures $T_{7}, \ldots, T_{10}$, measured between the ECR radiation protection sheets and the ceramic side walls in the middle between two heat conductors are all quite similar. The average winding temperature (brown crosses) shows reasonable values between the temperatures measured at the ECR radiation protection sheets.

From measurements of $T_{3}$ and $T_{4}$ in stationary phases of a different MISTRAL run and from the known heat conductance of the section of heat conductor between the corresponding measuring positions we calculated heat fluxes of $3.5-5.5 \mathrm{~W}$ at a microwave power density of $30 \mathrm{~kW} / \mathrm{m}^{2}$, and $\sim 7 \mathrm{~W}$ at $45 \mathrm{~kW} / \mathrm{m}^{2}$. The heat flux resistance across the 
interface $\mathrm{Cu}-\mathrm{Cu}$-graphite foil $(1 \mathrm{~mm})-\mathrm{Cu}$ is calculated from these heat fluxes and from $T_{5}$ and $T_{6}$ to be 1.0-1.2 K/W, corresponding to a heat transmission coefficient of 3.3$4 \mathrm{~kW} /\left(\mathrm{m}^{2} \mathrm{~K}\right)$.

If an equal partition of the heat flux to all heat conductors is assumed, we obtain microwave absorption coefficients of $1.5-2.5 \%$ for the heat fluxes of 3.5-5.5 W, significantly larger than expected for a smooth $\mathrm{Cu}$ surface (see section 3.2). Since the thermal layout of the design is already marginal (see section 4.1.3.2), temperature sensors (Pt1000) are installed in several critical locations in the CC sections as well as in the flat sections of all DLs to monitor the temperature of the ECR radiation protection sheets. Each Pt1000 sensor is clamped between an ECR radiation protection sheet and a flat $\mathrm{Cu}$ block screwed to the inner surface of the sheet. The location corresponds to that of the thermocouple measuring $T_{2}$ in the CC prototype, which was an upper bound on the average temperature of the winding (red trace versus brown crosses in fig. 13). If the readings of the temperature sensors should show that this is necessary, the maximum temperatures in the ECR stray radiation protection housing could be reduced by modifying the design of the DL's own $1 \mathrm{~mm} \mathrm{Cu}$ thermal shields (visible in fig. 11), such as to leave still smaller gaps between them for the thermal radiation from back faces of the WPEs to the stray radiation protection housing.

\subsubsection{Electromagnetic forces}

4.1.4.1 Flat sections Under certain conditions, e.g., a fast shutdown of the superconducting coil system, but also a fast change of the diamagnetic plasma energy, like at the end of a plasma discharge, poloidal eddy currents can be induced in the PV and hence also in a DL. With an ohmic resistance of $0.6 \mathrm{~m} \Omega / \mathrm{m}$, as calculated for a continuous ECR stray radiation shield with a cross-sectional area of $30 \mathrm{~mm}^{2}$, the resulting forces could deform the $\mathrm{Cu}$ brackets attaching the DL to the PV and tear off the segments of the stray radiation shield. Instead of changing the design significantly, we chose to reduce the forces by increasing the ohmic resistance along the DL. A reduction of the forces by a factor of 7 was required, i. e., the ohmic resistance along the DL must be at least $4 \mathrm{~m} \Omega / \mathrm{m}$. Measurements showed that this was already the case for most sections, presumably due to the interfaces between the individual shield segments. The condition must, however, be satisfied reliably for every poloidal section of the DL between brackets individually, since, otherwise, large currents could flow from the PV through the brackets and along the section with low resistance. In most sections between poloidally neighbouring brackets, we inserted an additional graphite layer of $0.2 \mathrm{~mm}$ thickness below one of the outer ECR stray radiation shield pairs (visible in fig. 9). This layer increases the ohmic resistance at the interfaces to the poloidally neighbouring shield segments. This way, the ohmic resistance along the DL could be increased to $>4 \mathrm{~m} \Omega / \mathrm{m}$ for all sections of more than $0.3 \mathrm{~m}$ poloidal length. For the remaining few sections, where this measure could not be realised, graphite layers of $0.2 \mathrm{~mm}$ thickness were inserted between the $\mathrm{Cu}$ brackets and the DL, such that the ohmic resistance for the current path between PV and DL was increased. By these measures, the electromagnetic forces to the DL flat sections due to eddy currents are reduced to a tolerable level. 
4.1.4.2 Sections with compensation coils The ohmic resistance to poloidal currents of a CC section is $\sim 10 \mathrm{~m} \Omega / \mathrm{m}$. A poloidal loop voltage of $0.4 \mathrm{~V} / \mathrm{m}$ will induce a current of $40 \mathrm{~A}$, exerting a force of $160 \mathrm{~N} / \mathrm{m}$ in a magnetic field of $4 \mathrm{~T}$. Due to the large number of $40 \mathrm{Cu}$ brackets attaching a length of $1 \mathrm{~m}$ to the plasma vessel, this electromagnetic force is of no concern.

\subsection{Expected signals}

The maximum plasma energy expected in W7-X is $5 \mathrm{MJ}$. The DLs were specified to measure the plasma energy with an accuracy of $10 \mathrm{~kJ}$. According to eq. (1), the maximum plasma energy corresponds to a change in the toroidal magnetic flux of $\Delta \Phi_{\text {dia, } \max } \approx$ $50 \mathrm{mWb}$, the specified measurement accuracy to a change in the toroidal magnetic flux of $\Delta \Phi_{\mathrm{dia}, \min } \approx 100 \mu \mathrm{Wb}$. If the CCs in the $\mathrm{TP}$ are to be used to distinguish if a flux change in the main loop is due to changes in the plasma energy or due to changes in the main field coil currents, they must be sufficiently accurate to measure changes in the magnetic field generated by the main field coils corresponding to $\Delta \Phi_{\text {dia, min }}$ in the main loop, i. e.,

$$
\Delta \Phi_{\text {comp., min }}=\Delta \Phi_{\text {dia, } \min } \frac{A_{\text {comp. }}}{A_{\text {main loop }}}
$$

where $A_{\text {comp. }}$ is the winding area spanned by the $\mathrm{CC}$ and $A_{\text {main loop }}$ is the winding area spanned by the main loop. Using 3 out of the 4 CCs in a TP, we obtain $\Delta \Phi_{\text {comp., min }} \approx$ $10 \mu \mathrm{Wb}$ as the required accuracy in each single $\mathrm{CC}$.

For the time scales of flux changes through the DLs and CCs, we can roughly distinguish between events slower or faster than the decay time of poloidal currents in the PV of $\sim 10-20 \mathrm{~ms}$. The slower events would be, e.g., a fast shutdown of the superconducting magnet system of $\mathrm{W} 7-\mathrm{X}(\sim 3 \mathrm{~s})$, the evolution of the plasma energy in a high-power, high-performance discharge $\left(W_{\max } / P_{\text {heat }} \sim 200 \mathrm{~ms}\right)$, or a very fast decay of the plasma energy within $\sim 10 \mathrm{~ms}$. An event on a faster time scale than $10 \mathrm{~ms}$ would be a loss of $10 \%$ of the plasma energy due to an ELM, like it was observed in tokamaks on a time scale of $\sim 100 \mu$ s for the main ELM event (see section 2.1.2).

For events changing the diamagnetic energy of the plasma on time scales $\gtrsim 10 \mathrm{~ms}$, essentially the full voltage signal due to the change $\Delta \Phi_{\text {dia }}$ will be induced in the DLs. In addition, voltages reflecting the ensuing eddy currents in the PV and in the superconducting magnet system will be induced in the main loops as well as in the CCs, in a ratio depending on their winding areas.

For events changing the diamagnetic energy on time scales $\lesssim 10$ ms, eddy currents will be induced in the PV, keeping the total toroidal flux within the PV constant. $\Delta \Phi_{\text {dia }}$ within the plasma will thus be balanced by a reverse fast change of the toroidal magnetic flux in the gap between plasma and PV. The voltage induced in the main loop therefore depends on the fraction of area between plasma and PV which its winding encompasses. The CCs only see the fast change in the toroidal flux between plasma and PV. To summarise, on the fast time scale $\lesssim 10 \mathrm{~ms}$, the flux change in a coil with winding area $A_{\text {coil }}$ is

$$
\Delta \Phi_{\text {coil }, \text { fast }}=\Delta \Phi_{\text {dia }}\left(\delta_{\text {enc }}-\frac{A_{\text {coil }}}{A_{\mathrm{PV}}}\right)
$$


where $A_{\mathrm{PV}}$ is the area within the PV in the plane of the coil, and $\delta_{\text {enc }}=1$ if the coil encloses the plasma and $\delta_{\text {enc }}=0$ if the coil does not enclose the plasma.

With these prerequisites, we obtain the maximum signal amplitudes and the required measurement accuracies to achieve $\Delta \Phi_{\text {dia, min }}$ and $\Delta \Phi_{\text {comp., min }}$ for the different winding numbers in discharges of $1800 \mathrm{~s}$ listed in table 2. The DLs BP are located very close to

\begin{tabular}{|c|c|c|c|c|c|c|}
\hline Coil & Event & $\begin{array}{l}\Delta \Phi_{\text {coil }} \\
{[\mathrm{mWb}]}\end{array}$ & $\begin{array}{l}\text { Time scale } \\
{[\mathrm{ms}]}\end{array}$ & $\begin{array}{l}\text { Number of } \\
\text { windings }\end{array}$ & $\begin{array}{l}\text { Maximum signal } \\
\text { amplitude }[\mathrm{V}]\end{array}$ & $\begin{array}{l}\text { Required } \\
\text { accuracy }[\mu \mathrm{V}]\end{array}$ \\
\hline \multirow{2}{*}{ DL BP } & \multirow{2}{*}{ (1) } & \multirow{2}{*}{50} & \multirow{2}{*}{10} & 50 & 250 & 2.8 \\
\hline & & & & $12 / 13$ & 65 & 0.7 \\
\hline \multirow{2}{*}{ DL TP } & \multirow{2}{*}{$(2)$} & \multirow{2}{*}{1} & \multirow{2}{*}{0.1} & 50 & 550 & 2.8 \\
\hline & & & & $12 / 13$ & 140 & 0.7 \\
\hline \multirow{2}{*}{ CCs TP } & \multirow{2}{*}{ (2) } & \multirow{2}{*}{$0.12-0.15$} & \multirow{2}{*}{0.1} & 100 & $120-150$ & 0.6 \\
\hline & & & & 25 & $30-40$ & 0.14 \\
\hline
\end{tabular}

Table 2: Maximum signal amplitudes for the DLs (main loops) and CCs and required measurement accuracy $U_{\min }$ of section 3.1.3 for the different winding numbers realised in the diamagnetic loops and compensation coils. Event (1) is a fast loss of the plasma energy of a high-power discharge of $5 \mathrm{MJ}$ within $10 \mathrm{~ms}$, event (2) is the loss of $10 \%$ of the plasma energy within $100 \mu$ s due to an ELM. For the DL TP, $A_{\text {coil }} / A_{P V} \sim 4 / 5$, therefore, according to eq. (2), only $1 / 5$ of the flux change due to this event is effective for this coil on the fast time scale. For the $D L B P, A_{\text {coil }} / A_{P V}$ is still closer to 1, therefore the loop voltage for event (2) on the fast time scale is smaller than for event (1) for this type of DL.

the $\mathrm{PV}$, such that $A_{\text {coil }} / A_{\mathrm{PV}} \approx 1$, and the signal due to a fast event is limited by the eddy currents in the PV.

The major potential source of perturbations for the signals of the DLs is their sensitivity to the main magnetic field. The magnetic flux due to this field of $3 \mathrm{~T}$ is $\sim 6.5 \mathrm{~Wb}$ for the BP DL, 5.3 Wb for the TP DL and 160-200 $\mathrm{mWb}$ for the CCs, hence larger by a factor of $\sim 100$ than $\Delta \Phi_{\text {dia, max }}$ and larger by a factor $\sim 5 \cdot 10^{4}$ than $\Delta \Phi_{\text {dia, min }}$. To maintain the specified accuracy of $10 \mathrm{~kJ}$ in the measurement of the diamagnetic energy without CCs (e.g., in the BP) therefore implies a measurement accuracy of the non-planar field coil currents of $\sim 0.3 \mathrm{~A}$. Even if the field coil currents are measured with this accuracy (e. g., by additional Rogowski coils around the field coil current feeds), even small changes in the coupling between field coils and DLs will perturb the measurements. These could be due to shifts and deformations of the field coils under electromagnetic forces, or of the windings of the DLs: A radial shift of $10 \mathrm{~cm}$ winding length by $0.3 \mathrm{~mm}$ already causes a perturbation corresponding to $\Delta \Phi_{\text {dia, min }}$.

The thermal load to the in-vessel magnetic diagnostics may prove particularly critical in this respect, as a thermal expansion of a DL within the toroidal flux of the main magnetic field will significantly change the flux through its winding. For long-pulse discharges, it may therefore prove necessary to use DLs with temperature control. Since the temperature of the entire PV may increase during a long-pulse discharge, it would even be favourable if actively cooled DLs or DLs outside the PV were not attached to the PV. An interesting option would be the use of the voltages induced in the superconducting 
coil circuits to correct the signals of the DLs on a slow time scale, as the superconducting coils certainly are "diamagnetic loops" under very strict temperature control. Even the use of $\mathrm{Cu}$ toroidal field coils to measure the diamagnetic energy was already demonstrated [45].

Significant perturbations may also be caused by poloidal currents between different divertor modules (e.g., due to asymmetries in the thermal load of the divertor modules). Such a current of $1 \mathrm{kA}$ (which is a maximum estimate) flowing between upper and lower divertor of one half module close to a DL either only on the torus outboard or only on the torus inboard side would cause a flux through the DL of $\sim 15 \cdot \Delta \Phi_{\text {dia, min }}$.

\section{Saddle coils}

\subsection{Design}

The SCs are placed directly outside the PV, in the cryovacuum, but on the "warm side" of the thermal shield. They are therefore compatible with the requirements of the cryovacuum and with temperatures between $-200^{\circ} \mathrm{C}$ and $+200^{\circ} \mathrm{C}$ (for the case of baking of the PV). Together with their signal cables up to the vacuum feedthrough, they are no longer accessible during the lifetime of W7-X after installation of the thermal shield. Therefore, and because of potential thermovoltages, plug connectors should be avoided in the design.

Each SC consists of an SS jacket with $3 \mathrm{~mm}$ outer diametre and 6 inner wires, which are embedded in a magnesium oxide insulation. The signal cable consists of two separate coaxial lines with single wire, $\mathrm{MgO}$ insulation and steel jacket. The two coaxial cables are twisted around each other. Each of them is terminatd by a brazed connector at the vacuum feedthrough. The six wires of the coil are connected in series and to the two wires of the signal cable within a T-shaped connector. In several of the coils, steel wires are used for the winding and the signal cables, in most of the coils, $\mathrm{Cu}$ wires are used. Each coil together with the T-shaped connector, the signal cable and the brazed connectors was delivered as a vacuum-tight sealed unit by the company Thermocoax.

Because of their small thickness, the SCs fit between the cooling pipes on the outside of the PV and the thermal shield (see fig. 2). They are attached to the plasma vessel by stud bolts. After installation of each SC, the as-built routing was surveyed by laser tracker.

\subsection{Expected signals}

The maximum magnetic flux due to plasma currents through one of the SCs adjacent to the TP (SHS = small half sector) is expected to be $\sim 17 \mathrm{mWb}$ at a plasma energy of $5 \mathrm{MJ}$ $(\langle\beta\rangle=5 \%$ at $2.5 \mathrm{~T}$ on the plasma axis, see fig. 14). The maximum magnetic flux through one of the larger SCs adjacent to the BP (LHS = large half sector) is 3-4 times larger, i. e., $\sim 60 \mathrm{mWb}$.

The PS currents measured by the SCs are driven by the plasma pressure. They therefore change on the same time scale as the plasma energy. The largest signal amplitude 


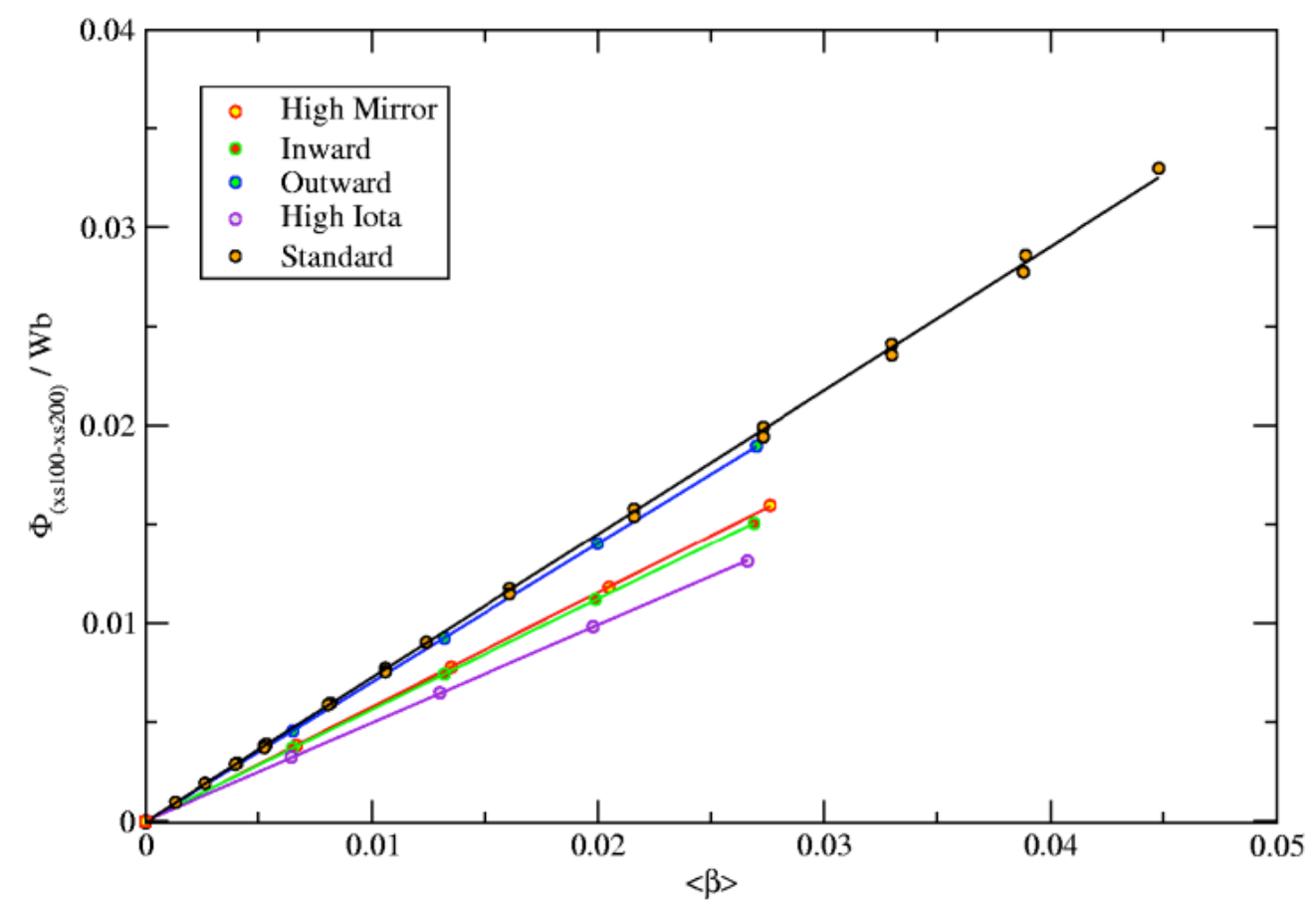

Figure 14: Simulated magnetic flux through SCs for 5 magnetic configurations. The signal shown is the sum of the top and bottom coil signals of a small half sector. The measured signal will be 6 times larger due to the number of windings in each coil.

to be accounted for would occur if the confinement time in a high-performance discharge with $\langle\beta\rangle=5 \%$ would suddenly deteriorate to $\sim 10 \mathrm{~ms}$. Since the SCs are located outside the PV, the longer time scale for toroidal currents in the PV of $\sim 25 \mathrm{~ms}$ applies (see section 2.2), and a loop voltage of $0.7 \mathrm{~V}$ for the SHS SCs (signal amplitude: $4 \mathrm{~V}$ ) and $2.5 \mathrm{~V}$ for the LHS SCs (signal amplitude: $15 \mathrm{~V}$ ) results. For an accuracy of $1 \%$ of the maximum magnetic flux in a half-hour discharge, the signal amplitude must be measured with an accuracy of $100 \mathrm{nV}$ for the SHS and $330 \mathrm{nV}$ for the LHS SCs.

The 7 circuits of the superconducting field coils, the control and trim coil circuits couple differently to the SCs. For the respective superconducting field coil circuits with strongest coupling, a variation of $1 \%$ of the nominal current will change the flux through the SHS SCs by $\sim 8 \mathrm{mWb}$, through the LHS SCs by $\sim 20 \mathrm{mWb}$. A variation of the control and trim coil currents by their maximum values will change the flux through the LHS SCs by 7-8 mWb. The coupling of these field coils with the SHS SCs is weaker.

The paths of the SCs are, as far as possible, designed in such a way that net plasma currents only generate a small magnetic flux through the SCs. This could be achieved to a high degree for the SHS SCs, whereas for the LHS SCs, net plasma currents do have an impact (see fig. 15).

Thermal currents flowing in the PV toroidally between different divertor modules could generate additional magnetic flux through the SCs. In a worst-case scenario, a $1 \mathrm{kA}$ current flowing either on the torus outboard or on the inboard side close to the path of an SC could generate a flux of almost $4 \mathrm{mWb}$ through this coil. The comparison of RC signals from inside and outside the PV (see section 2.2) will provide an indication of such PV currents. 


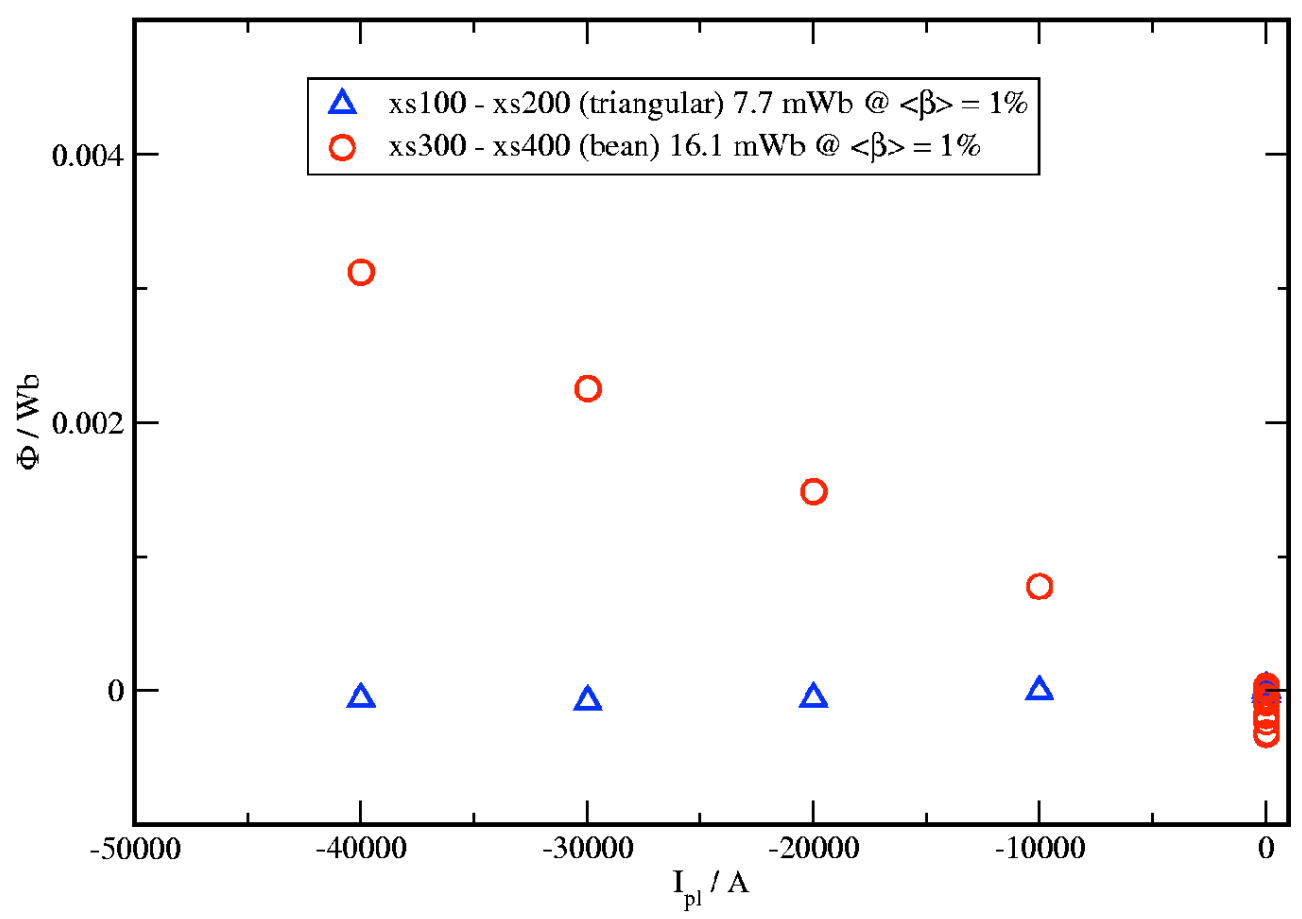

Figure 15: Simulated magnetic flux through the TP SCs (small half sector) and through the BP SCs (large half sector) for different values of the net plasma current (the sum of the fluxes through the top and bottom coils is shown). The simulation was done for 0 plasma pressure. For comparison, the fluxes for $\langle\beta\rangle=1 \%$ and 0 plasma current are written in the box.

\section{Rogowski coils (including segmented Rogowski coils)}

\subsection{Design and testing}

\subsubsection{Winding and basic design}

The RCs are the only magnetic sensors in W7-X located in pairs inside and outside the $\mathrm{PV}$, in order to distinguish between toroidal currents in the plasma and in the PV wall. A challenge for the design was the very narrow space both between the outer surface of the PV and the thermal insulation and between the inner surface of the PV and the WPEs, together with the requirement of sufficient winding area in the solenoid, the strong shaping of the PV and the requirement to find a closed poloidal path between port openings and cooling pipes on the outer PV surface.

We finally chose SS tubes with an outer diametre of $5 \mathrm{~mm}$ and a wall thickness of $1 \mathrm{~mm}$. Two layers of polyimide-insulated $\mathrm{Cu}$ wire are wound on the surface of these tubes. This is achieved quickly and uniformly on a lathe, while the tubes are still straight. After that, the tubes are bent into shape on a wooden model. For a sufficient winding area, several tubes are arranged in parallel on the PV surface and connected in series electrically. For practical reasons, it is not viable to cover the entire poloidal perimeter with a single length of these tubes. They are therefore subdivided into lengths between $300 \mathrm{~mm}$ and $1100 \mathrm{~mm}$. A set of 10 (in some cases 9) parallel tubes is called a "segment" and is attached to the $\mathrm{PV}$ by $\mathrm{SS}$ or $\mathrm{Cu}$ holders. 
On the outside of the PV, these segments are located in gaps between the cooling pipes (see fig. 2). For the CRCs, the gaps between adjacent segments must be minimised. In the design used for the outside of the PV, these gaps have a length of $20 \mathrm{~mm}$ where these "regular" segments are directly adjacent to each other. In some locations, however, a cooling pipe must be crossed. Here, even the height of the regular design exceeds the available space between cooling pipe and thermal insulation. "Crossover segments" are used for these short sections, resorting to the idea of specially machined Vespel ${ }^{\circledR}$ coil formers, resulting in a further reduced height but maintaining the same winding area density (see fig. 16).

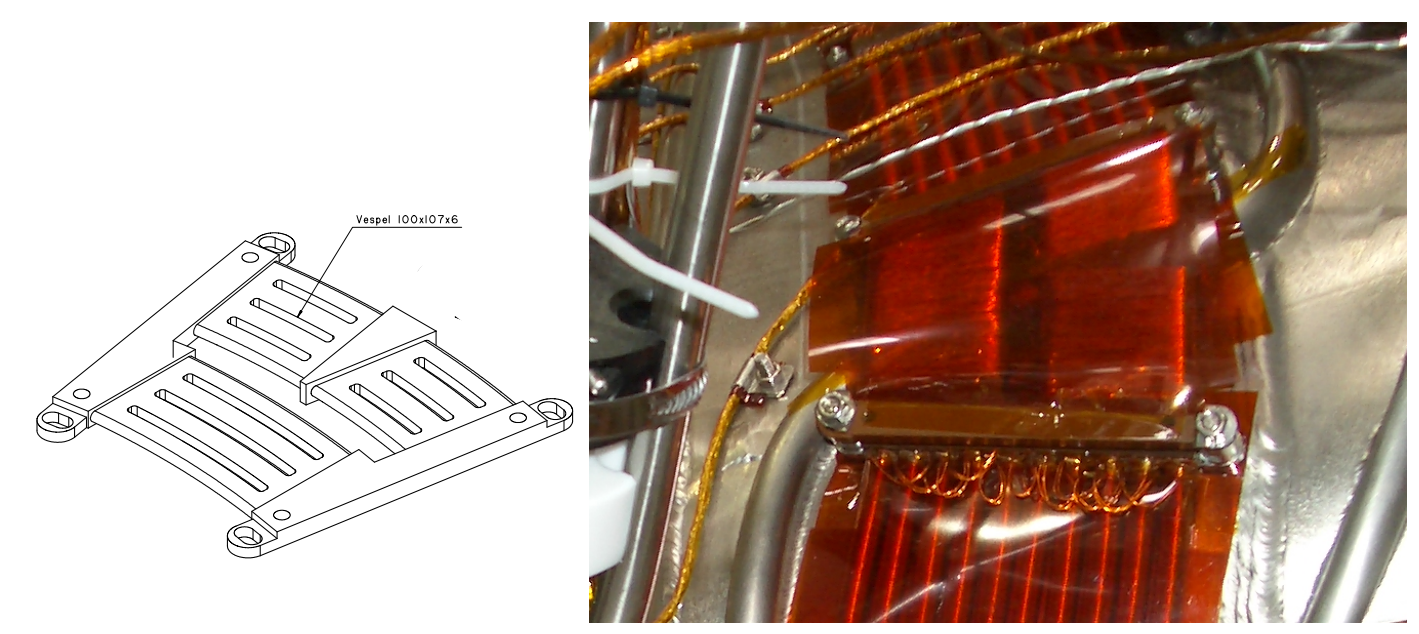

Figure 16: Crossover segment over PV cooling pipe: Drawing of the coil former and photograph of the installed segment. In this case, a bend in two dimensions had to be realised within the crossover segment in order to stay within the allotted space.

The solenoids of the in-vessel segments were inserted into SS tubes for ECR radiation shielding before they were bent into their CAD shape. The winding is separated from the shielding tubes by a glass fibre mesh for mechanical protection. The end holders of the segments are integrated into a $\mathrm{Cu}$ housing, such that the crimped connections between winding wires and signal cables are also shielded (see fig. 17). The signal cables are running in $\mathrm{Cu}$ tubes (see section 8), which fit into the end holders. For pumping, the shielding tubes and end holders contain holes with sizes below $1 \mathrm{~mm}$, less than half the wavelength of the ECR radiation. After calculations and tests of the response of the solenoid shielded by different tubes (see section 6.1.2) and after bending tests, an SS tube with $8.8 \mathrm{~mm}$ outer diametre and $0.25 \mathrm{~mm}$ wall thickness was chosen for shielding.

For each single Rogowski solenoid, the uniformity of the winding area density was measured by sliding an exciter coil, operated at $10 \mathrm{kHz}$, along the tube and recording the signal induced in the solenoid. Solenoids with values outside a narrow range (deviation from uniformity above $\sim 1 \%$ ) were rejected before bending. The same measurement was repeated after bending. For none of the solenoids, the bending did significantly change the local winding area density of the solenoids, i. e., the individual pattern of deviations from uniformity remained unchanged (to an estimated accuracy of $0.2 \%$ ).

The thermal contact between the in-vessel RC segments and the PV is realised at the end and intermediate holders through $\mathrm{Cu}$ adapter blocks and graphite interlayers, as 


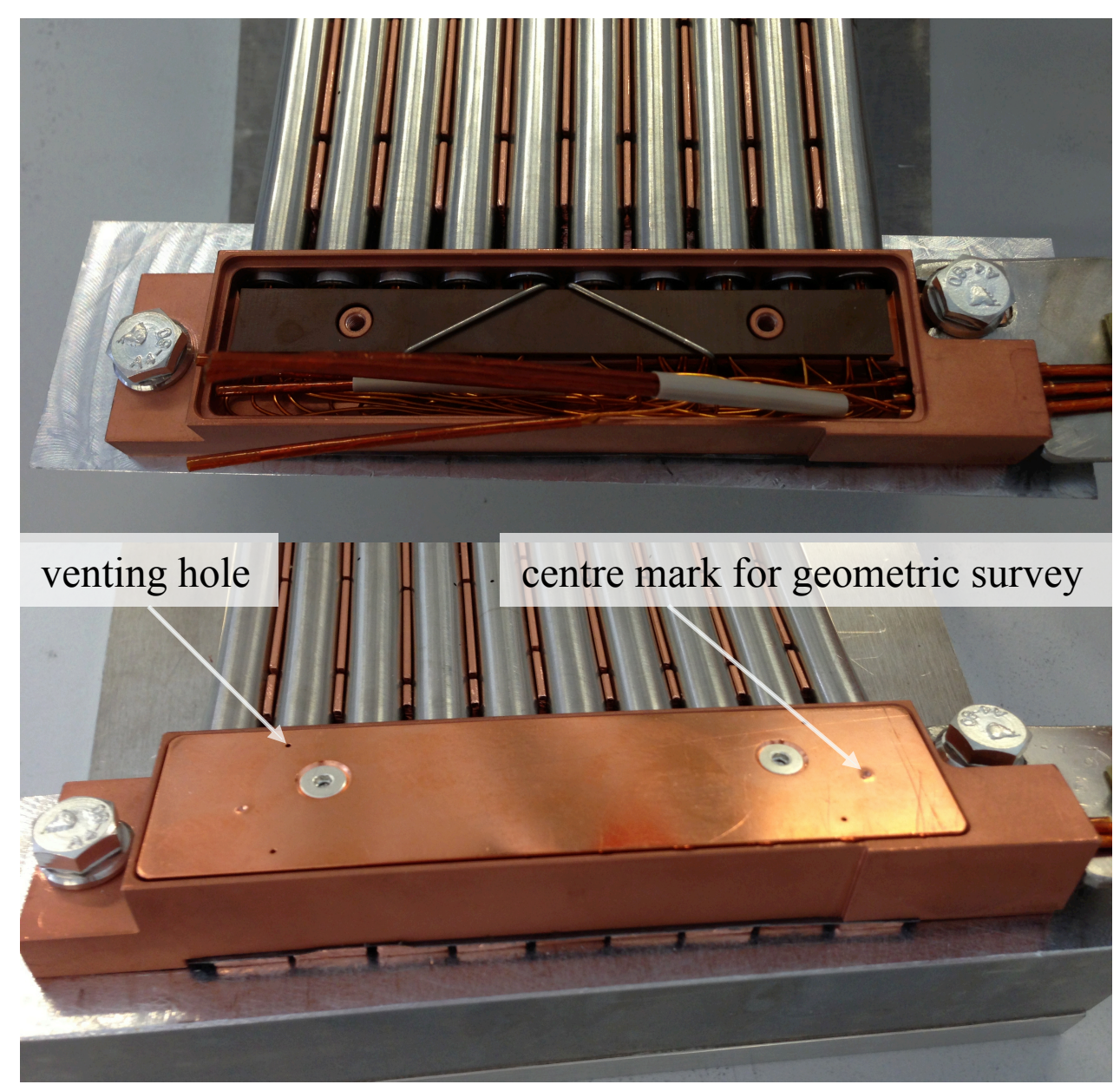

Figure 17: In-vessel RC end holder - open (top) and closed (bottom). The lower part of the end holder is machined from a $\mathrm{Cu}$ block. The SS shielding tubes of the solenoids fit tightly through holes in the end holder, the core tubes are fixed in the bar below the Vespel ${ }^{\circledR}$ sheet covering the crimp connections between the solenoids. The lower parts of the $\mathrm{Cu}$ heat conduction rails are clamped between end holder and $\mathrm{Cu}$ adapter block (in the above photographs, a temporary support replaces the adapter block). On the right, the signal cables are leaving the end holder (unshielded in these photographs). After integration, they will run in a $\mathrm{Cu}$ tube fitting into the block on the right hand side of the end holder. The lid has four venting holes and two centred marks with well defined positions for the geometric survey after integration.

described in section 3.4.

To improve the thermal conductance along the in-vessel solenoids, $\mathrm{Cu}$ profiles are inserted between neighbouring shielding tubes. These profiles are pressed directly onto the adapter blocks by the end and intermediate holders. The $\mathrm{Cu}$ profiles with their high electric conductivity are aligned perpendicular to the electric field induced in the solenoids, such that the induced signal is only moderately affected. Between the end and intermediate holders, the package of solenoids is pressed together and to the $\mathrm{Cu}$ profiles by SS bands point-welded around the package (see fig. 18).

Each in-vessel segment was pre-assembled on a purpose-built rig (see fig. 19) before being installed in the PV. 


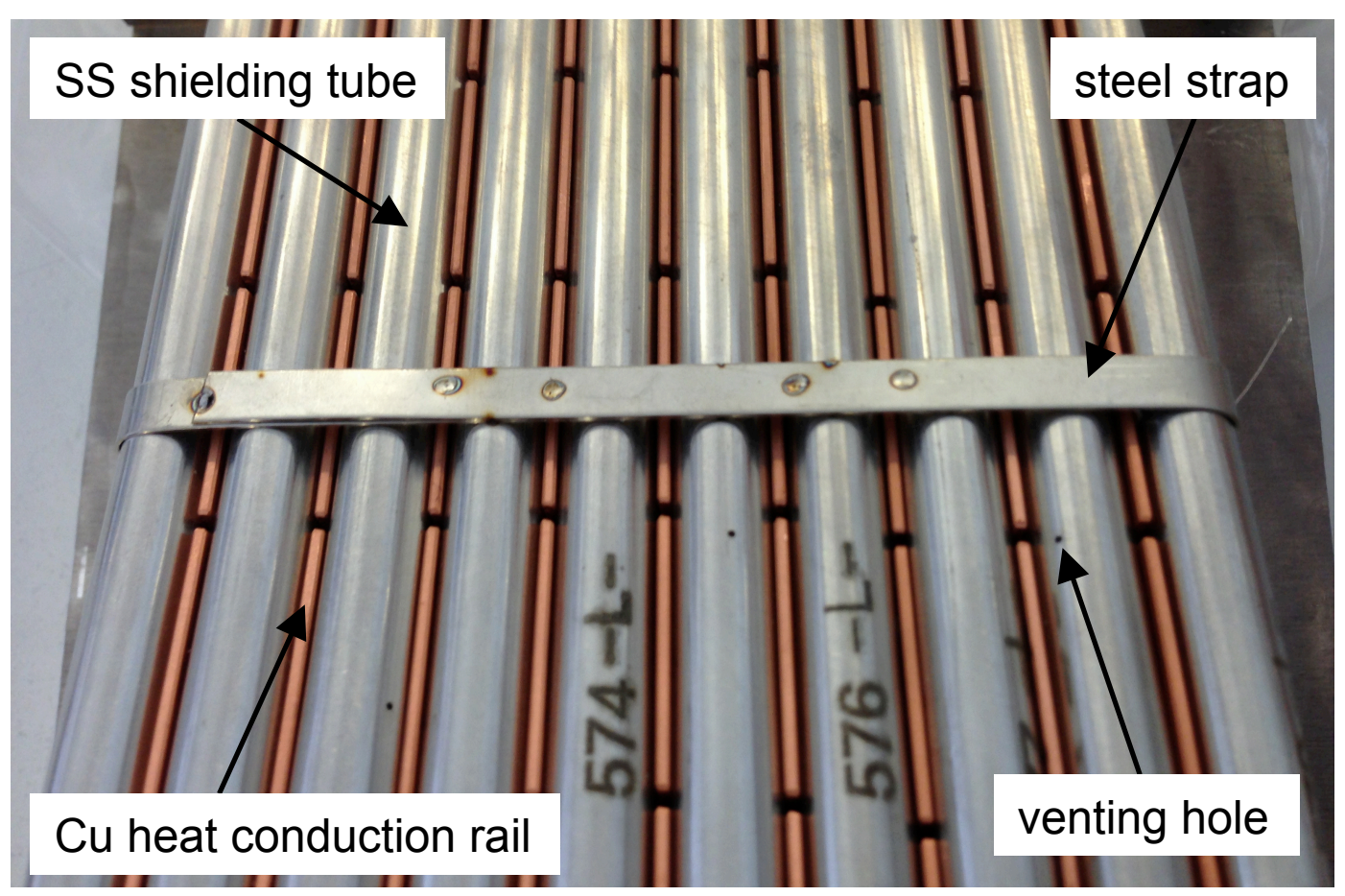

Figure 18: The solenoids and heat conduction rails of a Rogowski segment are held together tightly by steel straps in between the end and intermediate holders, such that a large contact area with good thermal contact between the shielding tubes and the heat conduction rails is maintained.

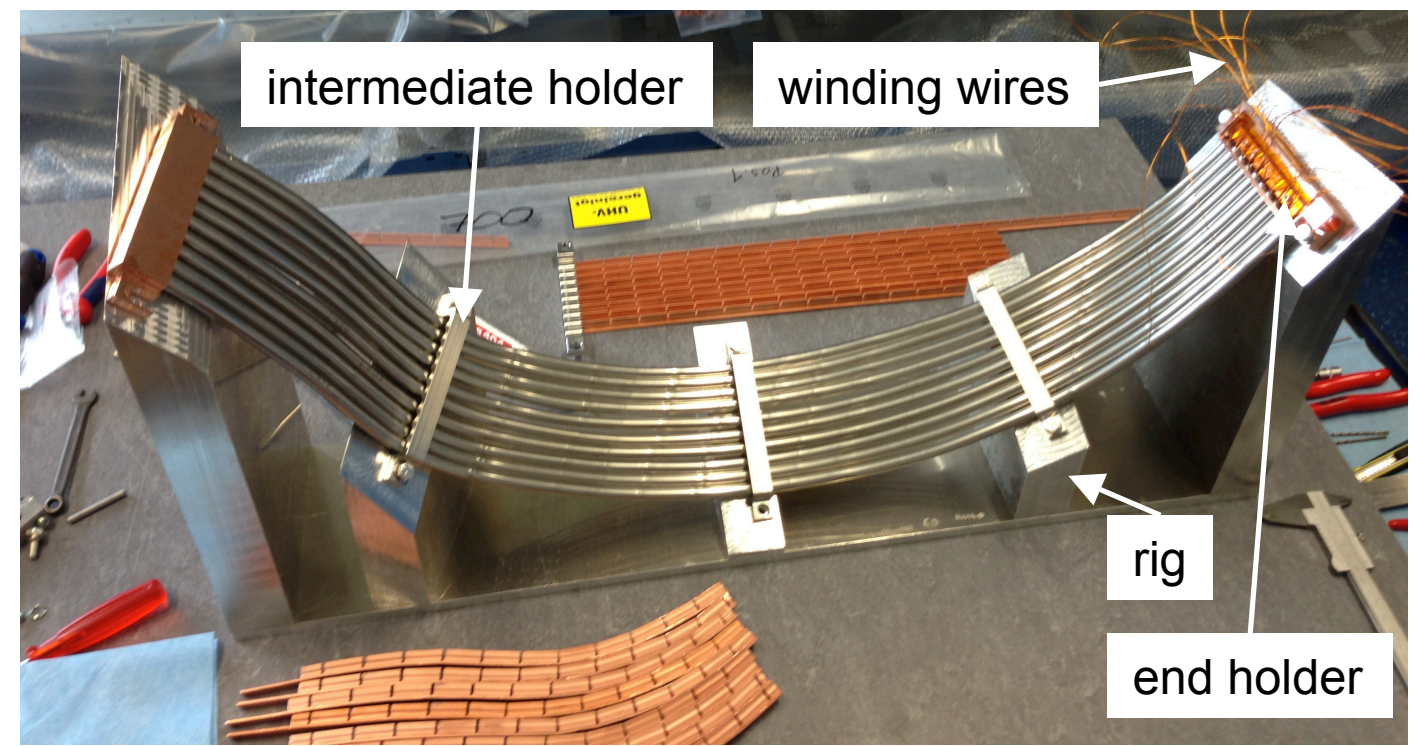

Figure 19: Assembly of an in-vessel RC coil segment on a purpose-built rig. The surfaces of the supports at the positions of the end and intermediate holders correspond to the surfaces of the $\mathrm{Cu}$ adapter blocks to which the segment will be mounted in the PV. The $\mathrm{Cu}$ heat conduction rails are ready for insertion (lower left, see fig. 21).

8-11 segments are required to cover the full poloidal perimeter. For redundancy, each segment is split electrically into two half segments, i. e., 4 or 5 of the solenoids are connected in series. For the CRCs, adjacent half segments are again connected in series, 
such that only two pairs of signal wires will connect the entire arrangement. For the SRCs, the signal of each half segment will be recorded separately. The design and first tests of the RCs outside the PV are treated in more detail in [46].

\subsubsection{Choice of materials and time resolution}

The response of a single RC solenoid of $470 \mathrm{~mm}$ length was tested in the MPTS (see section 3.2) without an outer shielding tube, with SS shielding tubes of $0.1 \mathrm{~mm}$ and $0.5 \mathrm{~mm}$ wall thickness and with a $\mathrm{Cu}$ shielding tube of $0.5 \mathrm{~mm}$ wall thickness. The results are shown in fig. 20, normalised to the signals of a flux loop of very low self-inductance.
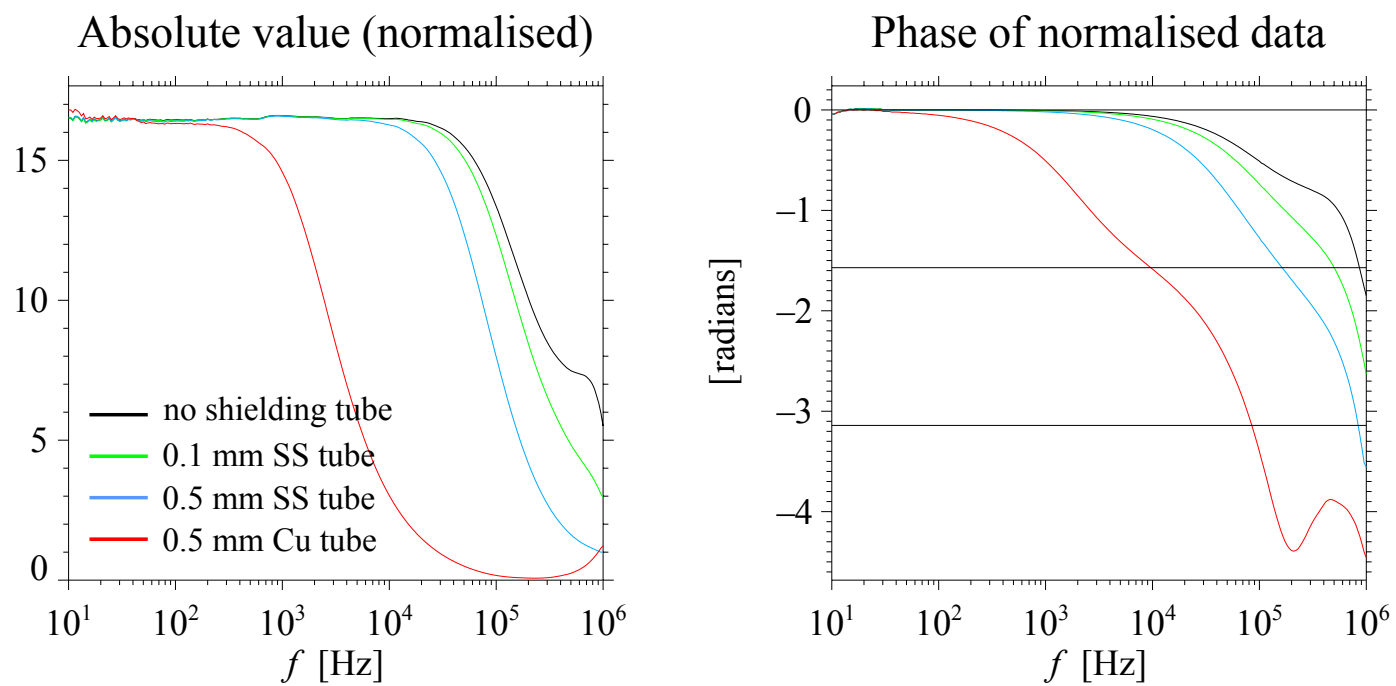

Figure 20: Absolute value (left) and phase (right) of the response of a single $R C$ solenoid in the MPTS. The response is normalised to the response of a rectangular flux loop with a single winding and very low self-inductance. The response is shown for the naked solenoid (black) and the same solenoid inserted in SS tubes with $0.1 \mathrm{~mm}$ and $0.5 \mathrm{~mm}$ wall thickness and in a $\mathrm{Cu}$ tube with $0.5 \mathrm{~mm}$ wall thickness.

The deviations of the "naked" solenoid above $10 \mathrm{kHz}$ from the "ideal" response are due to its self-inductance. If signals up to and above $1 \mathrm{kHz}$ should be measured, both SS shielding tubes are still acceptable, but the $0.5 \mathrm{~mm} \mathrm{Cu}$ tube is not, due to the large eddy currents induced in it. Based on the results of these measurements and on the bending behaviour, SS tubes with $0.25 \mathrm{~mm}$ wall thickness were chosen as microwave stray radiation shielding for the in-vessel RCs.

\subsubsection{Dimensioning and thermal balance of final design for in-vessel Rogowski coils}

With a conservative assumption of $3 \%$ microwave absorption at the surface of the SS shielding tubes and $50 \mathrm{~kW} / \mathrm{m}^{2}$ stray radiation level, we obtain a thermal load of $40 \mathrm{~W} / \mathrm{m}$ for each shielding tube. Under the same assumptions as for the DLs, a maximum of $\sim 30 \mathrm{~W} / \mathrm{m}$ additional thermal load from the back faces of WPEs must be taken into account. The length of the different in-vessel RC segments varies between $200 \mathrm{~mm}$ and $1000 \mathrm{~mm}$. Depending on their length, they have 0-4 intermediate holders between their 
end holders, such that a maximum of $\sim 200 \mathrm{~mm}$ length between neighbouring attachments to the PV is kept. However, the thermal conductance of the thin SS shielding tubes is so low that the heat flux of $7 \mathrm{~W}$ to be transmitted along a tube close to an end or intermediate holder would require a temperature gradient of $\sim 70 \mathrm{~K} / \mathrm{mm}$. If the conduction along the core tube and along the winding structure is taken into account, the required temperature gradient would still amount to $\sim 25 \mathrm{~K} / \mathrm{mm}$.

Therefore, $\mathrm{Cu}$ heat counduction rails are inserted between the shielding tubes with a cross-sectional area of $37 \mathrm{~mm}^{2}$. The cross-section of these rails is designed such as to provide a good contact with the shielding tubes (see fig. 21), in particular when the entire

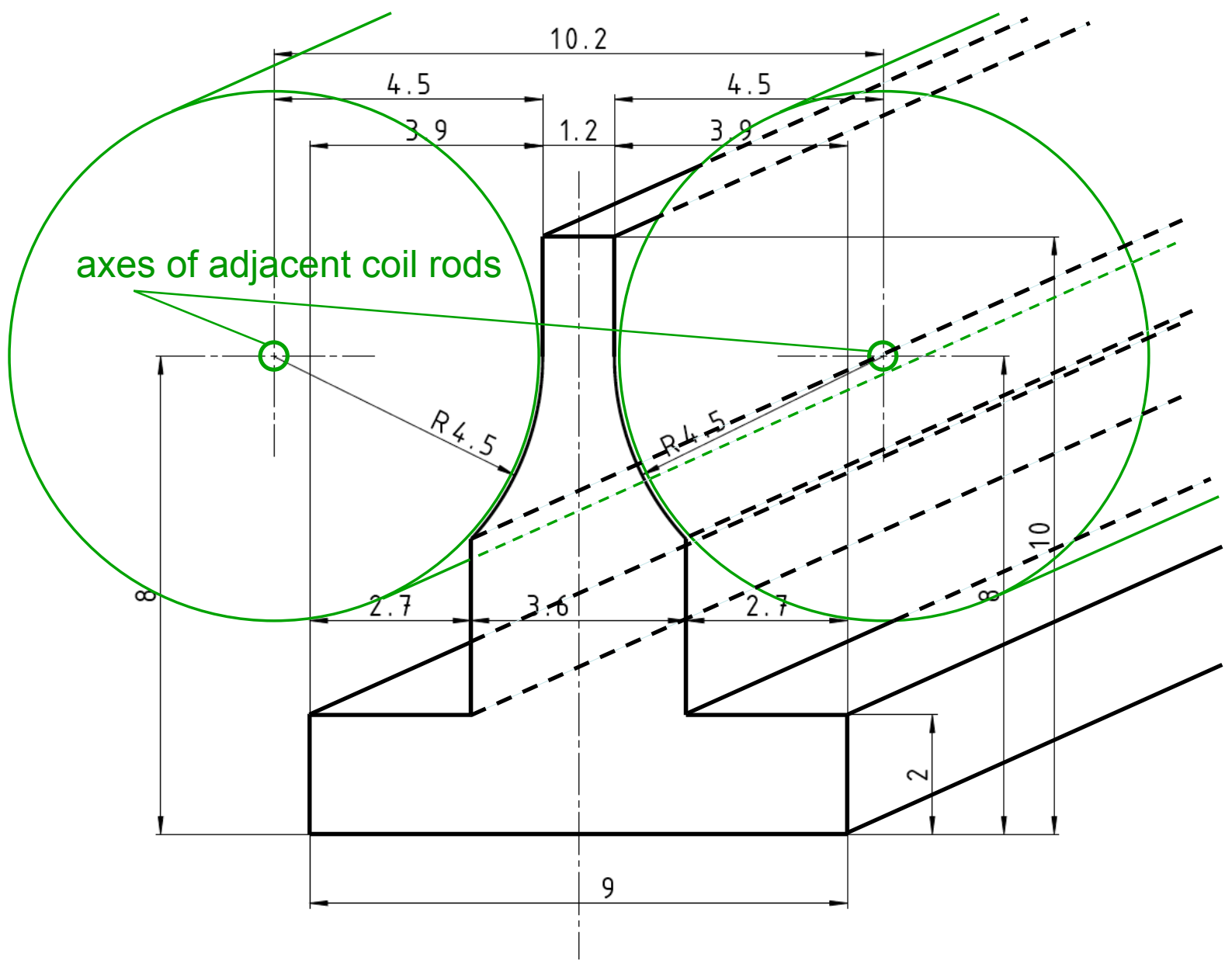

Figure 21: Cross section of a heat conduction rail for the in-vessel RCs (dimensions in $\mathrm{mm})$.

package is pressed together by the end and intermediate holders and additional steel straps. The upper part of the rails is intersected every $20-40 \mathrm{~mm}$ such that they can be adapted to the curved shape of the solenoids during the assembly of the segments (see fig. 19); hence, only the lower $9 \mathrm{~mm} \times 2 \mathrm{~mm}$ are continuous.

Each of the two outermost $\mathrm{Cu}$ rails of a segment has to carry the heat deposited on the outermost shielding tube, plus a fraction of the heat deposited on the next shielding tube. For our estimate of temperatures we consider therefore one of the outermost solenoids, and we assume an effective cross-sectional area of only $30 \mathrm{~mm}^{2}$ for the $\mathrm{Cu}$ rails to account 
for the intersections in the upper part of their profile. The maximum temperature difference along the parabolic temperature profile (which arises between two neighbouring end or intermediate holders if only heat conduction is considered) will then amount to $\sim 40 \mathrm{~K}$. We estimate additional $\sim 60 \mathrm{~K}$ from the stagnation point along the circumference of the outermost shielding tube to the contact area with the $\mathrm{Cu}$ rail and $\sim 40 \mathrm{~K}$ to account for only localised contact between shielding tube and $\mathrm{Cu}$ rail (see below). The $\mathrm{Cu}$ rails are pressed to the $\mathrm{Cu}$ adapter blocks by the end and intermediate holders. We insert graphite layers of $0.2 \mathrm{~mm}$ thickness to improve the reliability of the thermal contact. A graphite layer of $1 \mathrm{~mm}$ thickness is inserted between the adapter block and the plasma vessel (see section 3.4). For both interlayers, we assume heat transmission coefficients of $2000 \mathrm{~W} /(\mathrm{Km})$, although our tests showed higher heat transmission coefficients for the thinner layers. The two interfaces will add $\sim 40 \mathrm{~K}$ (between outermost $\mathrm{Cu}$ rail and adapter block) and $\sim 20 \mathrm{~K}$ (between adapter block and plasma vessel) to our temperature chain. Adding up these temperature steps to the maximum plasma vessel background temperature of $60^{\circ} \mathrm{C}$, we estimate a maximum temperature of $\sim 260^{\circ} \mathrm{C}$ at the outermost shielding tubes. However, the winding wire is separated from the shielding tube by a layer of glass fibre mesh and it has a better heat conduction around the solenoid than the SS shielding tube. We therefore estimate that its temperature will not exceed the limit of $250^{\circ} \mathrm{C}$ locally.

To verify these estimates, an ANSYS finite element thermal model was used to calculate the maximum temperature to be expected in the $\mathrm{RC}$ windings. In particular, we wanted to test the influence of different assumptions about the thermal contact between the $\mathrm{Cu}$ heat conduction rails and the shielding tubes. To this end, the surface of the $\mathrm{Cu}$ rails was subdivided into sub-surfaces of $\sim 20 \mathrm{~mm} \times 3 \mathrm{~mm}$ (see fig. 22). We then compared

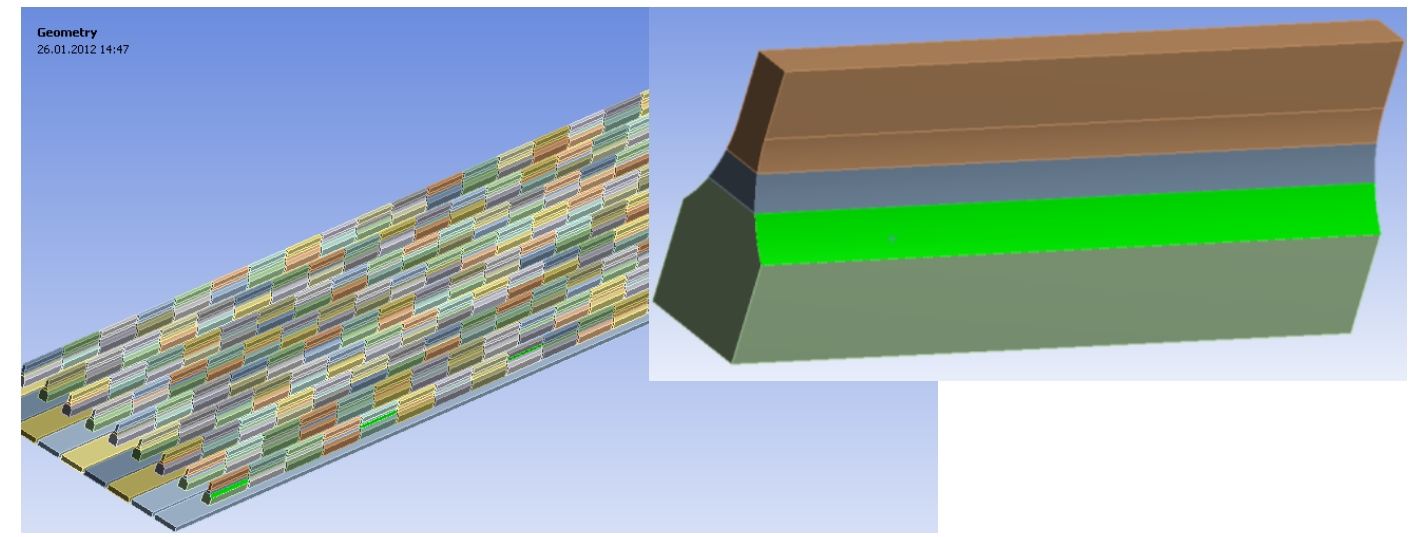

Figure 22: Subdivision of the surfaces of the $\mathrm{Cu}$ heat conduction rails between the individual solenoids of an $R C$ segment. The potential contact area between the rails and the shielding tubes of the solenoids is subdivided into sub-surfaces of $\sim 20 \mathrm{~mm} \times 3 \mathrm{~mm}$, such that a fraction of these sub-surfaces can be chosen by random to simulate the poorly defined thermal contact between heat conduction rails and shielding tubes.

the cases

(1) full thermal contact between $\mathrm{Cu}$ rails and shielding tubes, 
(2) thermal contact at randomly chosen 6 out of the 57 sub-surfaces between each railtube pair (10\% contact case), and

(3) only radiative heat transfer between $\mathrm{Cu}$ rails and shielding tubes.

In the stationary case, the resulting maximum temperatures within the winding were (1) $140^{\circ} \mathrm{C}$, (2) $260^{\circ} \mathrm{C}$, (3) $300^{\circ} \mathrm{C}$, if all graphite tiles on top of the segment were loaded with $500 \mathrm{~kW} / \mathrm{m}^{2}$. We consider the $10 \%$ contact case the most realistic of the three cases. If, still more realistically, only one row of tiles is highly loaded $(0.1 \mathrm{~m}$ in poloidal direction, compare section 3.3), the maximum winding temperature is reduced to $220^{\circ} \mathrm{C}$ in the $10 \%$ contact case. As an example of the analysis results, we show the temperature distribution on the surface of the shielding tubes for this simulation case in fig. 23. The blotchy pattern

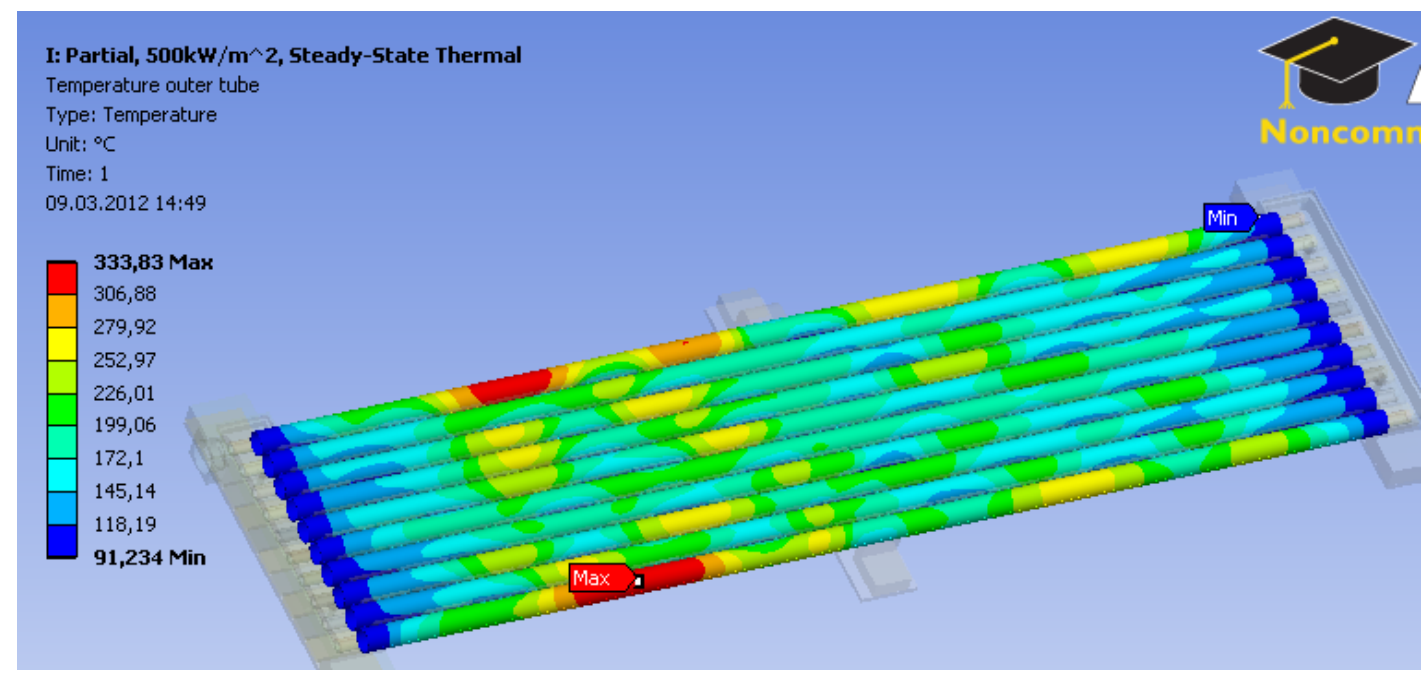

Figure 23: Temperature distribution on the shielding tubes resulting from one of the simulation cases, as described in the text. In this case, a perfect thermal contact between heat conduction rails and shielding tubes is realised in $10 \%$ of the sub-surfaces shown in fig. 22. The resulting large temperature differences reflect the poor thermal conduction along the SS shielding tubes.

reflects the randomly chosen contact areas between the $\mathrm{Cu}$ heat conduction rails and the shielding tubes.

These results are valid, if the direct thermal radiation from the back faces of the graphite tiles is shielded by additional $\mathrm{Cu}$ sheets thermally connected to the heat sinks of the WPEs. Without these thermal shields, the maximum temperature of the winding for the case with $10 \%$ contact area and $500 \mathrm{~kW} / \mathrm{m}^{2}$ load on all tiles increases from $260^{\circ} \mathrm{C}$ (see above) to $350^{\circ} \mathrm{C}$, which is entirely unacceptable.

Like in the DLs, Pt1000 temperature sensors are installed for temperature surveillance inside the core tubes of the outermost solenoids of several RC segments in locations where particularly high thermal loads from the back faces of WPEs are expected.

A conceptual design mock-up of an in-vessel RC segment was tested in MISTRAL [33] to demonstrate the ECR radiation shielding of the design: Three Rogowski solenoids of $300 \mathrm{~mm}$ length with SS shielding tubes of $8.8 \mathrm{~mm}$ outer diametre and $0.25 \mathrm{~mm}$ wall thickness and $0.7 \mathrm{~mm}$ holes for venting were inserted in closed SS end holders. These were 
screwed to stud bolts welded onto a water-cooled SS ground plate representing the PV. A $\mathrm{Cu}$ adapter block was placed between each end holder and the ground plate with graphite foil layers of $0.2 \mathrm{~mm}$ thickness above and below the $\mathrm{Cu}$ adapter block. Between every two shielding tubes, a $\mathrm{Cu}$ sheet of $12.5 \mathrm{~mm}^{2}$ cross-sectional area was clamped as a substitute for the milled heat conduction rails of the final design. Thermocouples were placed in the centres of the core tubes of each of the three solenoids. A further thermocouple was placed into one end holder. In addition, the resistance of the middle solenoid winding was measured in four-wire technique to derive the average temperature of the winding.

MISTRAL was operated with this mock-up for up to 30 min with a stray radiation power density of $\sim 50 \mathrm{~kW} / \mathrm{m}^{2}$. In fig. 24 , the temperature evolution measured with ther-

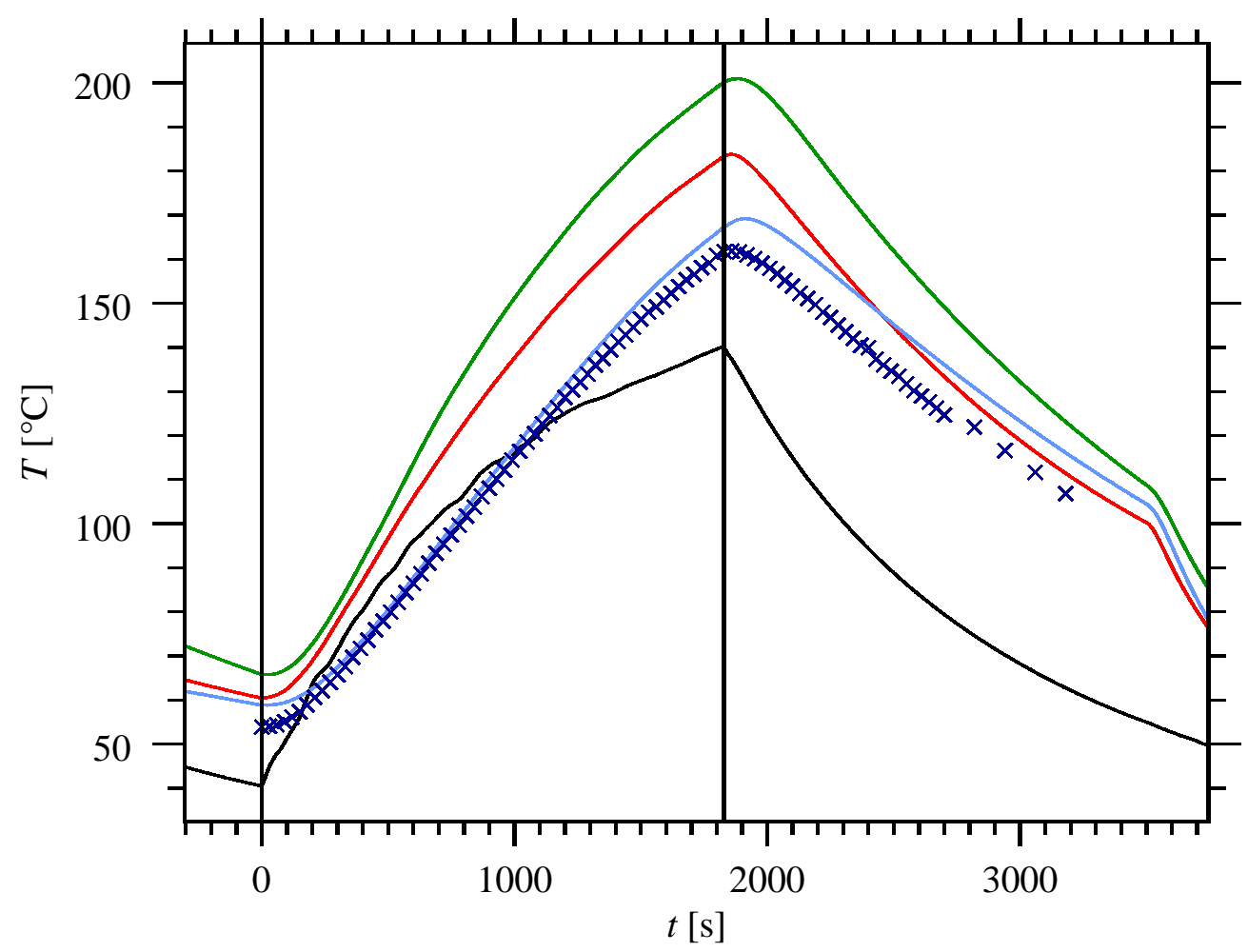

Figure 24: Test of an in-vessel RC mock-up in MISTRAL with a stray radiation power density of $\sim 50 \mathrm{~kW} / \mathrm{m}^{2}$. The microwave power was applied in the $30 \mathrm{~min}$ interval between the two vertical lines. Temperatures were measured by thermocouples in the middle of the core tubes of the three solenoids (light blue: middle solenoid; red and green: outer solenoids) and in one of the end holders (black). The average winding temperature of the middle solenoid was also calculated from the ohmic resistance (dark blue crosses).

mocouples in one of the end holders and in the centres of the three solenoid core tubes is shown together with the average temperature of the middle solenoid winding as derived from its ohmic resistance. From the time delay of 30-60 s relative to the switching of the microwave power we can see that it takes some time before the heat is transmitted to the core tubes and to the thermocouples inserted. The average winding temperature reacts faster to the switching. Depending on the temperature profile along the solenoid, which would be parabolic if heat conduction along the segment dominates, we must assume that the peak temperature in the winding was higher than measured in the core tube. The 
outer solenoids reached higher core temperatures than the middle one. This is expected since the middle solenoid is in contact with two $\mathrm{Cu}$ sheets for heat conduction to the end holders, while each of the outer solenoids is in contact with only one of the $\mathrm{Cu}$ sheets, and the middle solenoid is shielded better by the two adjacent $\mathrm{Cu}$ sheets.

We note that, after $30 \mathrm{~min}$, the arrangement is not yet stationary, and that the equilibrium maximum temperature of the windings would therefore most probably exceed the value of $250^{\circ} \mathrm{C}$ specified as operational limit of the polyimide-insulated winding wire. The thermocouple in the end holder reacts very quickly to the switching of the microwave power. This indicates that a significant amount of microwave power could penetrate into the end holder. The final design of the heat conduction rails and of the end holders was improved accordingly, as detailed above.

After the stray radiation test, the wires in the end holders did not show any sign of damage, and the middle solenoid passed the electrical tests (ohmic resistance of the winding unchanged, $2 \mathrm{kV}$ for 1 min between winding and core and shielding tubes).

Apart from the information on the temperature evolution under thermal load, due to the microwave power absorbed in the metallic housing, the MISTRAL test demonstrated that the shielding is sufficient to prevent a microwave radiation level which could damage the insulation of the winding wire and of the signal cables.

\subsubsection{Electromagnetic forces}

Due to the high electric conductance of the $\mathrm{Cu}$ heat conduction rails, the electromagnetic forces due to poloidal eddy currents must be considered. The most critical load case is a fast shutdown of the W7-X superconducting magnet system, inducing a poloidal loop voltage of up to $0.4 \mathrm{~V} / \mathrm{m}$. This could drive a current of almost $900 \mathrm{~A}$ in a single heat conduction rail, pulling the rail toward the plasma vessel. The rails are clamped between the shielding tubes and supported by the adapter blocks below the end and intermediate holders. Only the friction due to the clamping force and the restoring force will prevent the sections between the holders from being extracted. The cross-section of the final design does not allow the rails to be pushed toward the plasma centre, such that eddy currents due to a fast decay of the plasma energy are less critical.

In an in-vessel RC prototype, the force necessary to extract a single heat conduction rail between the adjacent shielding tubes was measured. According to this test, and allowing for a safety factor of 2 , the line force on a heat conduction rail should not exceed $375 \mathrm{~N} / \mathrm{m}$. In the maximum magnetic field of $4 \mathrm{~T}$, which can occur in some locations at the W7-X PV wall, this corresponds to a current of $\sim 90 \mathrm{~A}$, which is only $10 \%$ of the current which could be induced in the critical load case.

Three measures (summarised in fig. 25) are taken to increase the ohmic resistance on the current path from the plasma vessel to the $\mathrm{Cu}$ rails, along the rails and back to the plasma vessel:

1. At the end holders, graphite layers of $0.2 \mathrm{~mm}$ thickness are inserted not only between the $\mathrm{Cu}$ rails and the adapter block but also above the $\mathrm{Cu}$ rails, between end holder and $\mathrm{Cu}$ rails. This way, the interface resistance between plasma vessel and heat conduction rail can be reliably increased to $\sim 4 \mathrm{~m} \Omega$ per rail at each end holder (see 


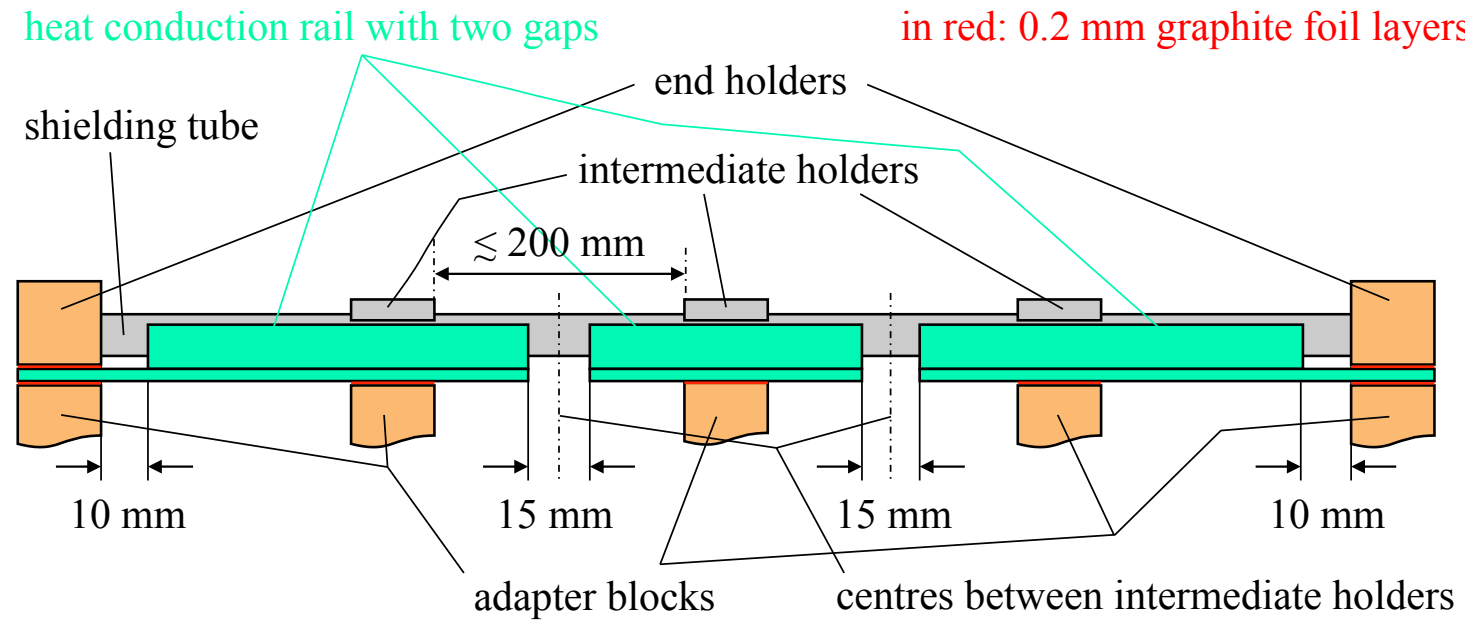

Figure 25: Section along an in-vessel $R C$ segment, illustrating the $15 \mathrm{~mm}$ gaps in the heat conduction rails between any two intermediate holders, the $10 \mathrm{~mm}$ gaps between the upper part of the heat conduction rail profile and the end holders, and the positions of graphite layers of $0.2 \mathrm{~mm}$ thickness.

section 3.4).

2. In order to avoid a short circuit between end holder and rail through the shielding tubes, the upper part of the rail profile is removed on a length of $10 \mathrm{~mm}$.

3. In segments with more than two intermediate holders, gaps of $15 \mathrm{~mm}$ were left in the $\mathrm{Cu}$ rails in the middle between intermediate holders, at the stagnation points of the temperature profile along the segment, where the heat flux along the segment is close to 0 . We estimate that these gaps will further increase the maximum temperature of the shielding tubes by $15 \mathrm{~K}$, which is still tolerable.

Simulations of the currents in the resulting network of resistances formed by the sections of heat conduction rails and shielding tubes and by the different interfaces show that the maximum current of $90 \mathrm{~A}$ is no longer exceeded in any section of heat conduction rail in this design.

\subsection{Expected signals}

In an ideal RC consisting of solenoids in the dimensions used in W7-X, with 5 solenoids per segment connected in series, a magnetic flux of $0.62 \ldots 0.63 \mu \mathrm{Vs}$ is generated per Ampère penetrating the surface spanned by the CRC path. This corresponds to $125 \mathrm{mVs}$ for the maximum net toroidal plasma current of $200 \mathrm{kA}$, to be expected in certain current drive scenarios. A measurement accuracy of $100 \mathrm{~A}$ would correspond to a magnetic flux of $\sim 60 \mu \mathrm{Vs}$ penetrating the $\mathrm{CRC}$ circuit.

In a single (half) segment, the flux generated by a current filament is reduced by the ratio of the arc length the segment spans, as seen from the current filament, to the arc length $2 \pi$ of a full circle. In the TP, this corresponds roughly to the length of the segment 
relative to the full poloidal circumference of the PV. This fraction can be as low as $4-5 \%$ for the shortest segments.

The largest signal amplitude to be expected in a CRC will occur if, starting from a discharge with a net toroidal plasma current of $200 \mathrm{kA}$, the plasma decays with an energy confinement time of $10 \mathrm{~ms}$. The resulting voltage in an in-vessel $\mathrm{CRC}$ circuit would be $12.5 \mathrm{~V}$. In the CRCs outside the plasma vessel, the voltage would be reduced to $5 \mathrm{~V}$, due to the longer time scale for toroidal currents in the plasma vessel of $\sim 25 \mathrm{~ms}$ (see section 2.2). The largest signal amplitude induced in single (half) segments would be smaller, corresponding to their arc length, as detailed above.

For an absolute accuracy of $100 \mathrm{~A}$ in a half-hour discharge, the signal amplitude of a CRC circuit must be measured with an accuracy of $35 \mathrm{nV}$.

For the CRCs and SRCs outside the PV, the time resolution is restricted to $\sim 25 \mathrm{~ms}$ by the time scale for toroidal currents in the PV. For the in-vessel RCs, it was shown in section 6.1.2 that the ECR radiation shielding tube only slightly reduces the time resolution of a single solenoid. However, for the series connection of several of these solenoids or even of several segments, the larger self-inductance of such a circuit will (even without shielding tubes) reduce the time resolution.

In an ideal CRC, changes of currents which do not penetrate the opening of the CRC do not induce a net voltage. However, inhomogeneities in the winding area density and, in particular, gaps in the winding destroy this ideal property. Changes in the field coil currents can therefore induce signals in our non-ideal CRCs, and the field coil currents must therefore be recorded. The upper limit for the net magnetic flux through a W7-X in-vessel CRC circuit, due to the gaps between segments, is $\sim 0.2 \mathrm{Vs}$ at a field coil current of $18 \mathrm{kA}$. A measurement accuracy for the net toroidal plasma current of $100 \mathrm{~A}$ would therefore correspond to a measurement accuracy of $5 \mathrm{~A}$ in the field coil currents. Such an accuracy is provided by the power supplies of the W7-X superconducting coils.

A further perturbation of the measurements by CRCs and SRCs is due to thermal expansion, of the cross section of the solenoids as well as of the length of the solenoids. The overall uncertainty due to this effect in the measurement of the net toroidal plasma current may be up to $17 \mathrm{kA}$ for a temperature change of $200 \mathrm{~K}$ of the in-vessel RCs, depending on the true coupling between the Rogowski and the field coil circuits. This effect will be much smaller for the RCs outside the plasma vessel, which may help to distinguish it from signals due to changes in the plasma current.

\section{Mirnov coils}

\subsection{Design and testing}

\subsubsection{Basic design}

For the Mirnov coils, we had the goal to avoid altogether a metallic housing for ECR stray radiation shielding, since it damps the high-frequency response and shifts its phase. For the same reason, the Mirnov coils should have low self-inductance, which is supported by a low winding density. Although for the frequency range up to $1 \mathrm{MHz}$ (see section 2.1.5) a housing with a thin metallic film could be a possible solution, we decided to make 
use of the low winding density and chose a design suitable for a winding wire without insulation, by guiding it in slots of the coil former (see fig. 26). The coil former should

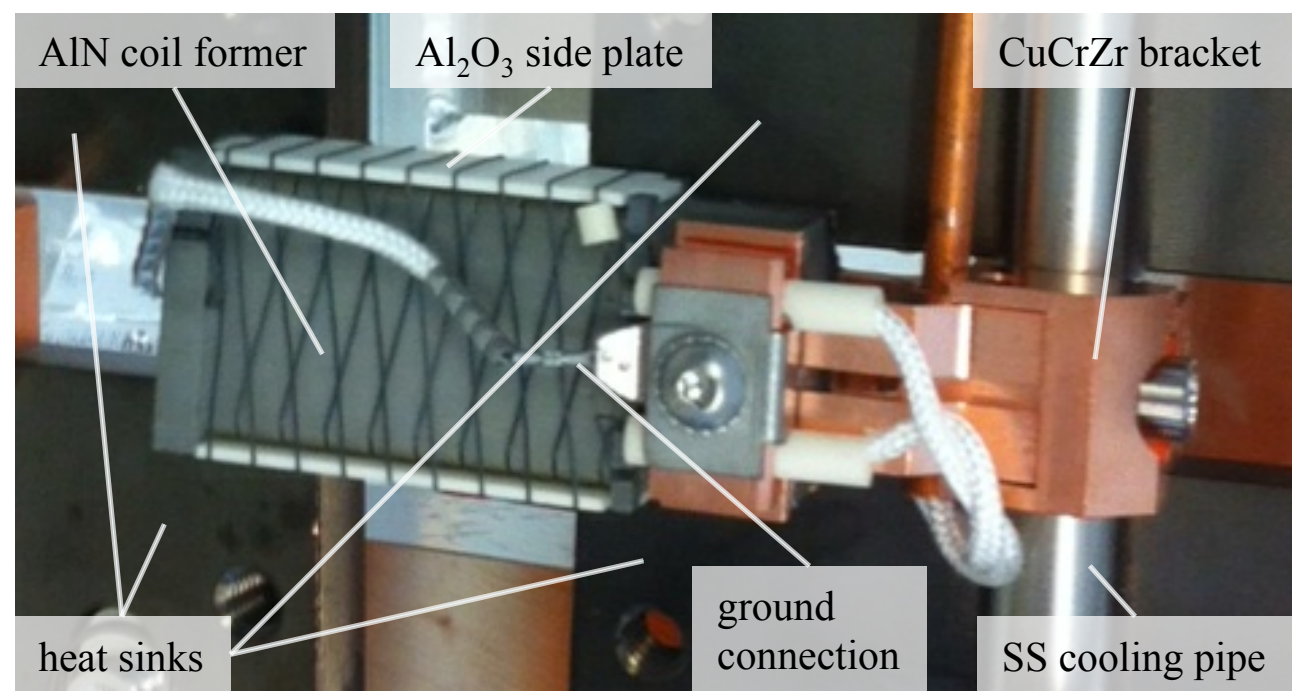

Figure 26: Mirnov coil behind the heat sinks of a wall protection element. The coil is attached to a SS cooling pipe by a $\mathrm{CuCrZr}$ clamp (right). The Constantan ${ }^{\circledR}$ winding wires of the two winding layers continue through a $\mathrm{Cu}$ pipe (toward the top right). Inside the $\mathrm{Cu}$ pipe, the two wires are twisted and insulated by silicate sleevings. The other ends of the winding wires are crimped together and connected to ground, such that the winding has a middle connection to ground.

be made of ceramics, which can support high temperatures. Nevertheless, since ceramics typically have a significantly larger microwave absorption than metallic surfaces, a type of ceramics with high heat conductivity is required, in order to limit the temperatures by attaching the coils to water-cooled components.

For the desired spatial distribution of Mirnov coils in the plasma vessel, some of them have to be placed in regions covered by graphite tiles and some in regions covered by SS panels as wall protection elements. Therefore, two different types of coil were designed. Due to the decay of the amplitude of magnetic perturbations $\propto r^{-(m+1)}$ (see section 2.1.5), the coils should also be located as close as possible to the plasma axis.

7.1.1.1 Coils behind gaps between wall protection tiles In the regions of the PV protected by graphite tiles, the Mirnov coils are located behind the gaps between neighbouring tiles. The tiles can thus still protect the coils from the full heat load of the plasma. The coils are arranged with their axis parallel to the gap, such that no well-conducting current path in parallel with the winding exists.

Two layers of wire are wound on the ceramic coil former with 9 windings each. Between the two layers, there is a ground connection in order to prevent potential DC charging of the coil circuit due to plasma contact of the open winding wire. The self-inductance of the entire coil is $\sim 3.7 \mu \mathrm{H}$. The winding wire of the lower layer is guided by slots in the edges of the coil former. The upper layer is separated from the lower layer by additional $\mathrm{Al}_{2} \mathrm{O}_{3}$ plates, again with slots along their edges (see fig. 26). The winding wires are also forming the first section of the signal cables: To this end, they are not cut directly at the 
coil, but a length of $\sim 2 \mathrm{~m}$ is left. Each of the two wires is threaded through a silicate sleeve (see fig. 26), the two wires are then twisted and threaded through a $\mathrm{Cu}$ pipe with $4 \mathrm{~mm}$ outer diametre and $1 \mathrm{~mm}$ wall thickness. This pipe is attached along the cooling pipes of the WPE and finally enters into a connection box machined from $\mathrm{Cu}$, attached to the PV, where crimp connections to the standard type of in-vessel signal cables (see section 8) are created.

Each coil is at one end screwed to a CuCrZr bracket, which is contacting the coil former at two faces (visible in fig. 27) with $0.2 \mathrm{~mm}$ graphite interlayers between the coil

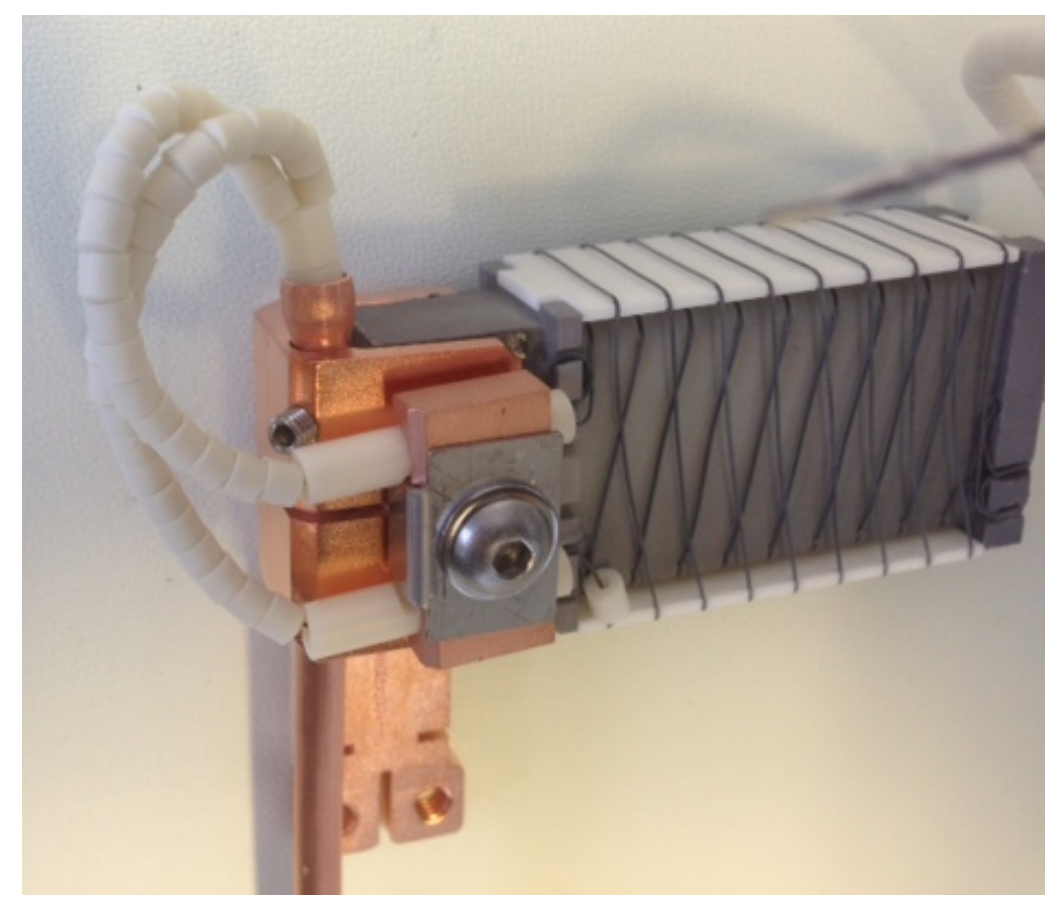

Figure 27: Mirnov coil with lateral holder. On the left hand side of the coil former, contact surfaces with $0.2 \mathrm{~mm}$ graphite interlayers between holder and coil former exist on two sides of the coil former for good heat transmission.

former and the bracket. The brackets are clamped to the cooling pipes of the heat sinks, again with $0.2 \mathrm{~mm}$ graphite interlayers to provide good thermal contact even in case of a slight mismatch of the contact surfaces.

The Mirnov coils were attached to the WPEs, and their positions and orientations were surveyed geometrically relative to the corresponding WPE before its integration into the PV. The survey results will be used together with later surveys of the WPEs and of the $\mathrm{PV}$ to determine the coil positions and orientations after the integration of the WPEs into W7-X.

7.1.1.2 Coils on the plasma side of wall protection panels In those regions of the PV where lower thermal loads are expected, the PV surface is protected by watercooled SS panels. Roughly, this is the case on the torus outboard side except around the TPs. The Mirnov coils in these regions are those forming the outboard parts of the (incomplete) poloidal arrays close to the BPs, and those forming the outboard part of the toroidal array (see fig. 1). 
All Mirnov coils on the wall protection panels will not be installed before the first operation phase of W7-X, and no detailed design does exist so far. The basic idea is to protect these coils from the plasma by graphite caps. For long-pulse discharges, it is important that a good thermal contact between the graphite caps and the water-cooled panels is maintained. However, the panels may deform by several millimetres under the pressure of the cooling water [28] as well as due to the thermal load [41]. Even though the graphite caps for Mirnov coils will be much smaller than an entire panel, springs and/or an articulated design of the graphite caps will be required to maintain the contact pressure between the caps and the panels, which is necessary for good heat conduction. When designing the graphite caps, a trade-off between sufficient heat conductance (thicker walls of graphite cap) and sufficiently low electric conductance (thinner walls) must be found to keep eddy currents in the direction of the winding sufficiently small. Possibly, the graphite cap must be designed with a gap filled by an $\mathrm{AlN}$ or $\mathrm{Al}_{2} \mathrm{O}_{3}$ plate.

The signal cables could be realised in the same design as for the Mirnov coils behind the graphite tiles, with the $\mathrm{Cu}$ tube running behind graphite caps on the plasma side of the panels, and then passing through the gaps between panels to connection boxes installed on the PV wall.

\subsubsection{Time resolution}

To verify the response of the Mirnov coils, a prototype coil was tested in the frequency range of interest in the MPTS (see section 3.2). The results are shown in fig. 28. The inductance of the coil and the capacitance of the signal cable form a resonant circuit, which can result in higher than expected signal voltages. Therefore, in addition to the coil, a circuit of coil and $6 \mathrm{~m}$ of the shielded twisted-pair signal cable used in the PV was tested. The difference in the signal amplitude becomes notable above $\sim 300 \mathrm{kHz}$, whereas the difference in phase remains moderate. This demonstrates that the resonance frequency of this circuit is above the frequency range up to $1 \mathrm{MHz}$ which is expected for magnetic modes in W7-X.

For each of the 125 series Mirnov coils mounted behind the gaps between wall protection tiles, the signals induced in the coil by magnetic fields of $100 \mathrm{~Hz}-2 \mathrm{MHz}$ parallel to the coil axis and in the two directions perpendicular to the coil axis were measured. This served as test and as documentation, how sensitive the individual coils are to oscillations in magnetic field components not parallel to the coil axis. The latter information is of interest because the winding area perpendicular to the coil axis is rather small, and there are sections of the winding wire enclosing considerable areas perpendicular to the other two directions in space (visible in figs. 26 and 27). In fig. 29 we show the average values and standard deviations of these measurements. The signals from an oscillating field along the coil axis are normalised to the signals of the one-turn calibration loop. Their deviation from an ideal response is small, and the variation between different Mirnov coils, represented by the standard deviations of amplitude and phase, calculated separately for each frequency, is also small.

The signals due to magnetic field oscillations in the two directions perpendicular to the coil axis are normalised to the signals along the coil axis individually for each coil. Their amplitude is, on the average, $2-5 \%$ of the signal amplitude for magnetic field oscillations 


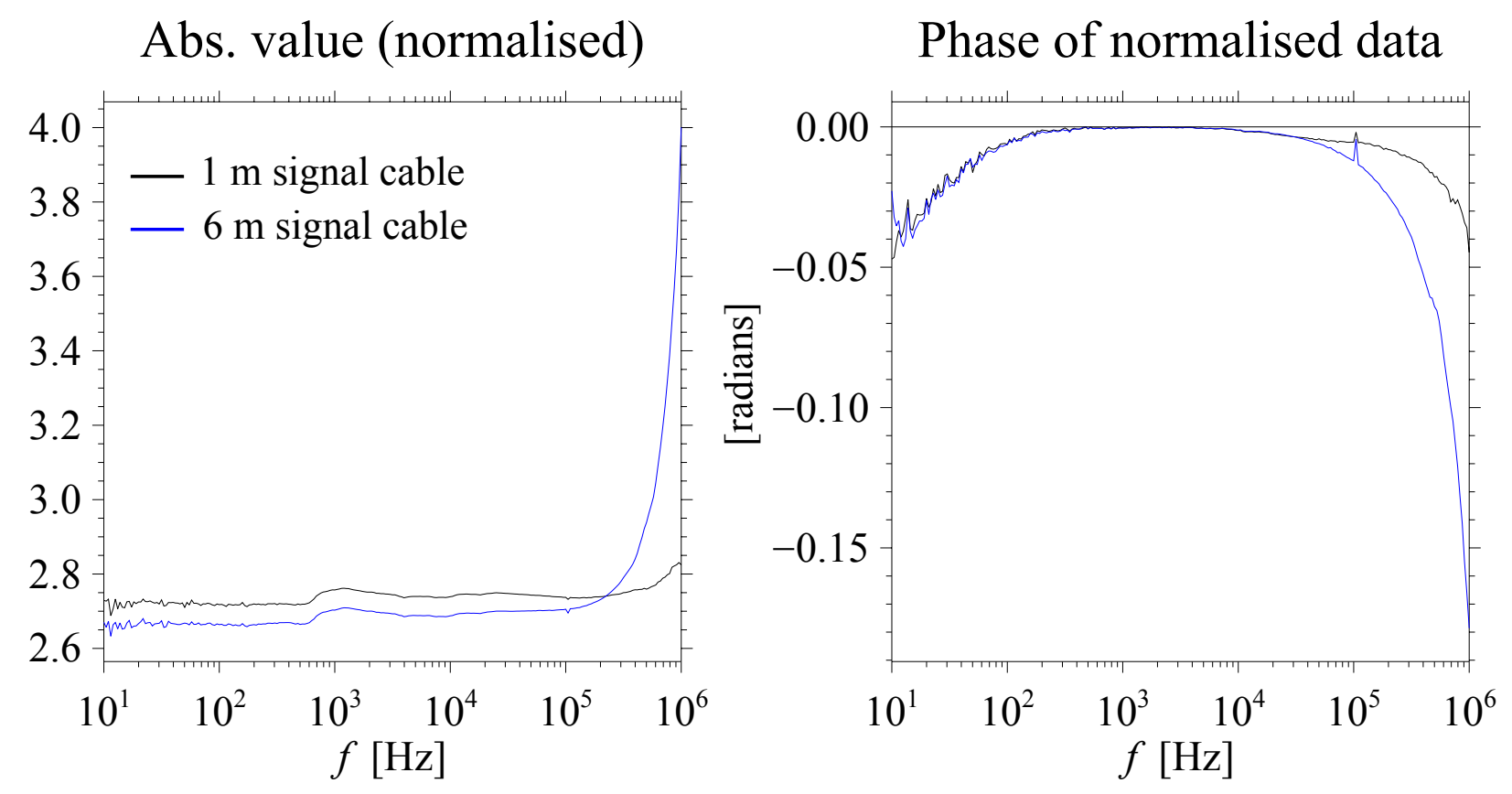

Figure 28: Amplitude and phase of the response in the MPTS of a prototype of the Mirnov coils to be installed behind gaps between wall protection elements. The black and blue curves are for different lengths of signal cable between the Mirnov coil and the pre-amplifier. The cables were of the same type as the polyimide-insulated shielded twisted-pair cables used as signal cables in the PV. The deviations between the two cases at high frequencies are due to the cable capacitance. All signals are normalised by those of a simple one-turn quadratic flux loop of $2646 \mathrm{~mm}^{2}$ winding area with $1 \mathrm{~m}$ of signal cable. The induced voltages below $100 \mathrm{~Hz}$ are very low. This could be the reason for the increased noise and the slight deviation of the phase from 0.

along the coil axis. Since the variation between the individual coils for these directions is large, the standard deviations for amplitudes above and below the average were calculated separately. The phase of these signals relative to the phase of signals from magnetic field oscillations along the coil axis mostly are 0 or $\pi$, depending on the orientation of the winding area in the directions perpendicular to the coil axis. Intermediate phase values also occur, indicating capacitive signal components. A representation of average phase values of the signals for these magnetic field directions would therefore not be very useful.

To summarise, for magnetic fields along the Mirnov coil axis (this will be approximately the poloidal direction in $\mathrm{W} 7-\mathrm{X}$ ), all series coils exhibit a rather uniform and ideal behaviour in the frequency range $100 \mathrm{~Hz}-2 \mathrm{MHz}$. In comparison with magnetic field components along the coil axis, the influence of other magnetic field components is small.

\subsubsection{Thermal balance and choice of materials}

Different ceramics were considered suitable for the coil formers of the Mirnov coils. We therefore tested prototype coils made of aluminum nitride (AlN), boron nitride (BN), aluminum oxide $\left(\mathrm{Al}_{2} \mathrm{O}_{3}\right)$, and $\mathrm{BN}+\mathrm{ZrO}_{2}$ in MISTRAL at microwave powers of $\sim 35 \mathrm{~kW} / \mathrm{m}^{2}$. The prototypes were attached to a water-cooled SS base plate with a graphite foil interlayer or with $\mathrm{Cu}$ brackets. The temperature of each coil former was measured by 


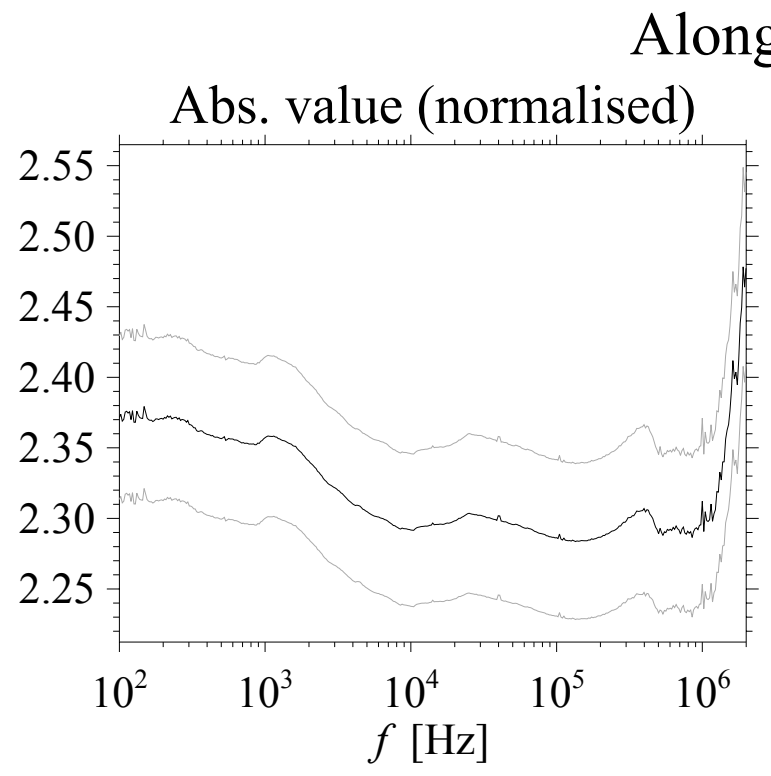

In toroidal direction: Abs. value (normalised)

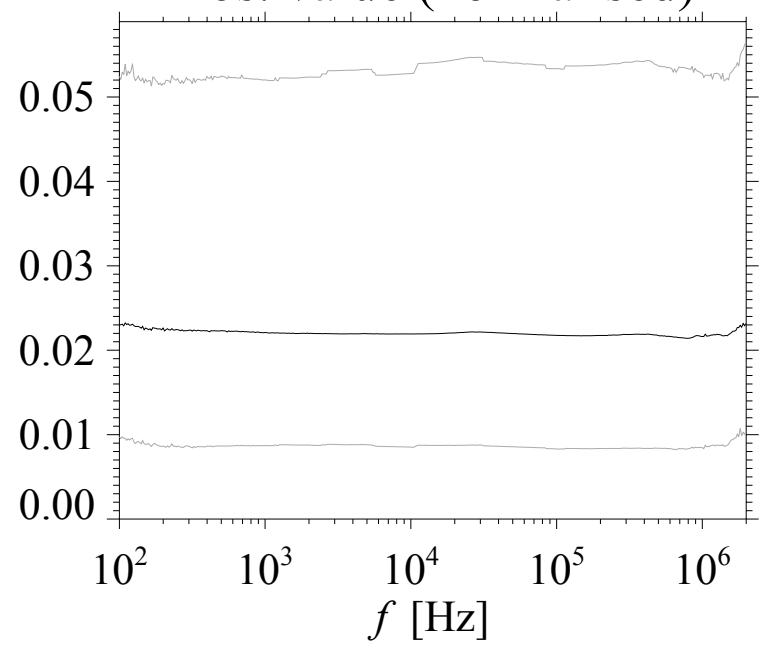

Phase of normalised data [radians]

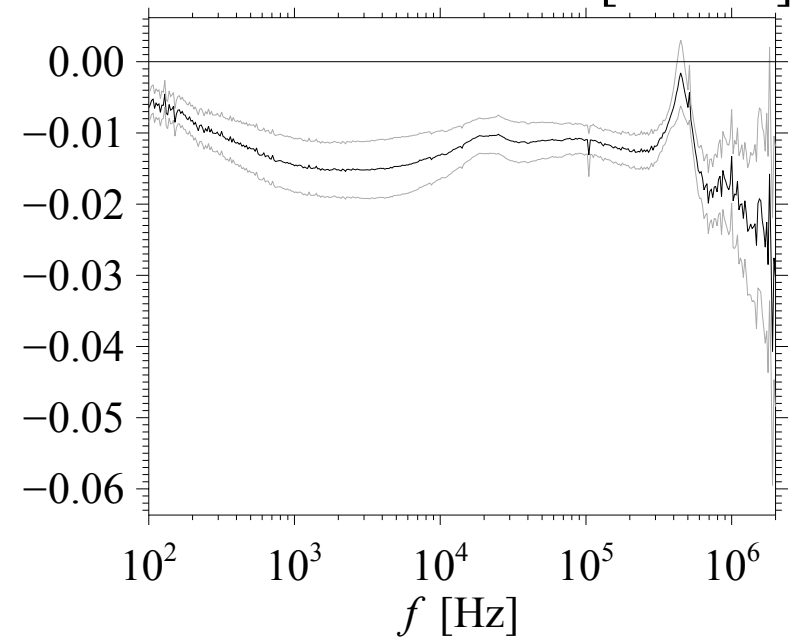

In radial direction: Abs. value (normalised)

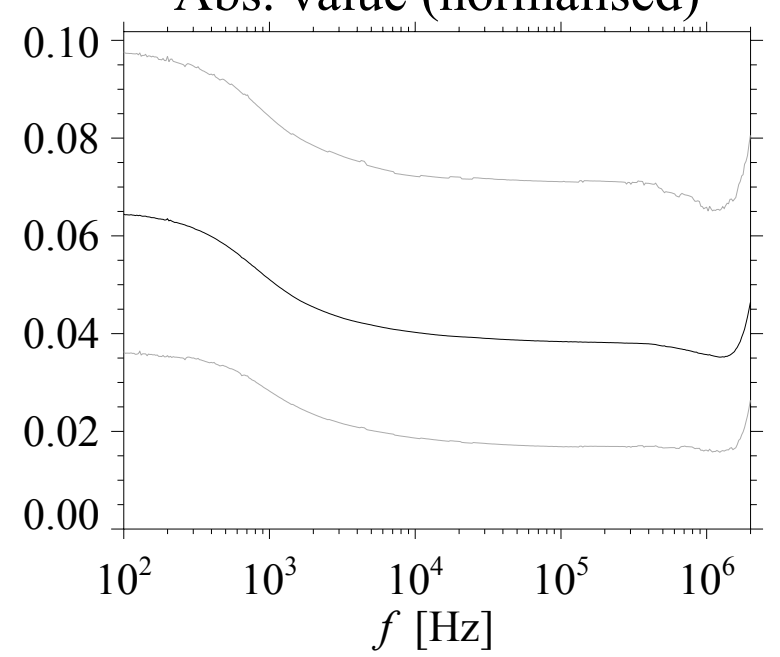

Figure 29: Amplitudes and phases of the response in the MPTS of all 125 Mirnov coils installed behind gaps between wall protection elements. The response of each coil to excitation in all three directions relative to the coil axis was measured. The signals from the oscillating magnetic field along the Mirnov coil axis are normalised to the signals measured by a simple one-turn quadratic flux loop of $2646 \mathrm{~mm}^{2}$ winding area, the signals from the oscillating field along the other two axes are normalised to the signals with the magnetic field along the coil axis of the same coil. The black curves are the average values, the grey curves denote the standard deviations. For the magnetic field direction perpendicular to the coil axis, the standard deviations were calculated separately for those amplitudes above and below the average.

a thermocouple in a bore. In $1800 \mathrm{~s}$ microwave pulses, all prototypes reached thermal equilibrium. The equilibrium temperature of each coil is determined by its microwave absorption, its thermal emissivity (both of which are poorly known) and the thermal conductance along the coil, and through the interfaces to the base plate. The heat con- 
ductivities of the tested ceramics are listed in table 3. The lowest temperatures were

\begin{tabular}{|l|c|c|c|c|}
\hline Material & $\mathrm{AlN}$ & $\mathrm{Al}_{2} \mathrm{O}_{3}$ & $\mathrm{BN}$ & $\mathrm{BN}+\mathrm{ZrO}_{2}$ \\
\hline Heat conductivity $[\mathrm{W} /(\mathrm{Km})$ & $90-170$ & $6-30$ & $20-120$ & $30-40$ \\
\hline
\end{tabular}

Table 3: Heat conductivities of the different ceramic materials used for prototype tests of Mirnov coils. The coil formers are usually sintered from powder. The properties of the pieces therefore depend on the procedure followed by the manufacturer. The values in this table are taken from the data sheets of various manufacturers.

observed in the AlN and BN prototypes.

For the final design, AlN was chosen for the coil formers due to its better heat conductivity. The side plates separating the two winding layers are made of $\mathrm{Al}_{2} \mathrm{O}_{3}$ for better machinability. For these side plates, the heat conductance is limited by the interfaces to the coil former at both ends, such that the use of a material with higher heat conductivity would not much improve the overall heat conductance.

To verify the thermal design, a finite element analysis of the temperatures to be expected under stationary and under transient heat loads was performed, taking into account both ECR stray radiation and thermal radiation from the plasma or from the neighbouring WPEs [47]. The analysis demonstrated that the heat conductances across the interfaces between coil former and $\mathrm{CuCrZr}$ bracket and between this bracket and the cooling pipe are important design parameters. This is also true for the heat conductance along the $\mathrm{CuCrZr}$ bracket, which depends on the cross-sectional area and on the length of the bracket.

The highest temperature was found in the $\mathrm{Al}_{2} \mathrm{O}_{3}$ side plates, as expected. Behind graphite tiles loaded with $500 \mathrm{~kW} / \mathrm{m}^{2}$, this temperature reached $\sim 750^{\circ} \mathrm{C}$ under worstcase assumptions on microwave absorption and thermal emissivity, whereas the maximum temperature in the coil former was $\sim 460^{\circ} \mathrm{C}$. Both values are acceptable for the materials used to build the Mirnov coils.

For the winding wire, Constantan ${ }^{\circledR}$ was chosen because of the low temperature dependence of its electric resistivity, such that the resistance change of the coil circuit remains small in spite of the high temperatures expected for the Mirnov coils. The winding wire used is covered by a ceramic insulation layer, although we rather rely on the mere spatial separation of the turns to avoid shorts within the winding. The purpose of the ceramic layer is mainly to avoid shorts in case carbon layers should be deposited on the open windings.

\subsection{Expected signals}

Close to the plasma edge, relative magnetic fluctuation amplitudes $\delta B / B_{0} \sim 10^{-5} \ldots 10^{-3}$ are expected in the $\mathrm{kHz}$ to $\mathrm{MHz}$ range. The largest amplitudes usually occur at low frequencies and low poloidal mode numbers. In a Mirnov coil located $10 \mathrm{~cm}$ outside the plasma edge, the measured magnetic fluctuation amplitude is reduced by a factor $(5 / 6)^{-(m+1)}$ for a poloidal mode number $m$ (see section 2.1.5), based on a radial location of the mode at $r=0.5 \mathrm{~m}$. This reduction factor is $\sim 0.5$ for an $m=3$ mode and $\sim 0.25$ for an $m=6$ mode, where additional damping due to conducting structures between 
plasma and Mirnov coil is disregarded. In the Mirnov coils located behind gaps between wall protection tiles, with their winding area of $\sim 7000 \mathrm{~mm}^{2}$, a $10 \mathrm{kHz} m=1$ mode with $\delta B / B_{0} \sim 10^{-3}$ would induce a signal amplitude of $\pm 1 \mathrm{~V}$.

The minimum relative fluctuation amplitude $\left(\delta B / B_{0}\right)_{\text {min }}$ which can still be measured depends of course on the minimum signal amplitude $U_{\text {min }}$ which can still be distinguished from noise (e.g., by correlation techniques, using the signals of multiple Mirnov coils). For a mode with frequency $f$ and poloidal mode number $m$ located at minor radius $r$, the minimum detectable relative flucutation amplitude is

$$
\left(\delta B / B_{0}\right)_{\min }=\frac{U_{\min }}{110 \mathrm{~V}} \cdot \frac{1 \mathrm{kHz}}{f}\left(\frac{0.6 \mathrm{~m}}{r}\right)^{m+1} .
$$

In contrast to the magnetic equilibrium diagnostics discussed in sections 4-6, the Mirnov coil signals need not to be integrated in time to obtain useful information. Therefore, slowly changing signal components, as they might occur due to thermovoltages or thermal expansion, are of no concern. In contrast, all potential perturbations in the $\mathrm{kHz}$ to $\mathrm{MHz}$ frequency range must be considered. Along most of their length, the signal cables are shielded twisted-pair cables. In some locations, in particular close to the multi-pin connectors used at the vacuum feedthroughs, the shields have to be removed. To avoid cross-talk between the signal cables of different Mirnov coils, additional meshes are employed there to shield the individual pairs from each other.

A brief estimate of the resonance frequencies for mechanical vibration of the Mirnov coils with their $\mathrm{CuCrZr}$ brackets attached to the cooling pipes of the WPEs yields values around $10 \mathrm{kHz}$, well within the frequency range of interest for Mirnov coil signals. Within a realistic gradient of $0.5 \mathrm{~T} / \mathrm{m}$ of the $\mathrm{W} 7-\mathrm{X}$ main magnetic field, vibrations at $10 \mathrm{kHz}$ with an amplitude of $5 \mu \mathrm{m}$ could already generate a signal amplitude of $1 \mathrm{mV}$. The winding wire is not wound around the coil former under significant tension. The restoring force for a length of winding wire is therefore mostly due to its stiffness against bending, and we estimate again resonance frequencies in the range $1-10 \mathrm{kHz}$ for wire lengths of the order of $10 \mathrm{~mm}$. Mechanical vibrations would most probably be excited by turbulence within the cooling pipes, which would be present independent of the plasma. The resulting signal components could therefore be identified and removed during the signal analysis.

We also have to consider fluctuating currents within the plasma vessel as a source of perturbations. If we consider an ELM, it was shown that it consits of filamentary substructures with a size of few centimetres perpendicular to the magnetic field (see, e.g., [48]). In these "blobs", currents of 10-20 A parallel to the magnetic field are expected for a size of $2 \mathrm{~cm} \perp \boldsymbol{B}$. This electric current may continue onto a target plate or WPE and further through its fixation and the PV to close to the plasma through a different location within W7-X. If this location is in a different divertor module, the current may flow toroidally through the PV and generate a poloidal magnetic field component which is recorded by some Mirnov coil. While the fluctuating magnetic field generated by the section of the current path through the plasma should be measured by Mirnov coils, the current path within the PV is considered as perturbation. In order to estimate the amplitude of this perturbation, let us assume that a fluctuating current of $20 \mathrm{~A}$ is distributed over a width $w \sim 50 \mathrm{~cm}$ in the PV. This will generate a magnetic field $(1 / 2) \mu_{0} I / w \sim 25 \mu \mathrm{T}$ close to $(\ll w)$ the PV. The signal induced in a Mirnov coil in this 
location would be $0.1 \mathrm{~V}$ for a frequency of $100 \mathrm{kHz}$, which is a considerable perturbation signal. Although this can be considered a worst case estimate, no simple measures exist to distinguish the signals due to currents in the plasma from those due to currents in the PV and WPEs.

\section{Signal cables}

\subsection{Type of cable}

For the CRCs and SRCs (outside and inside the PV), the DLs and the Mirnov coils (inside the PV), twisted-pair cables with polyimide insulation and a mesh for electromagnetic screening are used. In the vacuum feedthroughs, using multi-pin connectors, each wire of the MED signal cables is fed through two pins, as discussed in section 3.1.2.2 and shown in fig. 3, to reduce thermovoltages. The shielding meshes of the signal cables are insulated at the coil end and are fed through separate pins at the vacuum feedthroughs. They are then connected to the cryostat ground outside the feedthroughs.

The same scheme is used for the signal cables of the Pt1000 sensors integrated in the in-vessel RCs and DLs, except that only one pin per wire is used in the feedthroughs. The Pt1000 sensors are connected with two wires each to limit the amount of cabling (in particular inside the PV). It is therefore necessary to estimate the uncertainty due to temperature changes of the signal cables. The resistance of the Pt1000 sensors changes by $3.9 \Omega / \mathrm{K}$ around $20^{\circ} \mathrm{C}$ and by $3.6 \Omega / \mathrm{K}$ around $250^{\circ} \mathrm{C}$. For the signal cables, the maximum length between Pt1000 sensor and vacuum feedthrough is below $10 \mathrm{~m}$, resulting in a resistance $<4.7 \Omega$, and we assume $150 \mathrm{~m}$ between vacuum feedthrough and cubicle, resulting in additional $90 \Omega$. For the in-vessel section, we assume a maximum temperature of $150^{\circ} \mathrm{C}$ of the signal cables, hence an uncertainty in temperature of $130 \mathrm{~K}$, for the section between vacuum feedthroughs and cubicle we assume a temperature uncertainty of $10 \mathrm{~K}$. With the thermal coefficient of the resistivity of $\mathrm{Cu}$ of $4.33 \cdot 10^{-3} / \mathrm{K}$ (at $0^{\circ} \mathrm{C}$ ), the overall uncertainty of the ohmic resistance of the signal cables will be $<6.5 \Omega$, corresponding to a maximum uncertainty of $1.8 \mathrm{~K}$ in the derived temperature at the sensor location. This uncertainty is tolerable.

For the signal cables of Mirnov coils, special care is taken to avoid cross-talk by grounding the feedthrough pins between the pins of different coils.

\subsection{ECR stray radiation shielding}

Inside the PV, the polyimide-insulated signal cables must be shielded from the ECR stray radiation, like the winding wires of the MEDs. Therefore, the in-vessel signal cables are running in $\mathrm{Cu}$ tubes of $12 \mathrm{~mm}$ or $22 \mathrm{~mm}$ outer diametre with $1 \mathrm{~mm}$ wall thickness. $0.5-$ $0.7 \mathrm{~mm}$ diametre holes are drilled in the walls of the $\mathrm{Cu}$ tubes for pumping ( 1 hole per $100 \mathrm{~mm}$ of tube length).

The heat deposited in the $\mathrm{Cu}$ tubes by absorption of ECR stray radiation and of thermal radiation from the back faces of WPEs must be conducted to the PV. Due to the good thermal conductance along the $\mathrm{Cu}$ tubes, a $\mathrm{Cu}$ bracket bolted to the PV every 
$\sim 30 \mathrm{~cm}$ of tube length is sufficient for this purpose. Due to the low ohmic resistance along these $\mathrm{Cu}$ tubes, the currents induced during a fast decay of the plasma could deform the brackets and the $\mathrm{Cu}$ tubes. Therefore, additional SS brackets are used above the $\mathrm{Cu}$ brackets to reinforce the mechanical connection to the PV.

In several locations, branch connections and connection boxes are used to combine signal cables from different $\mathrm{Cu}$ tubes into a single tube without breaking the ECR stray radiation shielding. Connection boxes are also used to accomodate the crimp connections between the winding wires of the DLs and Mirnov coils and the signal cables. The branch connections and connection boxes were machined from $\mathrm{Cu}$ blocks, after boxes folded from $\mathrm{Cu}$ sheets had shown insufficient ECR radiation shielding in a MISTRAL test, due to remaining gaps. The branch connections are clamped to the connecting $\mathrm{Cu}$ tubes, the connection boxes are bolted to the PV, and additional $\mathrm{Cu}$ brackets ensure good thermal contact between each connection box and the PV.

The in-vessel shielding tubes continue through ports to the feedthroughs, or they open into collector boxes, which are connected to a cable duct through the port (see fig. 30 for an example). By this arrangement, the in-vessel signal cables are running in a space entirely

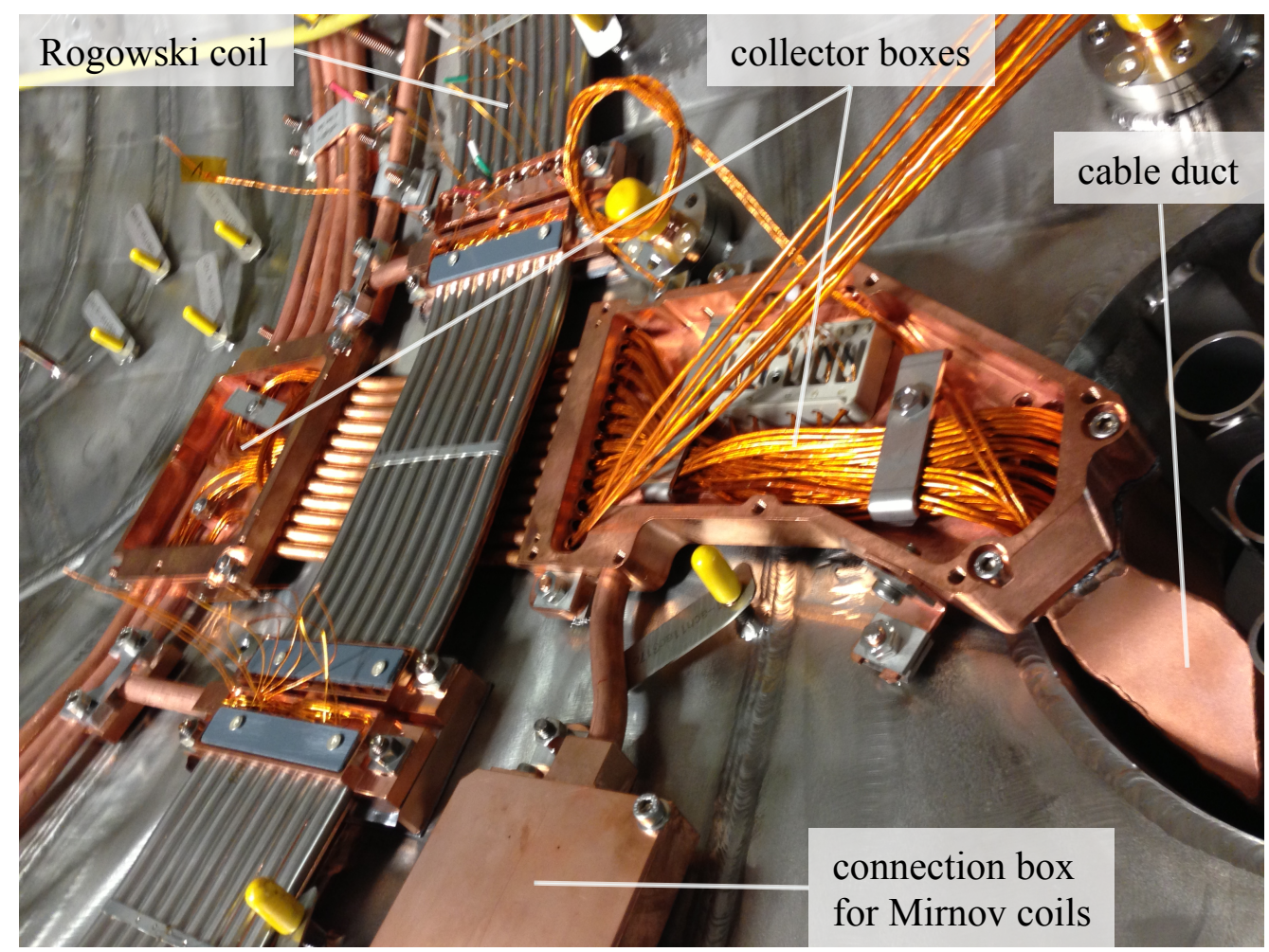

Figure 30: $\mathrm{Cu}$ tubes, connection box for Mirnov coils, collector boxes (still open) and transition into the cable duct through a port for ECR stray radiation shielding of polyimideinsulated signal cables of magnetic diagnostics. In this location, the signal cables are crossing a CRC (SS tubes) in a set of small $\mathrm{Cu}$ tubes due to the very limited space below the WPEs (not yet installed). The connection and collector boxes are machined from $\mathrm{Cu}$ blocks to obtain sufficiently small gaps once their lids are closed.

shielded from the ECR stray radiation, except for pumping holes which are smaller than half the microwave wavelength. 


\subsection{Cabling outside the vacuum barriers}

Outside the multi-pin vacuum feedthroughs, the signal cables of the MEDs continue as individually screened twisted pairs to cubicles with the electronics and data acquisition (see section 9), which are distributed within the W7-X torus hall, such that the length of the signal cables between feedthrough and cubicle is limited to $\sim 20 \mathrm{~m}$.

The signal cables from the Pt1000 sensors, which are distributed in the in-vessel RCs and in the DLs, extend over a larger length and are collected in a single cubicle. Individually screened twisted pairs are used also for these signal cables.

For the Mirnov coils, differential pre-amplifiers will be installed close to the vacuum feedthroughs. The overall length of signal cable between Mirnov coil and pre-amplifier can thus be kept below $10 \mathrm{~m}$, avoiding a high cable capacitance in the Mirnov coil circuits. In the pre-amplifiers, a passive low-pass filter with smooth damping will be implemented for each coil circuit to reduce the amplitude of perturbations in the multi-MHz range, which could occur mainly due to ion cyclotron resonance heating, ICRH. The filter design will take into account both common mode and differentially coupled perturbation signals. The signals will then be transmitted over a length of the order of $100 \mathrm{~m}$ by category- 7 network cables with 4 individually screened twisted pairs to the cubicles with analog-todigital converters, where three pairs will be used for signal transmission and the fourth pair for the power supply of the pre-amplifiers.

\section{Electronics and data acquisition for the magnetic equilibrium diagnostics}

The challenge in long-term integration is, as discussed in section 3.1, to avoid that offset voltages result in a drift of the integrated signal although the magnetic flux through the pick-up coil does not change in reality. One important source of such offset voltages are thermovoltages or thermal drifts in the properties of electronic components. Up to now, analogue electronics were used in most magnetic plasma confinement devices to integrate the signals of the MEDs. In order to correct the signals of a DL for the portion due to changes in the field coil currents, a CC was designed with an appropriate winding area and switched in series with the DL. In W7-X, a separate circuit exists for each winding of a magnetic pick-up coil. Choppers are used at a very early stage of the electronics, which periodically reverse the polarity of the signals with a frequency of $1 \mathrm{kHz}$. In the chopper, semiconductor components are used as switches rather than as resistors, which minimises the effect of thermal drifts on their properties. Any drifts introduced by the electronics after the chopper stage can thus be distinguished from drifts before the chopper stage, since they will not change polarity with the chopper frequency. The chopped signal is then digitised with a sampling rate of $2 \mathrm{MHz}$ and 16 bit resolution (see the discussion in section 3.1.3). The integration itself and also the correction of DL signals by CC signals is then performed numerically. It was demonstrated in [4] that such a solution satisfies the requirements of the W7-X MEDs regarding long-term stability of the integrated signals.

The stages described so far are galvanically isolated and grounded at the vacuum feedthroughs by the screens of their individual signal cables. Up to 32 electronics channels 
of this type are accomodated on an ATCA (Advanced Telecommunications Computing Architecture) board [49]. This board also contains a Field-Programmable Gate Array (FPGA), which can be used for real-time signal analysis. It will then through its own digital output generate a signal for the machine control, e.g., to switch off the plasma heating if the diamagnetic energy is below some minimum value for a certain period of time.

Due to changes in the temperature of the windings and of the in-vessel signal cables, the ohmic resistance of the coil circuits may change. If the input impedance of the electronics is not significantly larger than the resistance of the remaining part of the coil circuit, this must be taken into account to achieve the desired measurement accuracy. Depending on the results during the first operation phase of W7-X, where plasma discharges of more than $\sim 30$ s are not envisaged and the requirements on the measurement accuracy are correspondingly smaller, it may become necessary to further develop the electronics, such that they will be able to monitor the ohmic resistance of the coil circuit during the measurement in the subsequent long-pulse operation phase of W7-X.

\section{Conclusion and outlook}

The major challenges for the design of the W7-X magnetic diagnostics were the reliable long-pulse integration of small signals, the narrow available space for the accomodation of the pick-up coils and, for the diagnostics inside the PV, the potentially high level of ECR stray radiation and the high overall thermal load.

Since the magnetic equilibrium diagnostics rely on the integration of voltages induced in the pick-up coils, any drifts in offset voltages (e. g., thermovoltages), or in the properties of the measurement circuit or of the electronics used to filter, amplify and digitise the signals, can result in large errors in the integrated signals. The portion of this perturbation due to the electronics could be reduced sufficiently by a design using a chopper stage as the electronic front end [4], which reverses the polarity of the input signal periodically. The chopped signal is first digitised and then integrated numerically. In contrast to the approach in other devices, the signals for the compensation of the toroidal magnetic field in the diamagnetic loop signals will be digitised separately. The compensation will then be performed numerically. This approach allowed to optimise the design of the compensation coils for low self-inductance, as a prerequisite for high time resolution.

Perturbations generated outside the electronics cannot, as a matter of principle, be separated from the unperturbed signal by measures within the electronics. Therefore, the effect of thermovoltages in the portion of the measurement circuits outside the electronics, consisting of pick-up coils, signal cables and vacuum feedthroughs, is minimised by avoiding wire connections or by their careful design (see section 3.1.2.2).

The conflicting requirements on the pick-up coils inside the PV of good ECR stray radiation shielding of winding wires and signal cables, good thermal conductance toward the PV, but sufficiently good time resolution, could be reconciled by sophisticated designs with different thermal and electric conductance parallel and perpendicular to the direction of winding, as was demonstrated by the tests described in sections 4.1 and 6.1.

Nevertheless, the measurements in long-pulse discharges remain a challenge due to the 
effects discussed in sections 3.1.3, 3.1.4, 4.2, 5.2 and 6.2. In particular, the potential thermal expansion of the diamagnetic loops may force us to use novel ways to determine the diamagnetic energy on long time scales (see section 4.2). Paths to meet these challenges, as indicated in the corresponding sections, will have to be explored in more detail in the shorter discharges during the initial operation phases of W7-X [10].

The installation of components of the magnetic diagnostics in the cryovacuum and inside the plasma vessel has now been completed (except for those components not to be installed before the first operation phase of W7-X). Presently, the signal cables outside the vacuum feedthroughs and the electronics and ADCs are under preparation.

For a correct analysis of the magnetic equilibrium diagnostic signals it is necessary to take into account the coupling between the field coil circuits and the diagnostic coil circuits. This coupling can, in principle, be calculated from the coil geometries. The technical commissioning of the field coil systems will provide the opportunity for an experimental verification. This will serve as a first part of the calibration of the magnetic diagnostics.

The second part of the calibration will use exciter coils inside the plasma vessel, which will allow to assess the response of the diagnostic coils to currents in the plasma volume, including the effects of eddy currents in the plasma vessel and in the various in-vessel components. In order to fully profit from this part of the calibration, the position of the exciter coils within the plasma vessel will have to be surveyed precisely. This second part of the calibration is therefore planned during a maintenance interval after the first phase of W7-X plasma operation.

\begin{tabular}{lll}
\hline Abbreviation & Meaning & $\begin{array}{l}\text { Introduced } \\
\text { in section }\end{array}$ \\
\hline BP & bean-shaped plane & 2.1 .1 \\
CC & compensation coil & 2.1 .2 \\
CRC & continuous Rogowski coil & 2.1 .1 \\
DL & diamagnetic loop & 1 \\
HM & half module & 2.1 .1 \\
MED & magnetic equilibrium diagnostic & 1 \\
MISTRAL & MIcrowave STray RAdiation Launch facility & 3.2 \\
MPTS & magnetic probe test stand & 3.2 \\
PS current & Pfirsch-Schlüter current & 1 \\
PV & plasma vessel & 1 \\
RC & Rogowski coil & 1 \\
SC & saddle coil & 1 \\
SRC & segmented Rogowski coil & 2.1 .1 \\
TP & triangular plane & 2.1 .1 \\
WPE & wall protection element & 2.1 .1 \\
\hline
\end{tabular}

Table 4: List of abbreviations not in common use. 


\section{Acknowledgements}

In addition to the authors, some 50 further colleagues at the IPP Greifswald and Garching sites contributed to the realisation of the W7-X magnetic diagnostics by organising the supply of raw materials and components, by machining and assembling them, by integrating the coils, pipework and cabling both into the work schedules, and then physically into W7-X, by surveying, winding coils, qualifying and executing crimp connections, by checking the quality, and by providing time schedule and financial coordination. A part of the manufacturing was also performed at external companies. Without the accurate work of all these people it would not have been possible to build these complex diagnostics and to integrate them into the W7-X device.

We especially thank R. Binder, T. Broszat and J. Ernst of the W7-X design office for the inventive design solutions they developped and for the thorough work placing our diagnostics into the narrow space between other components.

We also acknowledge valuable discussions on ECR stray radiation with M. Hirsch and H. P. Laqua, and we thank M. Gasparotto, R. König, T. S. Pedersen, F. Wagner and R. Wolf for their continuing interest and support.

\section{References}

[1] C. Beidler et al., Fusion Technology 17, 148 (1990).

[2] H.-S. Bosch et al., Nucl. Fusion 53, 126001 (2013).

[3] H.-J. Hartfuß, R. König, and A. Werner, Plasma Phys. Controlled Fusion 48, R83 (2006), Topical Review.

[4] A. Werner, Rev. Sci. Instrum. 77, 10E307 (2006), Proceedings of the 16th Topical Conference on High-Temperature Plasma Diagnostics, Williamsburg, VA, USA, 7-11 May 2006.

[5] W. Lotz, J. Nührenberg, and C. Schwab, Optimization, MHD mode and alpha particle confinement behaviour of HELIAS equilibria, in Proceedings of the 13th Conference on Plasma Physics and Controlled Nuclear Fusion Research, Washington 1990, volume 2, pages 603-611, Wien, 1991, International Atomic Energy Agency, IAEA, paper IAEA-CN-53/C-III-5.

[6] I. H. Hutchinson, Principles of plasma diagnostics, Cambridge University Press, Cambridge, New York, Port Chester, Melbourne, Sydney, 1987.

[7] J. Geiger, M. Endler, and A. Werner, Contrib. Plasma Phys. 50, 736 (2010), Special Issue: Proceedings of 17th International Stellarator/Heliotron Workshop, October 12-16, 2009, Princeton Plasma Physics Laboratory (PPPL), Princeton, New Jersey, USA (Part II).

[8] P. Moreau et al., Fusion Eng. Design 86, 1222 (2011), Proceedings of the 26th Symposium on Fusion Technology, Porto, Portugal, 27 September-1 October 2010. 
[9] G. Grieger et al., Phys. Fluids B 4, 2081 (1992).

[10] H.-S. Bosch et al., IEEE Trans. Plasma Sci. 42, 432 (2014).

[11] S. Besshou, C. E. Thomas, T. Ohba, A. Iiyoshi, and K. Uo, Nucl. Fusion 26, 1339 (1986).

[12] V. D. Pustovitov, J. Plasma Fusion Res. 69, 34 (1993).

[13] W. A. Cooper and A. J. Wootton, Plasma Phys. 24, 1183 (1982).

[14] S. Besshou et al., Rev. Sci. Instrum. 72, 3859 (2001).

[15] H. Zohm, Plasma Phys. Controlled Fusion 38, 105 (1996), http://www.iop.org/EJ/ article/0741-3335/38/2/001/p602r1.pdf.

[16] ASDEX Team, Nucl. Fusion 29, 1959 (1989).

[17] I. Nunes et al., Study of ELMs on ASDEX Upgrade using reflectometry measurements with high temporal and spatial resolution, in 29th EPS Conference on Plasma Physics and Controlled Fusion (Montreux), edited by R. Behn and C. Varandas, volume 26B, European Physical Society, 2002, paper O-5.06, http://epsppd.epfl.ch/Montreux/ pdf/O5_06.pdf.

[18] V. D. Shafranov: "Plasma Equilibrium in a magnetic field" in Reviews of Plasma Physics 2 (1966), M. A. Leontovich, editor, pages 103-151.

[19] T. Yamaguchi et al., Nucl. Fusion 45, L33 (2005).

[20] Y. Asahi, Y. Suzuki, K. Y. Watanabe, and W. A. Cooper, Phys. Plasmas 20, 022503 (2013).

[21] N. B. Marushchenko et al., EPJ Web of Conferences 32, 01004 (2012), http:// dx.doi.org/10.1051/epjconf/20123201004.

[22] C. Nührenberg, Alfvén eigenmodes in Wendelstein 7-X - equilibrium properties and spectral gap structure, in Proceedings of the Joint Varenna-Lausanne International Workshop on Theory of Fusion Plasmas (ISPP-19, 28 August-1 September 2000, Varenna), edited by J. W. Connor, O. Sauter, and E. Sindoni, page 313, Bologna, 2000, Società Italiana di Fisica.

[23] S. Zegenhagen, A. Werner, A. Weller, and T. Klinger, Plasma Phys. Controlled Fusion 48, 1333 (2006).

[24] M. Nagel, S. Y. Shim, and F. Schauer, Fusion Eng. Design 75-79, 139 (2005), Proceedings of the 23rd Symposium on Fusion Technology, Venice, Italy, 20-24 September 2004. 
[25] M. Nagel et al., Design and assembly progress of the thermal insulation of the Wendelstein 7-X cryostat, in Proceedings of the 23rd International Cryogenic Engineering Conference and International Cryogenic Materials Conference 2010, ICEC23-ICMC 2010, Wroclaw, Poland, pages 815-820, 2010.

[26] J. Boscary et al., Fusion Eng. Design 86, 572 (2011), Proceedings of the 26th Symposium on Fusion Technology (SOFT-26), Porto, Portugal, September 27-October 1, 2010.

[27] B. Mendelevitch et al., Fusion Eng. Design 88, 1660 (2013), Proceedings of the 27th Symposium on Fusion Technology (SOFT-27), Liège, Belgium, September 24-28, 2012.

[28] A. Peacock et al., Fusion Eng. Design 86, 1706 (2011), Proceedings of the 26th Symposium on Fusion Technology (SOFT-26), Porto, Portugal, September 27-October 1, 2010.

[29] F. Schauer, E. Harmeyer, and B. Hein, Wirbelstromkräfte auf das W7-X-Plasmagefäß durch Plasmastromabbrüche, Technical report, Max-Planck-Institut für Plasmaphysik, Greifswald, 2009.

[30] F. Gadelmeier et al., Conditions for island divertor operation in the W7-AS stellarator, in 28th EPS Conference on Controlled Fusion and Plasma Physics (Funchal, Madeira), edited by C. Silva, C. Varandas, and D. Campbell, volume 25A, pages 2085-2088, European Physical Society, 2001, Poster P5.096, http:// www.cfn.ist.utl.pt/EPS2001/fin/pdf/P5.096.pdf.

[31] T. Sunn Pedersen et al., Plans for first physics operation of Wendelstein 7-X, 2015, in preparation.

[32] V. Erckmann et al., Fusion Sci. Tech. 52, 291 (2007).

[33] D. Hathiramani et al., Fusion Eng. Design 88, 1232 (2013), Proceedings of the 27th Symposium on Fusion Technology (SOFT-27), Liège, Belgium, 24-28 September 2012 .

[34] J. W. Lamb, International Journal of Infrared and Millimeter Waves 17, 1997 (1996).

[35] W. Kasparek, A. Fernandez, F. Hollmann, and R. Wacker, International Journal of Infrared and Millimeter Waves 22, 1695 (2001).

[36] H. Kumrić, E. Häberle, and W. Kasparek, Characterization of surface losses of plasma-facing mirror samples, Technical report, Institut für Plasmaforschung, Universität Stuttgart, 2008, ITER task "Design, analysis and testing of the upper launcher and port plug for the ITER ECH\&CD system", subtask "FS mm-wave components test", final report for deliverable b.7.1.

[37] M. Hirsch et al., The impact of microwave stray radiation to in-vessel diagnostic components, in Proceedings of the International Conference on Fusion Reactor Diagnostics (9-13 September, 2013, Varenna), 2013. 
[38] R. Burhenn et al., Contrib. Plasma Phys. 51, 271 (2011), Proceedings of Plasma Diagnostics 2010, 12-16 April 2010, Pont-à-Mousson, France.

[39] http://www.stl-gmbh.de.

[40] A. Carls, M. Köppen, J. Fellinger, and F. Schauer, Fusion Eng. Design 88, 1433 (2013), Proceedings of the 27th Symposium on Fusion Technology (SOFT-27), Liège, Belgium, 24-28 September 2012.

[41] X. B. Peng et al., Fusion Eng. Design 88, 1727 (2013), Proceedings of the 27th Symposium on Fusion Technology (SOFT-27), Liège, Belgium, 24-28 September 2012.

[42] H. Greuner et al., Fusion Eng. Design 66-68, 447 (2003), Proceedings of the 22nd Symposium on Fusion Technology, Helsinki, Finland, 9-13 September 2002.

[43] http://www.lampert.info/en.

[44] http://www.keramikblech.com.

[45] P. Thomas, Diamagnetic flux measurement using the PDX TF coils, Technical Report PPPL-1979, Princeton Plasma Physics Laboratory, 1983.

[46] A. Werner, M. Endler, J. Geiger, and R. König, Rev. Sci. Instrum. 79, 10F122 (2008), Proceedings of the 17th Topical Conference on High-Temperature Plasma Diagnostics, Albuquerque, New Mexico, USA, 11-15 May 2008.

[47] M. Y. Ye, X. B. Peng, L. Fransico, and M. Endler, Fusion Eng. Design 88, 1844 (2013), Proceedings of the 27th Symposium on Fusion Technology (SOFT-27), Liège, Belgium, 24-28 September 2012.

[48] M. Endler et al., Plasma Phys. Controlled Fusion 47, 219 (2005), http:// stacks.iop.org/0741-3335/47/219.

[49] B. Gonçalves et al., IEEE Trans. Nucl. Sci. 57, 2147 (2010). 